\title{
PHASE COEXISTENCE IN ISING, POTTS AND PERCOLATION MODELS क
}

\author{
Raphaël CERF ${ }^{\mathrm{a}}$, Ágoston PISZTORA ${ }^{\mathrm{b}}$ \\ ${ }^{a}$ CNRS, Université Paris Sud, Mathématique, Bâtiment 425, 91405 Orsay Cedex, France \\ ${ }^{\mathrm{b}}$ Department of Mathematical Sciences, Carnegie Mellon University, Pittsburgh, PA 15213, USA
}

Received 13 March 2000, revised 27 February 2001

ABSTRACT. - We study phase coexistence (separation) phenomena in Ising, Potts and random cluster models in dimensions $d \geqslant 3$ below the critical temperature. The simultaneous occurrence of several phases is typical for systems with appropriately arranged (mixed) boundary conditions or for systems satisfying certain physically natural constraints (canonical ensembles). The various phases emerging in these models define a partition, called the empirical phase partition, of the space. Our main results are large deviations principles for (the shape of) the empirical phase partition. More specifically, we establish a general large deviation principle for the partition induced by large (macroscopic) clusters in the Fortuin-Kasteleyn model and transfer it to the Ising-Potts model where we obtain a large deviation principle for the empirical phase partition induced by the various phases. The rate function turns out to be the total surface free energy (associated with the surface tension of the model and with boundary conditions) which can be naturally assigned to each reasonable partition. These LDP-s imply a weak law of large numbers: asymptotically, the law of the phase partition is determined by an appropriate variational problem. More precisely, the empirical phase partition will be close to some partition which is compatible with the constraints imposed on the system and which minimizes the total surface free energy. A general compactness argument guarantees the existence of at least one such minimizing partition. Our results are valid for temperatures $T$ below a limit of slab-thresholds $\widehat{T}_{c}$ conjectured to agree with the critical point $T_{c}$. Moreover, $T$ should be such that there exists only one translation invariant infinite volume state in the corresponding Fortuin-Kasteleyn model; a property which can fail for at most countably many values and which is conjectured to be true for every $T \neq T_{c}$. ๑ 2001 Éditions scientifiques et médicales Elsevier SAS

Keywords: Ising model; Potts model; Random cluster model; FK percolation; Phase separation; Phase coexistence; Large deviations; Double bubble; Minimal surfaces; Caccioppoli partitions

AMS classification: $60 \mathrm{~K} 35 ; 82 \mathrm{Bxx} ; 60 \mathrm{~F} 10$

RÉSUMÉ. - Nous étudions le phénomène de coexistence (et de séparation) des phases dans les modèles d'Ising, de Potts et de clusters aléatoires en dimension $d \geqslant 3$ en-dessous de la

\footnotetext{
The authors thank the following institutions for their hospitality and financial support: Center for Nonlinear Analysis at CMU, Forschungsinstitut at ETHZ, Université Paris Sud, Orsay. A. Pisztora's research has been supported by NSF Grant DMS-9704901.

E-mail addresses: Raphael.Cerf@math.u-psud.fr (R. Cerf), pisztora@asd4.math.cmu.edu (Á. Pisztora).
} 
température critique. La présence simultanée de plusieurs phases est typique des systèmes avec des conditions au bord mixtes choisies de manière adéquate ou bien des systèmes soumis à des contraintes physiques naturelles (ensembles canoniques). Les diverses phases qui émergent dans ces modèles définissent une partition de l'espace, appelée la partition des phases empirique. Nos résultats principaux sont des principes de grande déviation pour la partition des phases empirique. Plus spécifiquement, nous établissons un principe de grande déviation général pour la partition induite par les grands clusters (macroscopiques) du modèle de Fortuin-Kasteleyn et nous le transférons aux modèles d'Ising et de Potts, dans lesquels nous obtenons un principe de grande déviation pour la partition des phases empirique induite par les différentes phases. La fonction de taux est l'énergie libre de surface totale (associée à la tension de surface du modèle et aux conditions au bord) qui est naturellement assignée à chaque partition raisonnable. Ces PGDs entraînent une loi faible des grands nombres : asymptotiquement, la loi de la partition des phases est déterminée par un problème variationnel adéquat. Plus précisément, la partition des phases sera proche d'une partition compatible avec les contraintes imposées au système et qui minimise l'énergie libre de surface totale. Nos résultats sont valides pour des températures $T$ en-dessous de la limite des points critiques dans les tranches $\widehat{T}_{c}$ qui est conjecturée coïncider avec le point critique $T_{c}$. De plus, $T$ doit être telle qu'il existe seulement une mesure en volume infini invariante par translation dans le modèle de Fortuin-Kasteleyn associé ; une propriété qui peut être violée sur un nombre au plus dénombrable de valeurs et qui est conjecturée être vraie pour toute température $T \neq T_{c}$. $\odot 2001$ Éditions scientifiques et médicales Elsevier SAS

\section{Introduction and main results}

\subsection{Introduction}

In this article we continue the analysis of phase separation and phase coexistence phenomena in the context of Ising-Potts and percolation models in dimensions $d \geqslant 3$, by extending the techniques used in our previous work [15]. The main goal of the present work is to justify, starting from a microscopic point of view, the validity of the basic assumptions underlying the classical phenomenological theory of coexisting phases, namely, that the shapes of coexisting phases are governed by a variational (minimal action) principle. Whereas our previous work [15] focused exclusively on the Wulff problem - a prominent but specific example of phase coexistence - here we will study this phenomenon from a more general point of view. Results and ideas which are relevant or closely related to those contained in the present paper appeared in $[3,4,9,10,12-15,22$, 33-36,39,46,49,51]. Let us notice that several questions handled in the present work have been adressed in the context of two dimensional models [1,47,48,52]. For a summary of the development of this field, in particular for general references and historical remarks we refer the reader to the introduction of [15].

The $q$-states Potts model (a brief description of which is given in Section 2.2) is a natural choice for our purpose since it is probably the simplest example of a Gibbsmeasure exhibiting several "pure" phases, ${ }^{1}$ which can be forced to coexist by imposing appropriate constraints on the system, such as mixed boundary conditions or constraints

\footnotetext{
${ }^{1}$ We use the word "pure" for the phases obtained in the thermodynamic limit by imposing constant boundary conditions.
} 

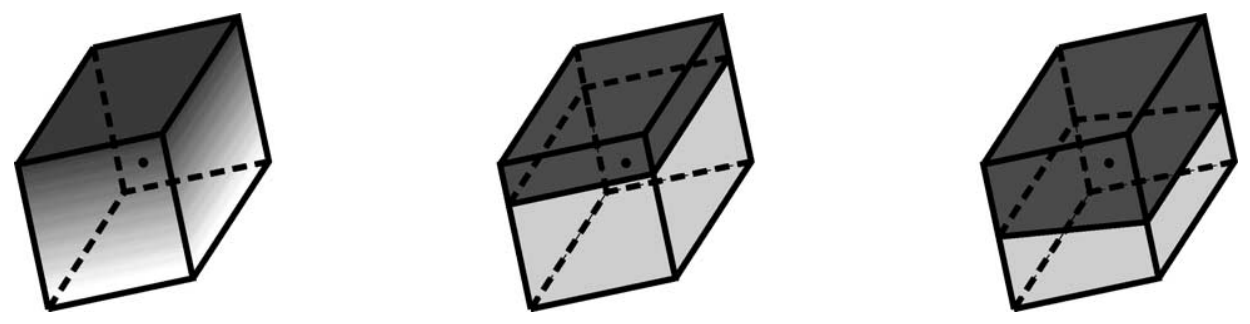

Fig. 1.

on the number of spins in a certain state. Recall that this latter constraint occurs naturally in the lattice gas interpretation of Ising-Potts models: In this case it is simply a constraint on the number of particles of a given type. It seems to be the case that when systems corresponding to discrete spins are submitted to certain conflicting constraints, they exhibit only pure phases, several of which might coexist so as to satisfy the imposed constraints. In particular, the pure phases are separated by sharp (when viewed from the macroscopic perspective) phase boundaries rather than by some wide transition regime where a smooth change between the phases could take place. We will refer to this phenomenon as sharp phase separation into pure phases.

Two fundamental problems arise in this context. The first one is to understand the reason behind the absence of "transitional" states, in particular, the sharpness of phase boundaries. The second problem is to understand the geometry of the emerging phases and to recover the law governing the shapes of the interfaces.

As an example, let us consider the Ising model below the critical temperature in a lattice box which is slightly tilted (in a small angle $\gamma$ ) with respect to the lattice axis. We impose boundary conditions as follows: plus on the top face, minus on the bottom and free on the remaining lateral sides. How does a typical configuration look like? Possible answers are depicted in Fig. 1. According to the leftmost picture there is a continuous transition from plus spins on the top face to minus spins on the bottom. In the middle picture we observe a flat interface parallel to the top and bottom faces separating two regions filled with the pure plus and minus phases, respectively. The rightmost picture is similar to the second one but the interface is not parallel to the top and bottom faces. The angle between the axis direction and the interface is $\alpha$, with $0 \leqslant \alpha \leqslant \gamma$.

We will prove (Corollary 1.5) that in the Ising model with mixed boundary conditions there is indeed sharp phase separation into pure phases, which rules out the first scenario. $^{2}$ Phases will thereby be identified by looking at the value of the local magnetization averaged on an intermediate scale. Once we know that only pure phases, separated by sharp phase boundaries, occur, it is reasonable to focus our attention on the (free) energy penalty created by these interfaces. Indeed, in the phenomenological description one assumes the existence of a direction-dependent macroscopic quantity, called the surface tension $\tau(v)$, such that any piece of an interface between two pure phases carries an energy whose value is equal to the surface integral of $\tau(v)$ over that part of the interface, where $v$ is the unit vector normal to the interface. The fundamental assumption underlying the phenomenological theory of coexisting phases is that in

\footnotetext{
${ }^{2}$ Somewhat surprisingly, no short and convincing heuristic argument seems to be known.
} 
equilibrium, the various phases coexist in such a way that the total energy associated with the interfaces is minimal among all possible phase configurations (partitions) which are compatible with the constraints (for instance boundary conditions or volume constraints) imposed on the system. The goal of the current work is a rigorous derivation of the phenomenological picture described above.

In order to derive a law of large numbers, i.e., to describe the typical behavior of the system, we perform a general large deviation analysis. We point out that the large deviation approach is currently the only known way to achieve this kind of results in dimensions $d \geqslant 3$. Namely, one essential ingredient of the proof is a compactness argument which, similarly as in $[10,12,14,15]$, replaces a combinatorial bound on the entropy. Large deviations, on the other hand, link the microscopic model with the calculus of variations. We have then to provide an adequate framework for the precise formulation of the emerging variational problems. The relevant objects are phase partitions for the spin models and Caccioppoli partitions of finite perimeter for the FK model. We can naturally define a surface energy functional on these objects (which turns out to be a good rate function in the large deviations context). In our analysis we strongly rely on the Fortuin-Kasteleyn random cluster representation of the spin models. The basic results are derived in the FK-percolation setting and in a second step they are transferred to the spin models.

Our main tool, Theorem 1.8, is a general large deviation principle (LDP) for the macroscopic configuration observed in FK percolation. In order to identify the phases on the spin level, we consider the partition of the underlying region associated with large, in fact macroscopic, clusters of the percolation process. In the next step, we obtain on the spin level a LDP for the empirical phase partition corresponding to the different phases visible on the macroscopic scale. We remark that the relevance of continuous partitions to study interfaces has already been outlined in the phenomenological theory of phases coexistence, in particular results related to lower-semicontinuity of functionals on partitions and their $\Gamma$-convergence can be found in $[5,6]$. The LDP-s ensure a weak law of large numbers: the law of the empirical phase partition is determined asymptotically by an appropriate variational problem. With very large probability, the phase partition will be close to a partition whose total surface free energy is minimal under certain requirements corresponding, for example, to boundary conditions or volume constraints. A general compactness argument implies the existence of at least one such minimizer. However, in most examples one cannot say much about the minimizers themselves. (One notable exception is the Wulff problem.) The difficulty stems from the fact that the surface tension $\tau$ is anisotropic and almost no quantitative information about its magnitude is available. Moreover, the corresponding variational problems are extremely hard even in the isotropic case and the (few) resolved questions represent the state of the art in the calculus of variations. For instance, a famous conjecture related to the symmetric double-bubble in the three dimensional case with isotropic surface energy (perimeter) has only been resolved recently [32] and the asymmetric case remains unresolved (even in the isotropic case). In general, results on the regularity of $\tau$ would yield results on the local regularity of minimizing configurations (see [7]). For general results on minimal partitions in the isotropic case, see $[2,40]$. 
Returning to our first example, in order to predict the "typical" empirical phase partition we have to find the ones which minimize the surface free energy. Note that along the lateral walls (where free boundary conditions are imposed) there is no energy penalty, hence no contribution to the surface energy. Let us assume that the sharp triangle inequality holds (see [23]), which implies that the interface $\Phi$ separating the two pure phases is flat. Its tilt will be determined so as to minimize the energy, which is given as the product of the surface area of $\Phi$ and $\tau(\vec{n})$, where $\vec{n}$ is the surface normal. Whether the second or the third picture is "correct" depends on the unknown anisotropy of the surface tension. There is an additional issue worth discussing, namely, the position of the interface $\Phi$. From our analysis it follows that the empirical phase partition will be close to some minimizer. Here, there is a continuum of minima corresponding to any flat interface with the correct tilt but in an arbitrary height. We believe that in the limit $N \rightarrow \infty$, where $N$ denotes the box size, the distribution of the height of the interface is indeed uniform. This has to be contrasted with the finite $N$ case, where presumably more subtle stochastic effects, such as interface fluctuations, should be taken into account to understand the law of the height of the interface. It is natural to conjecture that in this case the height-density is nearly flat but decays rapidly near the top and bottom of the box.

More interesting and complex questions appear naturally in the context of the Potts model with several states. Consider for instance the Potts model with $q=6$ colors (states) in a three dimensional box with boundary condition $i$ on the $i$ th face of the box. Naively, one might expect that all phases will try to occupy the region closest to the corresponding piece of the boundary, which would lead to a phase partition consisting of symmetric and pyramid-like regions, as can be seen in Fig. 2, left. However, at least in the case when the surface tension is isotropic (which is presumably the case in the limit $T \uparrow T_{c}$ ), there exists a better configuration with lower total surface free energy. Recall that in this case our desired interface is simply a minimal surface spanned by the edges of the box. A picture of the well known solution to this problem can be seen in Fig. 2, right. In order to be able to discuss this example at temperatures $0<T<T_{c}$, we have to
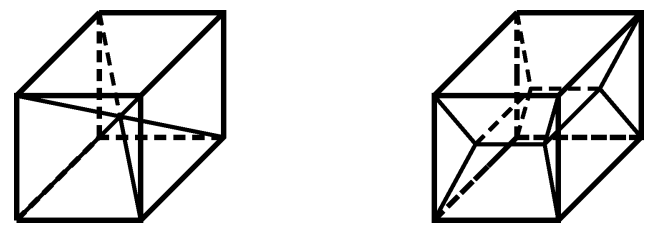

Fig. 2.
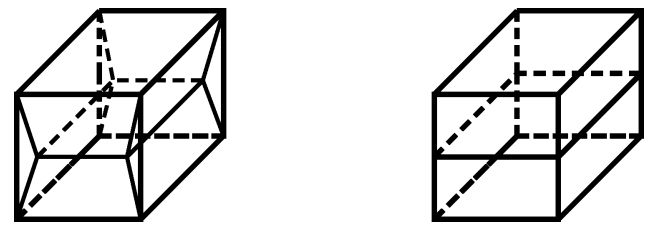

Fig. 3. 
make certain assumptions about the surface tension $\tau$. We assume that the sharp simplex inequality holds, that the value of $\tau$ is minimal in axis directions and that $\tau$ increases as the normal vector moves from say $(0,0,1)$ to $(1,1,1)$. (Although these assumptions are very plausible, none of them has been proved in dimensions $d \geqslant 3$ ). Under these hypotheses, we conjecture that the phase partition at moderate subcritical temperatures looks like in Fig. 3, left. In the limit $T \downarrow 0$, only two phases survive, as shown in Fig. 3, right. At $T=0$, there is no reason for the middle plane to stay centered, in fact, any horizontal plane is equally likely.

In the next example we consider the three dimensional Ising model with free boundary conditions below $T_{c}$, conditioned on the event that the average magnetization is positive and does not exceed $m^{*}-\varepsilon$, where $\varepsilon$ is a sufficiently small positive number and $m^{*}$ denotes the spontaneous magnetization. It is natural to conjecture that the minimizers of the corresponding variational problem look like the picture in Fig. 4. A single bubble sitting in one of the corners is filled with the minus phase and in the rest of the box we see the plus phase. The size of the bubble is determined by $\varepsilon$ and its internal boundary coincides with the corresponding piece of the surface of the Wulff crystal.

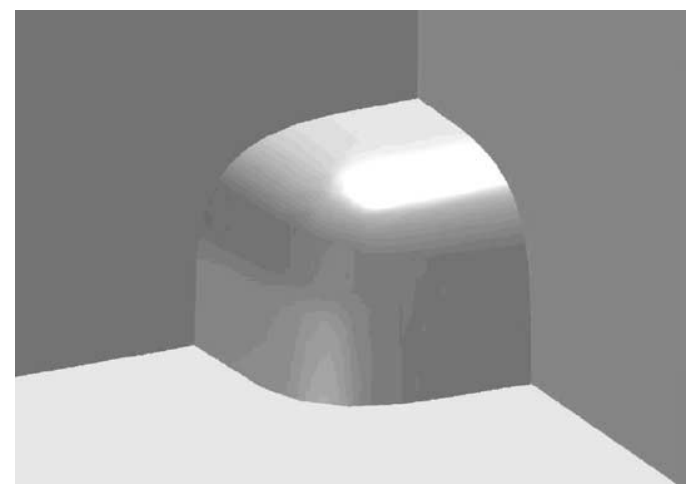

Fig. 4.

Another Wulff-type problem arises by conditioning the $q$-states Potts model (with say $q \geqslant 4$ ) to have a moderate excess of colors 2 and 3 while imposing 1-boundary conditions on the entire box. In this case it is conceivable that a so-called "double

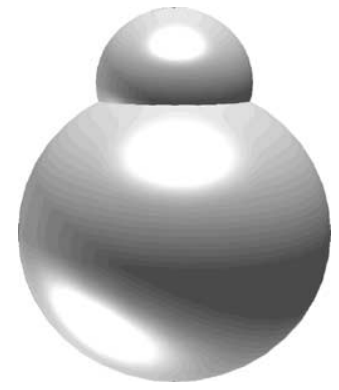

Fig. 5. 


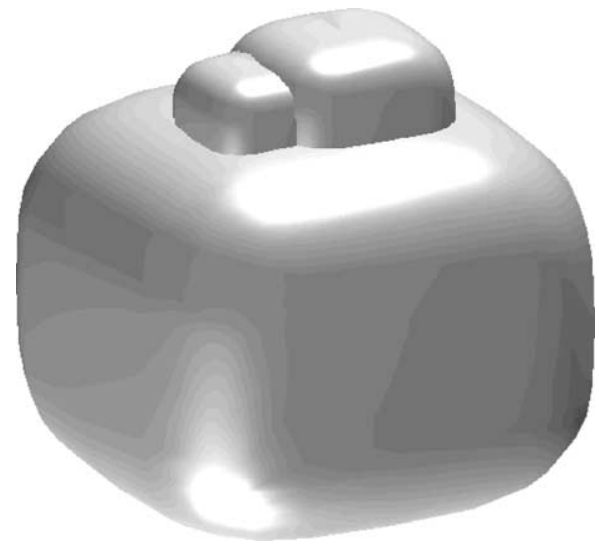

Fig. 6.

bubble" is created, consisting of two adjacent macroscopic droplets filled with the (pure) phases 2 and 3, respectively. The double bubble is swimming in the phase 1 which fills the rest of the box. Fig. 5 shows the double bubble when the surface tension is close to isotropic. Of course, we might have an excess of color 4 as well; in this case a further bubble will presumably appear which will be attached to the previous two bubbles. A picture of such a situation is shown in Fig. 6, where we assumed a relatively strong anisotropy. (We warn the readers that these pictures are guesses and have not been obtained by simulations.)

For related variational questions concerning soap films and immiscible fluids, see [38].

In fact, by studying questions concerning phase boundaries we are very quickly confronted with the theory of minimal surfaces, such as the Plateau problem, corresponding to anisotropic surface measures. Let $\Omega$ be a bounded open set in $\mathbb{R}^{3}$ with smooth boundary and let $\gamma$ be a Jordan curve drawn on $\partial \Omega$ which separates $\partial \Omega$ into two disjoint relatively open sets $\Gamma^{+}$and $\Gamma^{-}$. Typical configurations in the Ising model on a fine grid in $\Omega$ with plus b.c.s on $\Gamma^{+}$and minus b.c.s on $\Gamma^{-}$will exhibit two phases separated with an interface close to a minimal surface which is a global solution of the following Plateau type problem:

$$
\text { minimize } \int_{S} \tau\left(v_{S}(x)\right) \mathrm{d} \mathcal{H}^{d-1}(x): S \text { is a surface in } \Omega \text { spanned by } \gamma,
$$

where $v_{S}(x)$ is the normal vector to $S$ at $x$. We remark that it is conjectured that, as the temperature approaches $T_{c}$ from below, the surface tension $\tau$ becomes more and more isotropic and it is conceivable that the solution of the above minimization problem approaches the solution of the classical (isotropic) Plateau problem.

\subsection{The main results}

We will study Ising-Potts models and FK percolation on certain finite regions of the lattice. We refer to Section 2.2 for the definitions, notation and a brief summary of these models. We consider first FK percolation in dimensions $d \geqslant 3$ in the regime 
$q \geqslant 1, p>\widehat{p}_{c}$ such that $\theta^{f}(p)=\theta^{w}(p)$. Here $\widehat{p}_{c}$ is the slab percolation threshold introduced in [49] which is conjectured to coincide with the critical point $p_{c}$. (This is the case at least for Bernoulli percolation $(q=1)$ by the result of Grimmett and Marstrand [31].) The quantities $\theta^{f}(p), \theta^{w}(p)$ are the densities of the infinite open cluster for the infinite volume FK measures $\Phi_{\infty}^{p, q, f}, \Phi_{\infty}^{p, q, w}$ with free and wired boundary conditions, respectively. The equality $\theta^{f}(p)=\theta^{w}(p)$ implies that there exists a unique infinite volume FK measure $\Phi_{\infty}^{p, q}$ on the cubic lattice $\mathbb{L}^{d}$ (and the converse implication is true as well). It is conjectured that $\theta^{f}(p)=\theta^{w}(p)$ for every $p \neq p_{c}$ and it is known that this is true for values of $p$ close enough to 1 and might be violated for at most countably many values of $p$, cf. [30].

Our approach is based on the Fortuin-Kasteleyn (FK) representation of the IsingPotts model which recovers the Potts measure on the spin level through an independent coloring (with spins) of the clusters of the FK percolation process (see Section 2.2 for a detailed description). The inverse temperature $\beta=1 / T$ in the spin model is related to $p$ via the relation $p=1-\exp (-\beta)$. The parameter $q$ of the FK process is equal to the number of states in the Potts model $\left(q=2\right.$ in the Ising case). We set $\widehat{\beta}_{c}=-\log \left(1-\widehat{p}_{c}\right)$ and $\mathcal{U}(q, d)=\left\{-\ln (1-p): p\right.$ such that $\left.\theta^{f}(p, q)=\theta^{w}(p, q)\right\}$.

Range of validity of the results. Our results for the Ising-Potts models hold in the region: $d \geqslant 3, q \in \mathbb{N} \backslash\{0,1\}, \beta>\widehat{\beta}_{c}(q, d), \beta \in \mathcal{U}(q, d)$. For the FK process, our results hold in the region: $d \geqslant 3, q \geqslant 1, p>\widehat{p}_{c}(q, d)$ such that $\theta^{f}(p, q)=\theta^{w}(p, q)$.

At this point it is natural to discuss the case of two dimensions. Although most of our results should hold for $d=2$, there are several points in the proofs which would require a significant change, making the proofs even longer. The main reason, however, for not to treat the two dimensional case is that the natural topology for the LDP-s in $d=2$ is not the one we use (which is based on the distance dist $_{L^{1}}$ ) but a topology based on the Hausdorff distance. For reasons of space, we refrain from carrying out that analysis here, in fact, that would require a separate publication.

Surface tension. From FK percolation we can extract a direction dependent surface tension $\tau(v)=\tau(p, q, d, v)$, cf. [15]. For a unit vector $v$, let $A$ be a unit hypersquare orthogonal to $v$, let cyl $A$ be the cylinder $A+\mathbb{R} v$, then $\tau(v)$ is equal to the limit

$\lim _{n \rightarrow \infty}-\frac{1}{n^{d-1}} \log \Phi_{\infty}^{p, q}\left(\begin{array}{l}\text { inside } n \text { cyl } A \text { there exists a finite set of closed edges } E \text { cutting } \\ n \text { cyl } A \text { in at least } 2 \text { unbounded components and the edges of } \\ E \text { at distance less than } 2 d \text { from the boundary of } n \text { cyl } A \text { are at } \\ \text { distance less than } 2 d \text { from } n A\end{array}\right)$.

The function $\tau$ satisfies the weak simplex inequality, is continuous, positive and invariant under the isometries which leave $\mathbb{Z}^{d}$ invariant (see Section 4 in [15] for details).

Consider a bounded open region $\Omega$ in $\mathbb{R}^{d}$ with boundary $\Gamma$ satisfying the following hypothesis:

Hypothesis on $\Omega$. - We suppose that $\Omega$ is a Lipschitz domain, i.e., its boundary $\Gamma$ can be locally represented as the graph of a Lipschitz function defined on some open ball of $\mathbb{R}^{d-1}$. 
Note that this hypothesis is automatically satisfied when $\Omega$ is a bounded open set with a $C^{1}$ boundary or when $\Omega$ is a polyhedral domain.

We will study Potts models and FK percolation on the region $\Omega$. To obtain a discretized version of the region $\Omega$, we define for $n \in \mathbb{N}$,

$$
\begin{aligned}
& \mathbb{Z}_{n}^{d}=\mathbb{Z}^{d} / n \quad \text { (the rescaled lattice), } \\
& \left.\Omega_{n}=\left\{x \in \mathbb{Z}_{n}^{d} ; \mathrm{d}_{\infty}(x, \Omega)<1 / n\right\} \quad \text { (the discrete counterpart of } \Omega\right), \\
& \Gamma_{n}=\partial^{i n} \Omega_{n} \quad\left(\text { the inner vertex boundary of } \Omega_{n}\right) .
\end{aligned}
$$

The Ising-Potts model on $\boldsymbol{\Omega}$. Let $q \in \mathbb{N} \backslash\{0,1\}$. Consider a sequence $\gamma=$ $\Gamma^{1}, \ldots, \Gamma^{q}$ of $q$ disjoint and relatively open subsets of $\Gamma$ such that the relative boundary of $\Gamma \backslash \bigcup_{1 \leqslant i \leqslant q} \Gamma^{i}$ in $\Gamma$ has zero $\mathcal{H}^{d-1}$ measure. We set for $n \in \mathbb{N}$ and $i=1, \ldots, q$,

$$
\Gamma_{n}^{i}=\left\{x \in \Gamma_{n} ;\left(x, \Gamma^{i}\right)<1 / n \text { and } \forall j<i,\left(x, \Gamma^{j}\right) \geqslant 1 / n\right\} \quad i=1, \ldots, q .
$$

We use the sequence of $q$-tuples of sets $\gamma(n)=\left(\Gamma_{n}^{1}, \ldots, \Gamma_{n}^{q}\right)$ to specify boundary conditions as defined in Section 2.2; namely we impose $i$-b.c.s on $\Gamma_{n}^{i}$ for $i=1, \ldots, q$ and free b.c.s on $\Gamma_{n}^{0}=\Gamma_{n} \backslash \bigcup_{i=1, \ldots, q} \Gamma_{n}^{i}$. We denote the Ising-Potts measure in $\Omega_{n}$ with these b.c.s by $\mu_{n}=\mu_{n}^{\beta, q, \gamma(n)}$.

The typical picture which emerges from the Potts model at the macroscopic level is a partition of $\Omega$ in maximal $q$ phases corresponding to the dominant color in that phase. The individual phases need not to be connected. A convenient way to identify the phases is to look at the local density of the individual colors on an intermediate scale.

For $x \in \mathbb{R}^{d}$ and $r>0$ we define the box $\Lambda(x, r)$ by

$$
\Lambda(x, r)=\left\{y \in \mathbb{R}^{d} ;-r / 2<y_{i}-x_{i} \leqslant r / 2, i=1, \ldots, d\right\} .
$$

We next introduce an intermediate length scale represented by a fixed function $f: \mathbb{N} \rightarrow \mathbb{N}$ satisfying

$$
\lim _{n \rightarrow \infty} n / f(n)^{d-1}=\lim _{n \rightarrow \infty} f(n) / \log n=\infty .
$$

For $i=1, \ldots, q$, the local density of the color $i$ around $x \in \Omega$ is defined by

$$
\sigma_{n}(x, i)=f(n)^{-d}\left|\left\{y \in \Omega_{n} \cap \Lambda(x, f(n) / n) ; \sigma_{n}(y)=i\right\}\right|,
$$

where $\sigma_{n}(y)$ is the color of the vertex $y \in \Omega_{n}$ in the microscopic spin configuration $\sigma_{n}$ in $\Omega_{n}$. We partition $\Omega$ into the random sets $A_{n}^{i}, i=0,1, \ldots, q$, according to the value of the locally dominant color. More precisely, we set for $i=1, \ldots, q$,

$$
A_{n}^{i}=\left\{x \in \Omega ; \forall j \in\{1, \ldots, q\} \backslash\{i\}, \sigma_{n}(x, j)<\sigma_{n}(x, i)\right\}
$$

and $A_{n}^{0}$ is the set of those points where ties occur. The collection $\left(A_{n}^{0}, A_{n}^{1}, \ldots, A_{n}^{q}\right)$ is called the empirical phase partition. Our first result shows that up to super-surface order large deviations, the region $A_{n}^{0}$ has negligible density and the other regions are colored (magnetized) as in a pure phase corresponding to their dominant color, providing thereby evidence for sharp phase separation in Ising-Potts models. It is important to point out 
that the use of the spin-densities to identify the (pure) phases is not the only possibility. The proof shows that we could use any bulk quantity and verify that within the phases the correct values are taken, characteristic for the pure phases.

Theorem 1.1. - Let $d \geqslant 3, q \in \mathbb{N} \backslash\{0,1\}, \beta>\widehat{\beta}_{c}, \beta \in \mathcal{U}(q, d)$. For $\delta>0$,

$$
\begin{array}{r}
\limsup _{n \rightarrow \infty} \frac{1}{n^{d-1}} \log \mu_{n}\left\{\mathcal{L}^{d}\left(A_{n}^{0}\right)+\sum_{i=1, \ldots, q} \int_{A_{n}^{i}}\left(\left|\sigma_{n}(x, i)-\left(\theta+\frac{1-\theta}{q}\right)\right|\right.\right. \\
\left.\left.+\sum_{\substack{j=1, \ldots, q \\
j \neq i}}\left|\sigma_{n}(x, j)-\frac{1-\theta}{q}\right|\right) \mathrm{d} x>\delta\right\}=-\infty .
\end{array}
$$

Theorem 1.1 allows to relate the average densities of the $q$ different colors with the volumes of the sets $\left(A_{n}^{0}, \ldots, A_{n}^{q}\right)$. For $i$ in $\{1, \ldots, q\}$, let

$$
\mathcal{S}_{n}(i)=\left|\Omega_{n}\right|^{-1}\left|\left\{x \in \Omega_{n} ; \sigma_{n}(x)=i\right\}\right| .
$$

COROLlaRY 1.2. - For $i$ in $\{1, \ldots, q\}$, the sequences of random variables

$$
\left(\mathcal{S}_{n}(i)\right)_{n \in \mathbb{N}} \text { and }\left(\theta \mathcal{L}^{d}\left(A_{n}^{i}\right) / \mathcal{L}^{d}(\Omega)+(1-\theta) / q\right)_{n \in \mathbb{N}}
$$

are exponentially contiguous, i.e.,

$$
\forall \delta>0 \quad \limsup _{n \rightarrow \infty} \frac{1}{n^{d-1}} \log \mu_{n}\left[\left|\mathcal{S}_{n}(i)-\theta \mathcal{L}^{d}\left(A_{n}^{i}\right) / \mathcal{L}^{d}(\Omega)-(1-\theta) / q\right|>\delta\right]=-\infty .
$$

Proof. - Let us fix $i$ in $\{1, \ldots, q\}$. We write

$$
\begin{aligned}
\left|\mathcal{S}_{n}(i)-\theta \mathcal{L}^{d}\left(A_{n}^{i}\right) / \mathcal{L}^{d}(\Omega)-(1-\theta) / q\right| \\
\leqslant\left|\mathcal{S}_{n}(i)-\frac{n^{d}}{\left|\Omega_{n}\right|} \int_{\Omega} \sigma_{n}(x, i) \mathrm{d} x\right|+\left|\frac{n^{d} \mathcal{L}^{d}(\Omega)}{\left|\Omega_{n}\right|}-1\right| \\
\quad+\left|\frac{1}{\mathcal{L}^{d}(\Omega)} \int_{\Omega} \sigma_{n}(x, i) \mathrm{d} x-\theta \frac{\mathcal{L}^{d}\left(A_{n}^{i}\right)}{\mathcal{L}^{d}(\Omega)}-\frac{1-\theta}{q}\right| .
\end{aligned}
$$

We study successively each term of the right-hand side. Since $\Omega_{n}$ is a discretized version of $\Omega$, the second term goes to 0 as $n$ goes to $\infty$. Each point $x$ such that $\Lambda(x, f(n) / n) \subseteq \Omega$ belongs to $f(n)^{d}$ boxes of the form $\Lambda(y, f(n) / n), y \in \Omega$. Therefore

$$
\left|\mathcal{S}_{n}(i)-\frac{n^{d}}{\left|\Omega_{n}\right|} \int_{\Omega} \sigma_{n}(x, i) \mathrm{d} x\right| \leqslant \frac{2 n^{d}}{\left|\Omega_{n}\right|} \mathcal{L}^{d}\left(\left\{x \in \Omega: d_{2}(x, \Gamma)<2 d f(n) / n\right\}\right)+2 \frac{\left|\Gamma_{n}\right|}{\left|\Omega_{n}\right|} .
$$

Our hypothesis on $\Omega$ implies in particular that the boundary $\Gamma$ of $\Omega$ is $d-1$ rectifiable (in the terminology of Federer's book [26]) and closed, therefore its Minkowski content is equal to $\mathcal{H}^{d-1}(\Gamma)$, from which we deduce that the above term goes (deterministically) to 0 as $n$ goes to $\infty$. To deal with the third term, we write 


$$
\begin{aligned}
& \left|\int_{\Omega} \sigma_{n}(x, i) \mathrm{d} x-\theta \mathcal{L}^{d}\left(A_{n}^{i}\right)-\frac{1-\theta}{q} \mathcal{L}^{d}(\Omega)\right| \\
& \quad \leqslant \int_{A_{n}^{i}}\left|\sigma_{n}(x, i)-\theta-\frac{1-\theta}{q}\right| \mathrm{d} x+\sum_{j \neq i} \int_{A_{n}^{j}}\left|\sigma_{n}(x, j)-\frac{1-\theta}{q}\right| \mathrm{d} x+\mathcal{L}^{d}\left(A_{n}^{0}\right)
\end{aligned}
$$

and Theorem 1.1 provides the desired probabilistic control over this last term.

Our second result is a LDP for the empirical phase partition $\left(A_{n}^{0}, A_{n}^{1}, \ldots, A_{n}^{q}\right)$. We first define a (pseudo) metric dist $L^{1}$ on the set $\mathcal{B}(\Omega)$ of the Borel subsets of $\Omega$ by setting

$$
\forall A_{1}, A_{2} \in \mathcal{B}(\Omega) \quad \operatorname{dist}_{L^{1}}\left(A_{1}, A_{2}\right)=\mathcal{L}^{d}\left(A_{1} \Delta A_{2}\right) .
$$

We consider then the space of phase partitions $P(\Omega, q)$ consisting of $(q+1)$-tuples $\left(A^{0}, A^{1}, \ldots, A^{q}\right)$ of Borel subsets of $\Omega$ forming a partition of $\Omega$. We endow $P(\Omega, q)$ with the following metric:

$$
\operatorname{dist}_{P}\left(\left(A^{0}, \ldots, A^{q}\right),\left(B^{0}, \ldots, B^{q}\right)\right)=\sum_{i=0, \ldots, q} \operatorname{dist}_{L^{1}}\left(A^{i}, B^{i}\right) .
$$

The surface energy $\mathcal{I}$ of a phase partition $\left(A_{n}^{0}, A_{n}^{1}, \ldots, A_{n}^{q}\right) \in P(\Omega, q)$ is defined as follows:

- for any $\left(A^{0}, A^{1}, \ldots, A^{q}\right)$ such that either $A^{0} \neq \emptyset$ or one set among $A^{1}, \ldots, A^{q}$ has not finite perimeter, we set $\mathcal{I}\left(A^{0}, \ldots, A^{q}\right)=\infty$

- for any $\left(A^{0}, A^{1}, \ldots, A^{q}\right)$ with $A^{0}=\emptyset$ and $A^{1}, \ldots, A^{q}$ having finite perimeter we set

$$
\begin{aligned}
\mathcal{I}\left(A^{0}, \ldots, A^{q}\right)= & \sum_{i=1, \ldots, q} \frac{1}{2} \int_{\partial^{*} A^{i} \cap \Omega} \tau\left(v_{A_{i}}(x)\right) \mathrm{d} \mathcal{H}^{d-1}(x) \\
& +\sum_{\substack{i, j=1, \ldots, q \\
i \neq j}} \int_{\partial^{*} A^{i} \cap \Gamma^{j}} \tau\left(v_{A_{i}}(x)\right) \mathrm{d} \mathcal{H}^{d-1}(x) .
\end{aligned}
$$

Note that $\mathcal{I}$ depends on $\tau$ and the boundary conditions $\gamma=\left(\Gamma^{1}, \ldots, \Gamma^{q}\right)$. The first term in the above formula corresponds to the interfaces present in $\Omega$, while the second term corresponds to the interfaces between the elements of the phase partition and the boundary $\Gamma$. For a set $A$ of finite perimeter, $\partial^{*} A$ denotes its reduced boundary (see the Appendix).

Results related to the lower semicontinuity of functionals more general than $\mathcal{I}$ and the theory of their $\Gamma$-convergence can be found in $[5,6]$. In particular, $\mathcal{I}$ is a good rate function on the space $\left(P(\Omega, q)\right.$, dist $\left.{ }_{P}\right)$, i.e., it is lower semicontinuous and it has compact level sets.

Let $\min _{P(\Omega, q)} \mathcal{I}$ be the minimum value of $\mathcal{I}$ over $P(\Omega, q)$. Clearly this minimum is always finite.

THEOREM 1.3. - The sequence $\left(\vec{A}_{n}\right)_{n \in \mathbb{N}}=\left(\left(A_{n}^{0}, A_{n}^{1}, \ldots, A_{n}^{q}\right)\right)_{n \in \mathbb{N}}$ of the empirical phase partitions of $\Omega$ satisfies a LDP in $\left(P(\Omega, q)\right.$, dist $\left.P_{P}\right)$ with respect to $\mu_{n}$ with speed $n^{d-1}$ and rate function $\mathcal{I}-\min _{P(\Omega, q)} \mathcal{I}$, i.e., for any Borel subset $\mathbb{E}$ of $P(\Omega, q)$, 


$$
\begin{aligned}
-\inf _{\mathbb{E}} \mathcal{I}+\min _{P(\Omega, q)} \mathcal{I} & \leqslant \liminf _{n \rightarrow \infty} \frac{1}{n^{d-1}} \log \mu_{n}\left[\vec{A}_{n} \in \mathbb{E}\right] \leqslant \limsup _{n \rightarrow \infty} \frac{1}{n^{d-1}} \log \mu_{n}\left[\vec{A}_{n} \in \mathbb{E}\right] \\
& \leqslant-\inf _{\mathbb{E}} \mathcal{I}+\min _{P(\Omega, q)} \mathcal{I} .
\end{aligned}
$$

Remark. - The constant $\min _{P(\Omega, q)} \mathcal{I}$ will be related to another quantity defined at the FK level in Lemma 1.7.

Recall that imposing mixed boundary conditions is not the only way to force the system to exhibit coexisting phases. In the Wulff problem in the Ising model context, for instance, a restricted ensemble is studied which is characterized by an artificial excess of say minus spins in the plus phase. Technically this can be achieved by conditioning the system to have a magnetization larger than the spontaneous magnetization while imposing plus b.c.s.

The next result describes the large deviation behavior of the phase partition in a large class of restricted ensembles. Although it is a rather straightforward generalization of Theorem 1.3, we state it separately because of its physical relevance.

Let $\left(G_{n}\right)_{n \geqslant 1}$ be a sequence of events, i.e., sets of spin configurations, satisfying the following two conditions: first there exists a Borel subset $\mathbb{G}$ of $P(\Omega, q)$ such that the sequence of events $\left(G_{n}\right)_{n \in \mathbb{N}}$ and $\left(\left\{\vec{A}_{n} \in \mathbb{G}\right\}\right)_{n \in \mathbb{N}}$ are exponentially equivalent, i.e.,

$$
\limsup _{n \rightarrow \infty} \frac{1}{n^{d-1}} \log \mu_{n}\left[G_{n} \triangle\left\{\vec{A}_{n} \in \mathbb{G}\right\}\right]=-\infty,
$$

where $\Delta$ denotes the symmetric difference. Second, the following limit exists and is finite:

$$
\mathcal{I}_{G}=\lim _{n \rightarrow \infty} \frac{1}{n^{d-1}} \log \mu_{n}\left[G_{n}\right]>-\infty .
$$

The sequence of events $\left(G_{n}\right)_{n \geqslant 1}$ determines a restricted (conditional) ensemble. Note that if

$$
\inf _{\mathbb{G}} \mathcal{I}=\inf _{\overline{\mathbb{G}}} \mathcal{I}>-\infty
$$

then Theorem 1.3 implies that (4) is satisfied, with $\mathcal{I}_{G}=\inf _{\mathbb{G}} \mathcal{I}$.

THEOREM 1.4. - Assume that the sequence $\left(G_{n}\right)_{n \geqslant 1}$ satisfies (3) and (4) and define for each $n \geqslant 1$ the conditional measures

$$
\mu_{n}^{G}=\mu_{n}\left(\cdot \mid G_{n}\right) .
$$

Then the sequence $\left(\vec{A}_{n}\right)_{n \geqslant 1}$ of the empirical phase partitions of $\Omega$ satisfies a LDP in $\left(P(\Omega, q), \operatorname{dist}_{P}\right)$ with respect to $\mu_{n}^{G}$ with speed $n^{d-1}$ and rate function $\mathcal{I}-\mathcal{I}_{G}$, i.e., for any Borel subset $\mathbb{E}$ of $P(\Omega, q)$,

$$
\begin{aligned}
-\inf _{\mathbb{E} \cap \mathbb{G}} \mathcal{I}+\mathcal{I}_{G} & \leqslant \liminf _{n \rightarrow \infty} \frac{1}{n^{d-1}} \log \mu_{n}^{G}\left[\vec{A}_{n} \in \mathbb{E}\right] \leqslant \limsup _{n \rightarrow \infty} \frac{1}{n^{d-1}} \log \mu_{n}^{G}\left[\vec{A}_{n} \in \mathbb{E}\right] \\
& \leqslant-\inf _{\mathbb{E} \cap \mathbb{G}} \mathcal{I}+\mathcal{I}_{G} .
\end{aligned}
$$


Theorem 1.4 gives a rigorous verification of the basic assumption underlying the phenomenological theory, namely, that in a given ensemble, the typical configurations are those minimizing the surface free energy.

We show next how Theorem 1.4 can be applied to the Wulff and multiple bubble problem. We take pure boundary conditions with color 1 , that is, $\Gamma^{1}=\Gamma, \Gamma^{2}=\cdots=$ $\Gamma^{q}=\emptyset$. Let $s_{2}, \ldots, s_{q}$ be $q-1$ real numbers larger than or equal to $(1-\theta) / q$. We set

$$
\forall i \in\{2, \ldots, q\} \quad v_{i}=\mathcal{L}^{d}(\Omega) \theta^{-1}\left(s_{i}-(1-\theta) / q\right) .
$$

We define next the events

$$
\forall n \in \mathbb{N} \quad G_{n}=\left\{\forall i \in\{2, \ldots, q\} \mathcal{S}_{n}(i) \geqslant s_{i}\right\}
$$

and the collection of phase partitions

$$
\mathbb{G}\left(v_{2}, \ldots, v_{q}\right)=\left\{\vec{A}=\left(A_{0}, A_{1}, \ldots, A_{q}\right) \in P(\Omega, q): \mathcal{L}^{d}\left(A_{2}\right) \geqslant v_{2}, \ldots, \mathcal{L}^{d}\left(A_{q}\right) \geqslant v_{q}\right\} .
$$

Corollary 1.2 implies that the sequences of events

$$
\left(G_{n}\right)_{n \in \mathbb{N}} \text { and }\left(\vec{A}_{n} \in \mathbb{G}\left(v_{2}, \ldots, v_{q}\right)\right)_{n \in \mathbb{N}}
$$

are exponentially contiguous, i.e., they satisfy the condition (3). In order to ensure condition (5), we suppose that the minimum of the surface energy $\mathcal{I}$ over $\mathbb{G}\left(v_{2}, \ldots, v_{q}\right)$ is reached with a phase partition having no interfaces on the boundary $\Gamma$. More precisely, we suppose that the following assumption is fulfilled.

Assumption. - The region $\Omega$ and the real numbers $v_{2}, \ldots, v_{q}$ are such that there exists $\vec{A}^{*}=\left(A_{0}^{*}, A_{1}^{*}, \ldots, A_{q}^{*}\right)$ in $\mathbb{G}\left(v_{2}, \ldots, v_{q}\right)$ such that

$$
\begin{gathered}
\mathcal{I}\left(\vec{A}^{*}\right)=\min \left\{\mathcal{I}(\vec{A}) ; \vec{A} \in \mathbb{G}\left(v_{2}, \ldots, v_{q}\right)\right\}, \\
\forall i \in\{2, \ldots, q\}, \quad d_{2}\left(A_{i}^{*}, \Gamma\right)>0 .
\end{gathered}
$$

We expect that this assumption is fulfilled provided the real numbers $v_{2}, \ldots, v_{q}$ are sufficiently small (or equivalently, $s_{2}, \ldots, s_{q}$ are sufficiently close to $(1-\theta) / q$ ), depending on the region $\Omega$. This is for instance the case when $q=2$. Indeed, let $\mathcal{W}_{\tau}$ be the Wulff crystal associated to $\tau$. We know that $\mathcal{W}_{\tau}$ is, up to dilatations and translations, the unique solution to the anisotropic isoperimetric problem associated to $\tau$. For $v_{2}$ sufficiently small, a dilated Wulff crystal $x_{0}+\alpha_{0} \mathcal{W}_{\tau}$ of volume $v_{2}$ fits into $\Omega$ without touching $\Gamma$, and the phase partition $\vec{A}^{*}=\left(\emptyset, \Omega \backslash\left(x_{0}+\alpha_{0} \mathcal{W}_{\tau}\right), x_{0}+\alpha_{0} \mathcal{W}_{\tau}\right)$ answers the problem. In the case $q>2$, we expect that a minimizing phase partition corresponds to a multiple bubble having $q-1$ components.

Under the above assumption, we claim that the collection of phase partitions $\mathbb{G}\left(v_{2}, \ldots, v_{q}\right)$ satisfies (5). For $\lambda>1$, we define

$$
\vec{A}^{*}(\lambda)=\left(\emptyset, \Omega \backslash \bigcup_{2 \leqslant i \leqslant q} \lambda A_{i}^{*}, \lambda A_{2}^{*}, \ldots, \lambda A_{q}^{*}\right) .
$$


Since by hypothesis the sets $A_{2}^{*}, \ldots, A_{q}^{*}$ are at positive distance from $\Gamma$, for $\lambda$ larger than 1 and sufficiently close to 1 , the phase partition $\vec{A}^{*}(\lambda)$ satisfies

$$
\vec{A}^{*}(\lambda) \in \mathbb{G}\left(\lambda^{d} v_{2}, \ldots, \lambda^{d} v_{q}\right) \subset \mathbb{G}\left(v_{2}, \ldots, v_{q}\right)
$$

and moreover $\mathcal{I}\left(\vec{A}^{*}(\lambda)\right)=\lambda^{d-1} \mathcal{I}\left(\vec{A}^{*}\right)$. Sending $\lambda$ to 1 , and remarking that $\mathbb{G}\left(v_{2}, \ldots, v_{q}\right)$ is closed, we see that $\mathbb{G}\left(v_{2}, \ldots, v_{q}\right)$ satisfies (5). Thus we can apply Theorem 1.4 with the sequence of events $\left(G_{n}\right)_{n \in \mathbb{N}}$, thereby obtaining a LDP and a weak law of large numbers for the conditional measures $\mu_{n}^{G}=\mu_{n}\left(\cdot \mid G_{n}\right)$. In the particular case $q=2$, we obtain again the main result of our previous paper [15]. In the more challenging situations $q>2$, the unresolved questions concerning the macroscopic behavior of such systems belong to the realm of the calculus of variations.

Ising model. For the reader's convenience, we rephrase our basic results in the Ising setting. In this case $q=2$ and the phases 1 and 2 are usually called + and - phases. We use the same notation as in the Potts case except that 1 will be replaced by + and 2 by - . For instance we write $\Gamma_{n}^{+}$instead of $\Gamma_{n}^{1}$ and $\Omega_{n}^{-}$instead of $A_{n}^{2}$. The index 0 remains. In this case the spontaneous magnetization can be given as $m^{*}(\beta)=\theta^{w}(p, 2)$ with $p=1-\mathrm{e}^{-\beta}$. The locally averaged magnetization $\sigma_{n}$ is the map from $\Omega$ to $[-1,1]$ defined by

$$
\forall x \in \Omega \quad \sigma_{n}(x)=\frac{1}{f(n)^{d}} \sum_{y \in \Lambda(x, f(n) / n) \cap \Omega_{n}} \sigma(y) .
$$

We partition $\Omega$ into the random sets $\Omega_{n}^{-}, \Omega_{n}^{0}$ and $\Omega_{n}^{+}$according to whether the value of the local magnetization is smaller, equal or larger than zero.

COROLlary 1.5. - Let $d \geqslant 3, \beta>\widehat{\beta}_{c}, \beta \in \mathcal{U}(2, d)$. For $\delta>0$,

$$
\limsup _{n \rightarrow \infty} \frac{1}{n^{d-1}} \log \mu_{n}\left[\int_{\Omega_{n}^{-}}\left|\sigma_{n}(x)+m^{*}\right| \mathrm{d} x+\mathcal{L}^{d}\left(\Omega_{n}^{0}\right)+\int_{\Omega_{n}^{+}}\left|\sigma_{n}(x)-m^{*}\right| \mathrm{d} x>\delta\right]=-\infty \text {. }
$$

The surface energy $\mathcal{I}$ on $P(\Omega, 2)$ is given as follows. If $A^{0} \neq \emptyset$ or if $\mathcal{P}\left(A^{-}\right)+$ $\mathcal{P}\left(A^{+}\right)=\infty$, we set $\mathcal{I}\left(A^{0}, A^{-}, A^{+}\right)=\infty$. For any $\left(A^{0}, A^{-}, A^{+}\right)$with $A^{0}$ and $A^{-}, A^{+}$ having finite perimeter we set

$$
\begin{aligned}
\mathcal{I}\left(A^{0}, A^{-}, A^{+}\right)= & \frac{1}{2} \int_{\partial^{*} A^{-} \cap \Omega} \tau\left(v_{A_{-}}(x)\right) \mathrm{d} \mathcal{H}^{d-1}(x)+\int_{\partial^{*} A^{-} \cap \Gamma^{+}} \tau\left(v_{A_{-}}(x)\right) \mathrm{d} \mathcal{H}^{d-1}(x) \\
& +\frac{1}{2} \int_{\partial^{*} A^{+} \cap \Omega} \tau\left(v_{A_{+}}(x)\right) \mathrm{d} \mathcal{H}^{d-1}(x)+\int_{\partial^{*} A^{+} \cap \Gamma^{-}} \tau\left(v_{A_{+}}(x)\right) \mathrm{d} \mathcal{H}^{d-1}(x) .
\end{aligned}
$$

COROLLARY 1.6. - The sequence $\left(\vec{\Omega}_{n}\right)_{n \in \mathbb{N}}=\left(\left(\Omega_{n}^{0}, \Omega_{n}^{-}, \Omega_{n}^{+}\right)\right)_{n \in \mathbb{N}}$ of the empirical phase partitions of $\Omega$ satisfies a LDP in $\left(P(\Omega, 2)\right.$, dist $\left.P_{P}\right)$ with respect to $\mu_{n}$ with speed $n^{d-1}$ and rate function $\mathcal{I}-\min _{P(\Omega, 2)} \mathcal{I}$, i.e., for any Borel subset $\mathbb{E}$ of $P(\Omega, 2)$,

$$
\begin{aligned}
-\inf _{\mathbb{E}} \mathcal{I}+\min _{P(\Omega, 2)} \mathcal{I} & \leqslant \liminf _{n \rightarrow \infty} \frac{1}{n^{d-1}} \log \mu_{n}\left[\vec{\Omega}_{n} \in \mathbb{E}\right] \leqslant \limsup _{n \rightarrow \infty} \frac{1}{n^{d-1}} \log \mu_{n}\left[\vec{\Omega}_{n} \in \mathbb{E}\right] \\
& \leqslant-\inf _{\overline{\mathbb{E}}} \mathcal{I}+\min _{P(\Omega, 2)} \mathcal{I} .
\end{aligned}
$$


FK model. Consider a sequence $\gamma=\left(\Gamma^{m}\right)_{m \in \mathbb{N}}$ of (possibly empty) disjoint and relatively open subsets of $\Gamma$. The relative boundary of $\Gamma \backslash \bigcup_{m} \Gamma^{m}$ in $\Gamma$ should have zero $\mathcal{H}^{d-1}$ measure. We set for $n \in \mathbb{N}$

$$
\Gamma_{n}^{m}=\left\{x \in \Gamma_{n} ; \mathrm{d}_{\infty}\left(x, \Gamma^{m}\right)<1 / n, \forall \ell<m, \mathrm{~d}_{\infty}\left(x, \Gamma^{\ell}\right) \geqslant 1 / n\right\}, \quad m \in \mathbb{N} .
$$

We use the sequence $\left(\Gamma_{n}^{m}\right)_{m \in \mathbb{N}}$ to specify boundary conditions for FK percolation in the following way: for each $m \in \mathbb{N}$, the points belonging to $\Gamma_{n}^{m}$ are wired together, while the points in $\Gamma_{n} \backslash \bigcup_{m} \Gamma_{n}^{m}$ are let free. FK clusters are regarded to be connected (hence identical) when they contain sites which are wired together, i.e., if they intersect the same boundary piece $\Gamma_{n}^{m}$ for some $m$.

Let $\gamma(n)$ be the partition of $\Gamma_{n}$ consisting of the sequence $\left(\Gamma_{n}^{m}\right)_{m \in \mathbb{N}}$ together with the singletons $\{x\}, x \in \Gamma_{n} \backslash \bigcup_{m} \Gamma_{n}^{m}$. The FK measure inside $\Omega$ with partially wired boundary conditions induced by the sequence $\left(\Gamma^{m}\right)_{m \in \mathbb{N}}$ with lattice spacing $1 / n$ is the measure $\Phi_{n}$ given by

$$
\Phi_{n}=\Phi_{\Omega_{n}}^{p, q, \gamma(n)} .
$$

Our principal result, Theorem 1.8, describes a LDP for the collection of the large FK clusters in the FK model which correspond to several coexisting pure phases in the spin language. To deal with the entire collection of FK clusters simultaneously we use Borel partitions (to be defined below) in a similar way as they were employed in [14]. In the spin setting the framework of partitions allows us to describe systems with more than two phases on the macroscopic level. Regarding the applications to Ising-Potts models with mixed boundary conditions we have to refine the structure of partitions in order to keep track of the microscopic connections (whose absence indicates an interface) between large clusters and the wired boundary pieces. We achieve this by introducing a touching function $\mathcal{T}(A, m)$ indicating the presence or absence of a "connection" between $\Gamma^{m}$ and a given set $A \subseteq \Omega$. Given a partition and its "touching status" there is a natural way to assign a surface energy to it with respect to the surface tension $\tau$. In the next paragraphs we give a brief description of these constructions so that we are able to formulate Theorem 1.8.

A Borel partition $\mathcal{A}$ of $\Omega$ is a finite or countable collection of non-negligible Borel subsets of $\Omega$ which, up to negligible sets, form a partition of $\Omega$. The perimeter $\mathcal{P}(\mathcal{A})$ of a partition $\mathcal{A}$ is defined as

$$
\mathcal{P}(\mathcal{A})=\sum_{A \in \mathcal{A}} \mathcal{P}(A)
$$

where $\mathcal{P}(A)$ is the classical perimeter of $A$ (see the appendix).

A touching function associated with a partition $\mathcal{A}$ of $\Omega$ is a map $\mathcal{T}: \mathcal{A} \times \mathbb{N} \mapsto\{0,1\}$ describing contacts between the sets of $\mathcal{A}$ and the boundary pieces $\left(\Gamma^{m}\right)_{m \in \mathbb{N}}$; for $m \in \mathbb{N}$, a set $A$ of $\mathcal{A}$ is said to touch a boundary piece $\Gamma^{m}$ if and only if $\mathcal{T}(A, m)=1$. Thus there is no microscopic connection between $A$ and $\Gamma^{m}$ if $\mathcal{T}(A, m)=0$.

To define FK clusters we identify the physical clusters intersecting the same piece $\Gamma_{n}^{m}$ of the discretized boundary. As a consequence, a touching function must satisfy the following compatibility condition: a boundary piece $\Gamma^{m}$ can touch at most one set of the partition. 
A $t$-partition is a pair $(\mathcal{A}, \mathcal{T})$ where $\mathcal{A}$ is a partition of $\Omega$ and $\mathcal{T}$ is a touching function associated with $\mathcal{A}$. We denote by $\operatorname{TP}(\Omega)$ the set of all $t$-partitions of $\Omega$ with finite perimeter.

Our next goal is to define an appropriate metric on the space $\operatorname{TP}(\Omega)$. We first define a metric dist $\mathcal{F}$ to deal with touching functions. Let $\mathcal{F}$ be the set of the functions from $\mathbb{N}$ to $\{0,1\}$ endowed with the product topology. This topology is metrizable, for instance it is compatible with the metric dist $\mathcal{F}$ defined by

$$
\forall T_{1}, T_{2} \in \mathcal{F} \quad \operatorname{dist}_{\mathcal{F}}\left(T_{1}, T_{2}\right)=\sum_{m \in \mathbb{N}} 2^{-m}\left|T_{1}(m)-T_{2}(m)\right| .
$$

An arrangement of an element $(\mathcal{A}, \mathcal{T})$ of $\operatorname{TP}(\Omega)$ is a sequence $(A(i), T(i, \cdot), i \in \mathbb{N})$ of sets in $\mathcal{A} \cup\{\emptyset\}$ and functions in $\mathcal{F}$ such that:

- each set of $\mathcal{A}$ appears exactly once in the sequence $(A(i), i \in \mathbb{N})$ and the empty set $\emptyset$ appears countably many times in the sequence $(A(i), i \in \mathbb{N})$.

- for any $i \in \mathbb{N}$, if $A(i) \neq \emptyset$, then $T(i, \cdot)=\mathcal{T}(A(i), \cdot)$.

In particular, if $\mathcal{A}$ is finite, then $A(i)=\emptyset$ for $i$ sufficiently large. Whenever $A(i)=\emptyset$, the corresponding function $T(i, \cdot)$ might be any element of $\mathcal{F}$. However we impose the global constraint that a boundary piece can touch at most one set, that is,

$$
\forall m \in \mathbb{N} \quad \sum_{i \in \mathbb{N}} T(i, m) \leqslant 1 .
$$

Finally we define a metric Dist on $\operatorname{TP}(\Omega)$ as follows: for $\left(\mathcal{A}_{1}, \mathcal{T}_{1}\right),\left(\mathcal{A}_{2}, \mathcal{T}_{2}\right) \in \operatorname{TP}(\Omega)$ $\operatorname{Dist}\left(\left(\mathcal{A}_{1}, \mathcal{T}_{1}\right),\left(\mathcal{A}_{2}, \mathcal{T}_{2}\right)\right)=\inf \left\{\sum_{i \in \mathbb{N}}\left(\operatorname{dist}_{L^{1}}\left(A_{1}(i), A_{2}(i)\right)+\operatorname{dist}_{\mathcal{F}}\left(T_{1}(i, \cdot), T_{2}(i, \cdot)\right)\right)\right\}$, where the infimum is taken over all possible arrangements $\left(A_{j}(i), T_{j}(i, \cdot), i \in \mathbb{N}\right)$ of $\mathcal{A}_{j}$, $j=1,2$, and dist $L^{1}$ was defined in (2).

Remark. - If we forget about the touching function, the metric Dist is the one used by Congedo and Tamanini [16-18] (which is stronger than the one employed in [14]). For a careful exposition and study of this metric on the space of Caccioppoli partitions, see [40].

The surface energy (depending on $\tau$ and on the boundary conditions $\gamma$ ) of a $t$-partition in $\operatorname{TP}(\Omega)$ is defined as

$$
\begin{aligned}
\mathcal{I}(\mathcal{A}, \mathcal{T})= & \sum_{A \in \mathcal{A}}\left(\frac{1}{2} \int_{\partial^{*} A \cap \Omega} \tau\left(v_{A}(x)\right) \mathrm{d} \mathcal{H}^{d-1}(x)\right. \\
& \left.+\sum_{m \in \mathbb{N}}(1-\mathcal{T}(A, m)) \int_{\partial^{*} A \cap \Gamma^{m}} \tau\left(v_{A}(x)\right) \mathrm{d} \mathcal{H}^{d-1}(x)\right) .
\end{aligned}
$$

The first term in the above formula corresponds to the interfaces present within $\Omega$, while the second term corresponds to the interfaces between the elements of the partition and the boundary $\Gamma$. It is instructive to express now the constant appearing in the LDP of Theorem 1.3 with the help of the above surface energy. 
LEMMA 1.7. - For the sequence $\left(\Gamma^{m}\right)_{m \in \mathbb{N}}$, we choose here the finite sequence $\Gamma^{1}, \ldots, \Gamma^{q}$ used in the section on the Potts model. Let $\mathcal{F}$ denote the set of t-partitions such that no set touches simultaneously two distinct boundary parts, i.e.,

$$
\mathcal{F}=\left\{(\mathcal{A}, \mathcal{T}) \in \operatorname{TP}(\Omega) ; \forall A \in \mathcal{A} \sum_{\substack{1 \leqslant i, j \leqslant q \\ i \neq j}} \mathcal{T}(A, i) \mathcal{T}(A, j)=0\right\} .
$$

Then $\min \{\mathcal{I}(\mathcal{A}, \mathcal{T}) ;(\mathcal{A}, \mathcal{T}) \in \mathcal{F}\}=\min \{\mathcal{I}(\vec{A}) ; \vec{A} \in P(\Omega, q)\}$.

Proof. - The argument is a straightforward consequence of the definitions of the two rate functions on the spaces $\operatorname{TP}(\Omega)$ and $P(\Omega, q)$ respectively. Indeed, let $(\mathcal{A}, \mathcal{T})$ belong to $\mathcal{F}$. For $i=1, \ldots, q$, there exists at most one element $A_{i}$ of $\mathcal{A}$ such that $\mathcal{T}\left(A_{i}, i\right)=1$. If there is no such element in $\mathcal{A}$, we set $A_{i}=\emptyset$. Let $\mathcal{A}_{0}=\mathcal{A} \backslash\left\{A_{1}, \ldots, A_{q}\right\}$. Let $\vec{A}$ be the phase partition defined by

$$
\vec{A}=\left(\emptyset, A_{1}, \ldots, A_{q-1}, A_{q} \cup \bigcup_{A \in \mathcal{A}_{0}} A\right) .
$$

Then $\mathcal{I}(\vec{A}) \leqslant \mathcal{I}(\mathcal{A}, \mathcal{T})$. Therefore $\min _{\mathcal{F}} \mathcal{I} \geqslant \min _{P(\Omega, q)} \mathcal{I}$. Conversely, let $\vec{A}=(\emptyset$, $\left.A_{1}, \ldots, A_{q}\right) \in P(\Omega, q)$. Let $(\mathcal{A}, \mathcal{T})$ be the element of $\operatorname{TP}(\Omega)$ defined by $\mathcal{A}=$ $\left\{A_{1}, \ldots, A_{q}\right\}$ and

$$
\forall i, j=1, \ldots, q \quad \mathcal{T}\left(A_{i}, j\right)= \begin{cases}1 ; & \text { if } i=j \\ 0 ; & \text { otherwise }\end{cases}
$$

$\mathcal{I}(\mathcal{A}, \mathcal{T})=\mathcal{I}(\vec{A})$ whence $\min _{\mathcal{F}} \mathcal{I} \leqslant \min _{P(\Omega, q)} \mathcal{I}$.

Now we are ready to turn to our basic LDP for FK percolation. Let $f: \mathbb{N} \rightarrow \mathbb{N}$ be our fixed function representing an intermediate length scale satisfying (1). For given $n$, a (physical) cluster $C$ on $\Omega_{n}$ is called large if $\operatorname{diam} C>f(n)$ and small otherwise. Let $\mathcal{C}_{n}$ denote the random collection of the large clusters. With $\mathcal{C}_{n}$ we associate the Voronoi partition of $\Omega$ with parts

$$
\text { vor } C=\left\{x \in \Omega ; \forall C^{\prime} \in \mathcal{C}_{n} \backslash\{C\}, \mathrm{d}_{2}\left(x, C^{\prime}\right)>\mathrm{d}_{2}(x, C)\right\}, \quad C \in \mathcal{C}_{n} .
$$

Recall that an FK cluster can be regarded as the union of clusters intersecting sites on the boundary which are wired. We define a large FK cluster as an FK cluster which contains at least one large cluster. The collection of all large FK clusters is denoted by $\mathcal{C}_{n}^{\mathrm{FK}}$. A generic element of $\mathcal{C}_{n}^{\mathrm{FK}}$ will be denoted by $C^{\mathrm{FK}}$. The Voronoi partition of $\Omega$ induced by $\mathcal{C}_{n}^{\mathrm{FK}}$ consists of the sets

$$
\operatorname{vor} C^{\mathrm{FK}}=\bigcup_{C \in \mathcal{C}_{n}, C \subseteq C^{\mathrm{FK}}} \operatorname{vor} C, \quad C^{\mathrm{FK}} \in \mathcal{C}_{n}^{\mathrm{FK}} .
$$

Finally, we associate with $\mathcal{C}_{n}^{\mathrm{FK}}$ the empirical t-partition $\left(\mathcal{A}_{n}, \mathcal{T}_{n}\right) \in \operatorname{TP}(\Omega)$ as follows: $\mathcal{A}_{n}$ is the Voronoi partition of $\Omega$ induced by $\mathcal{C}_{n}^{\mathrm{FK}}$ and $\mathcal{T}_{n}$ is the touching function 
determined by the existence or absence of connections between the large FK clusters and the boundary pieces. More precisely,

$$
\mathcal{A}_{n}=\left\{\operatorname{vor} C^{\mathrm{FK}} ; C^{\mathrm{FK}} \in \mathcal{C}_{n}^{\mathrm{FK}}\right\}
$$

and for any $m \in \mathbb{N}$, any $C^{\mathrm{FK}} \in \mathcal{C}_{n}^{\mathrm{FK}}$,

$$
\mathcal{T}_{n}\left(\operatorname{vor} C^{\mathrm{FK}}, m\right)= \begin{cases}1 ; & \text { if } C^{\mathrm{FK}} \cap \Gamma_{n}^{m} \neq \emptyset \\ 0 ; & \text { otherwise. }\end{cases}
$$

THEOREM 1.8. - Let $d \geqslant 3, q \geqslant 1, p>\widehat{p}_{c}$ such that $\theta^{f}(p)=\theta^{w}(p)$. The law of the empirical t-partition $\left(\mathcal{A}_{n}, \mathcal{T}_{n}\right)$ under the $F K$ measure $\Phi_{n}$ satisfies a large deviation principle in the metric space $\left(\operatorname{TP}(\Omega)\right.$, Dist) with speed $n^{d-1}$ and rate function the surface energy $\mathcal{I}$, i.e., for any Borel subset $\mathcal{E}$ of $(\mathrm{TP}(\Omega)$, Dist $)$,

$$
\begin{aligned}
-\inf _{\mathcal{E}} \mathcal{I} & \leqslant \liminf _{n \rightarrow \infty} \frac{1}{n^{d-1}} \log \Phi_{n}\left[\left(\mathcal{A}_{n}, \mathcal{T}_{n}\right) \in \mathcal{E}\right] \leqslant \limsup _{n \rightarrow \infty} \frac{1}{n^{d-1}} \log \Phi_{n}\left[\left(\mathcal{A}_{n}, \mathcal{T}_{n}\right) \in \mathcal{E}\right] \\
& \leqslant-\inf _{\overline{\mathcal{E}}} \mathcal{I} .
\end{aligned}
$$

Remark. - The LDP of Theorem 1.8 holds with a slightly weaker hypothesis on $f(n)$, namely: there exists a constant $\kappa=\kappa(d, p, q)$ such that for any function $f(n)$ satisfying

$$
\forall n \in \mathbb{N} \quad f(n) \geqslant \kappa \log n, \quad \lim _{n \rightarrow \infty} n / f(n)^{d-1}=\infty .
$$

the LDP stated in Theorem 1.8 holds. However, to transfer the LDP from the FK level to the spin level we work with the stronger hypothesis on $f(n)$.

The LDP stated in Theorem 1.8 is our most general LDP. In fact, we deduce the other LDPs from it. In the most general situation, this LDP ensures the concentration of the law of the system (under arbitrary conditions) near the minima of the associated variational problem. Since the rate function is good, the set of minima is never empty. However, in general, we have very little information on the minima themselves. A noticeable exception is for instance the Wulff problem, which we handled in [15] for the Ising model.

We finish with a straightforward consequence of the LDP of Theorem 1.8.

Corollary 1.9. - Let $d \geqslant 3, q \in \mathbb{N} \backslash\{0,1\}, \beta>\widehat{\beta}_{c}, \beta \in \mathcal{U}(q, d)$. Then our definition of surface tension in the FK model coincides with the classical definition of surface tension in the spin setting.

Proof. - Let $v$ be a unit vector in $\mathbb{R}^{d}$ and let $i<j$ be two different colors. We apply the LDP of Theorem 1.8 to the following situation: $\Omega=\Lambda(0,1)$ is a unit box centered at the origin, $\Gamma=\partial \Lambda(0,1)$ and for the b.c.s we consider the two sets $\Gamma^{+}=\{x \in \Gamma: x \cdot v>0\}$, $\Gamma^{-}=\{x \in \Gamma: x \cdot v<0\}$. Let $\tau^{\text {classic }}$ be the classical definition of surface tension in the spin setting for the Potts model (which is the limit of the excess free energy when putting for b.c.s. the color $i$ on $\Gamma^{-}$and the color $j$ on $\left.\Gamma^{+}\right)$. It is known that $\tau^{\text {classic }}(v)$ can be 
rewritten as (see for instance [12])

$$
\tau^{\text {classic }}(v)=-\lim _{n \rightarrow \infty} \frac{1}{n^{d-1} \mathcal{H}^{d-1}(S)} \log \Phi_{n}\left[\text { there is no open path between } \Gamma_{n}^{-} \text {and } \Gamma_{n}^{+}\right],
$$

where $S=\{x \in \Lambda(0,1) ; x \cdot v=0\}$. By Lemmas 1.7 and 4.14 , the above limit is equal to

$$
\mathcal{H}^{d-1}(S) \tau^{\text {classic }}(v)=\min \{\mathcal{I}(\vec{A}) ; \vec{A} \in P(\Omega, q)\} .
$$

Let $E_{-}=\left\{x \in \mathbb{R}^{d}: x \cdot v<0\right\}$ and $E_{+}=\left\{x \in \mathbb{R}^{d}: x \cdot v>0\right\}$. Let $\vec{A}=\left(A_{0}, A_{1}, \ldots, A_{q}\right)$ be the phase partition defined by $A_{i}=\Lambda(0,1) \cap E_{-}, A_{j}=\Lambda(0,1) \cap E_{+}$and $A_{k}=\emptyset$ for $k \neq i, j$. Obviously,

$$
\mathcal{H}^{d-1}(S) \tau^{\text {classic }}(v) \leqslant \mathcal{I}(\vec{A})=\mathcal{H}^{d-1}(S) \tau(v),
$$

whence $\tau^{\text {classic }}(v) \leqslant \tau(v)$. Conversely, let $\vec{A}=\left(A_{0}, A_{1}, \ldots, A_{q}\right)$ be a phase partition having finite surface energy. Then $A_{0}=\emptyset$, and setting $\tilde{A}_{i}=A_{i} \cup\left(E_{-} \backslash \Lambda\right)$, we have

$$
\mathcal{I}(\vec{A}) \geqslant \lim _{\varepsilon \rightarrow 0} \int_{\Lambda(0,1+\varepsilon) \cap \partial^{*} \tilde{A}_{i}} \tau\left(v_{\tilde{A}_{i}}(x)\right) \mathrm{d} \mathcal{H}^{d-1}(x) .
$$

By the convexity of the homogeneous extension of $\tau$, for any $\varepsilon>0$,

$$
\int_{\Lambda(0,1+\varepsilon) \cap \partial^{*} \tilde{A}_{i}} \tau\left(v_{\tilde{A}_{i}}(x)\right) \mathrm{d} \mathcal{H}^{d-1}(x) \geqslant|u|_{2} \tau\left(u /|u|_{2}\right),
$$

where

$$
u=\int_{\Lambda(0,1+\varepsilon) \cap \partial^{*} \tilde{A}_{i}} v_{\tilde{A}_{i}}(x) \mathrm{d} \mathcal{H}^{d-1}(x) .
$$

Since $\tilde{A}_{i} \Delta E_{-}$is included in a compact subset of the interior of $\Lambda(0,1+\varepsilon)$, then

$$
u=\int_{\Lambda(0,1+\varepsilon) \cap \partial^{*} E_{-}} v_{E_{-}}(x) \mathrm{d} \mathcal{H}^{d-1}(x)=\mathcal{H}^{d-1}\left(\Lambda(0,1+\varepsilon) \cap \partial E_{-}\right) v
$$

(see for instance [7], Proposition 3.10 for a more precise result) and therefore

$$
|u|_{2} \tau\left(u /|u|_{2}\right)=\mathcal{H}^{d-1}\left(\Lambda(0,1+\varepsilon) \cap \partial E_{-}\right) \tau(v) .
$$

Thus

$$
\mathcal{I}(\vec{A}) \geqslant \mathcal{H}^{d-1}\left(\Lambda(0,1) \cap \partial E_{-}\right) \tau(v)=\mathcal{H}^{d-1}(S) \tau(v)
$$

and taking the infimum over all phase partitions $\vec{A}$ we conclude that $\tau^{\text {classic }}(\nu) \geqslant$ $\tau(v)$. 


\section{Preliminaries}

In this section we introduce first the notation and we give some basic definitions. In the second part, we recall some useful properties of FK (or random cluster) measures and we give a short description of the Potts and Ising models and their FK representation.

\subsection{Notation}

The cardinality of a set $A$ is denoted by $|A|$. The symmetric difference between two sets $A_{1}, A_{2}$ is denoted by $A_{1} \triangle A_{2}$. For $r \in \mathbb{R},\lfloor r\rfloor$ denotes the integer part of $r$ and $\lceil r\rceil$ stands for the smallest integer larger than or equal to $r$.

Metric. We denote by $d_{p}$ the metric associated with the $p$-norm, i.e., $d_{p}(x, y)=$ $|x-y|_{p}$ for any $x, y$ in $\mathbb{R}^{d}$. We will only use the 1,2 and $\infty$ norms. The $d_{p}$ distance between two subsets $E_{1}$ and $E_{2}$ of $\mathbb{R}^{d}$ is $d_{p}\left(E_{1}, E_{2}\right)=\inf \left\{\left|x_{1}-x_{2}\right|_{p}: x_{1} \in\right.$ $\left.E_{1}, x_{2} \in E_{2}\right\}$. The $r$-neighborhood of $E \subseteq \mathbb{R}^{d}$ with respect to the $d_{p}$ metric is the set $\mathcal{V}_{p}(E, r)=\left\{x \in \mathbb{R}^{d}: d_{p}(x, E)<r\right\}$. The $d_{p}$ diameter of a subset $E$ of $\mathbb{R}^{d}$ is $\operatorname{diam}_{p} E=$ $\sup \left\{|x-y|_{p}: x, y \in E\right\}$. We will usually work with the Euclidean distance $d_{2}$ on the continuous space $\mathbb{R}^{d}$ and with the distance $d_{1}$ or $d_{\infty}$ on the discrete lattice $\mathbb{Z}^{d}$. By default, when we speak of the diameter of a set without any specification, we mean the $d_{\infty}$ diameter.

Geometric objects. Let $x=\left(x_{1}, \ldots, x_{d}\right)$ be a point of $\mathbb{R}^{d}$ and let $r$ be positive. The closed ball of center $x$ and Euclidean radius $r$ is denoted by $B(x, r)$. The sphere of center $x$ and radius $r$ is $\partial B(x, r)$. The unit sphere of $\mathbb{R}^{d}$ is denoted by $S^{d-1}$. The projective sphere $P S^{d-1}$ is obtained by identifying opposite points on $S^{d-1}$. Let $w$ be a unit vector. We set

$$
\operatorname{hyp}(x, w)=\left\{y \in \mathbb{R}^{d} ;(y-x) \cdot w=0\right\} .
$$

For $r_{1}, r_{2}$ in $\mathbb{R} \cup\{-\infty,+\infty\}$, we define

$$
\operatorname{slab}\left(x, w, r_{1}, r_{2}\right)=\left\{y \in \mathbb{R}^{d} ; r_{1} \leqslant(y-x) \cdot w \leqslant r_{2}\right\} .
$$

We set next

$$
\begin{aligned}
& B_{-}(x, r, w)=B(x, r) \cap \operatorname{slab}(x, w,-\infty, 0), \\
& B_{+}(x, r, w)=B(x, r) \cap \operatorname{slab}(x, w, 0, \infty) .
\end{aligned}
$$

By disc $(x, r, w)$ we denote the closed disc centered at $x$ of radius $r$ and normal vector $w$. A box is a set of the form

$$
\Lambda(x, r)=\left\{y=\left(y_{1}, \ldots, y_{d}\right) \in \mathbb{R}^{d} ;-r_{i} / 2<y_{i}-x_{i} \leqslant r_{i} / 2, i=1, \ldots, d\right\},
$$

where $x=\left(x_{1}, \ldots, x_{d}\right)$ and $r=\left(r_{1}, \ldots, r_{d}\right)$ belong to $\mathbb{R}^{d}$. Clearly, $x$ is the center and $r$ determines the side lengths of the box. If $r_{i}=t$ for each $i=1, \ldots, d$, where $t \in \mathbb{R}^{+}$, then we write simply $\Lambda(x, t)$. Notice that $\Lambda(x, t)$ has diameter $t$ and is neither open nor closed. If $d_{\infty}(x, y) \geqslant t$ then $\Lambda(x, t)$ and $\Lambda(y, t)$ are disjoint. Let $A$ be a subset of $\mathbb{R}^{d}$ of 
linear dimension $d-1$, that is $A$ spans a hyperplane of $\mathbb{R}^{d}$, which we denote by hyp $A$. We call such a set an hyperset. By nor $A$ we denote one of the two unit vectors orthogonal to hyp $A$, or equivalently the element of $P S^{d-1}$ orthogonal to hyp $A$. The cylinder of basis $A$ is the set

$$
\operatorname{cyl} A=\{x+t \text { nor } A ; t \in \mathbb{R}, x \in A\} .
$$

We set also $\operatorname{cyl}(A, r)=\{x+t$ nor $A:|t| \leqslant r, x \in A\}=\operatorname{cyl} A \cap \operatorname{slab}(x, \operatorname{nor} A,-r, r)$.

Topology and measure. Let $E$ be a subset of $\mathbb{R}^{d}$. We denote its interior by $\stackrel{\circ}{E}$, its closure by $\bar{E}$, its boundary by $\partial E$. Whenever $A$ is an hyperset of $\mathbb{R}^{d}$, that is $A$ spans a hyperplane of $\mathbb{R}^{d}$, we use the induced $(d-1)$-dimensional topology of hyp $A$ to define $\partial A, \AA, \bar{A}$. The collection of the Borel subsets of a set $E$ of $\mathbb{R}^{d}$ is denoted by $\mathcal{B}(E)$. The volume of a Borel set $E$ is simply its Lebesgue measure which we denote by $\mathcal{L}^{d}$. A Borel set is said to be negligible if its volume is zero. We define a (pseudo) metric dist $_{L^{1}}$ on $\mathcal{B}\left(\mathbb{R}^{d}\right)$ by

$$
\forall E, F \in \mathcal{B}\left(\mathbb{R}^{d}\right) \quad \operatorname{dist}_{L^{1}}(E, F)=\mathcal{L}^{d}(E \Delta F) .
$$

When dealing with topological questions on the space $\mathcal{B}\left(\mathbb{R}^{d}\right)$, we consider the equivalence classes of the Borel sets modulo negligible sets. We denote by $\mathcal{H}^{k}$ the standard $k$-dimensional Hausdorff measure, for $k=1,2, \ldots, d$.

The lattice $\mathbb{L}^{d}$. We turn $\mathbb{Z}^{d}$ into a graph with vertex set $\mathbb{Z}^{d}$ and edge set

$$
\mathbb{E}^{d}=\left\{\{x, y\} ; x \in \mathbb{Z}^{d}, y \in \mathbb{Z}^{d}, \mathrm{~d}_{1}(x, y)=1\right\} .
$$

This graph is called the $d$-dimensional cubic lattice and is denoted by $\mathbb{L}^{d}$. We often think of this graph as embedded in $\mathbb{R}^{d}$, the edges $\{x, y\}$ being straight line segments $[x, y]$ between nearest neighbors. If $x$ and $y$ are nearest neighbors, we denote this relation by $x \sim y$.

Let $D$ be a subset of $\mathbb{R}^{d}$. An edge $\{x, y\}$ of $\mathbb{E}^{d}$ is said to be included in $D$ if both sites $x, y$ belong to $D$. We denote by $\mathbb{E}^{d}(D)$ the set of the edges of $\mathbb{E}^{d}$ included in $D$. For $D$ a subset of $\mathbb{Z}^{d}$, the graph $\left(D, \mathbb{E}^{d}(D)\right)$ will be often identified with its vertex set $D$. For $E$ a subset of $\mathbb{E}^{d}$, a formula like $E \subset \mathbb{E}^{d}(D)$ will be abbreviated into $E \subset D$.

To simplify notation, we will sometimes identify subsets of $\mathbb{R}^{d}$ with their traces on the lattice, i.e., we identify $A \subseteq \mathbb{R}^{d}$ with $A \cap \mathbb{Z}^{d}$. For example, $\Lambda(n)$ denotes a box both in the continuum and in the lattice.

The lattice $\mathbb{L}^{d, \infty}$. We introduce another graph structure on $\mathbb{Z}^{d}$. First we define the edge set

$$
\mathbb{E}^{d, \infty}=\left\{\{x, y\} ; x \in \mathbb{Z}^{d}, y \in \mathbb{Z}^{d}, \mathrm{~d}_{\infty}(x, y)=1\right\} .
$$

The lattice $\mathbb{L}^{d, \infty}$ is defined to be the graph $\left(\mathbb{Z}^{d}, \mathbb{E}^{d, \infty}\right)$.

Discrete topology. Let $A$ be a subset of $\mathbb{Z}^{d}$. We define its edge boundary,

$$
\partial^{e d g e} A=\left\{\{x, y\} \in \mathbb{E}^{d} ; x \in A, y \in A^{c}\right\}
$$


its inner vertex boundaries,

$$
\partial^{i n} A=\left\{x \in A ; \exists y \in A^{c} y \sim x\right\}, \quad \partial_{\infty}^{i n} A=\left\{x \in A ; \exists y \in A^{c}\{x, y\} \in \mathbb{E}^{d, \infty}\right\}
$$

its outer vertex boundaries,

$$
\partial^{\text {out }} A=\left\{x \in A^{c} ; \exists y \in A y \sim x\right\}, \quad \partial_{\infty}^{\text {out }} A=\left\{x \in A^{c} ; \exists y \in A\{x, y\} \in \mathbb{E}^{d, \infty}\right\} .
$$

These definitions are extended to the subsets of $\mathbb{R}^{d}$ by setting, for $E \subset \mathbb{R}^{d}, \partial_{\times}^{*} E=$ $\partial_{\times}^{*}\left(\mathbb{Z}^{d} \cap E\right)$, where $*$ stands for edge, in or out and $\times$ stands for nothing or $\infty$.

A path $\gamma$ in $\left(\mathbb{Z}^{d}, \mathbb{E}^{d}\right)$ (respectively $\left(\mathbb{Z}^{d}, \mathbb{E}^{d, \infty}\right)$ ) is an alternating sequence $x_{0}, e_{0}, x_{1}$, $e_{1}, \ldots, e_{n-1}, x_{n}, \ldots$ of distinct vertices $x_{i}$ and edges $e_{i}$ belonging to $\left(\mathbb{Z}^{d}, \mathbb{E}^{d}\right.$ ) (respectively $\left(\mathbb{Z}^{d}, \mathbb{E}^{d, \infty}\right)$ ), where $e_{i}$ is the edge between $x_{i}$ and $x_{i+1}$. The path is said to connect every pair of its vertices. If the path terminates at some vertex $x_{n}$ it is said to have length $n$, otherwise it is infinite. Two paths are disjoint if they have no edges in common. The set $A$ is said to be connected or $\mathbb{L}^{d}$-connected (respectively $\mathbb{L}^{d, \infty}$-connected) if the graph $\left(A, \mathbb{E}^{d}(A)\right)$ (respectively $\left.\left(A, \mathbb{E}^{d, \infty}(A)\right)\right)$ is connected. Note that connectedness in the usual $\mathbb{L}^{d}$ sense implies $\mathbb{L}^{d, \infty}$-connectedness.

Let $A, B, D$ be subsets of $\mathbb{R}^{d}$. A set of edges $E \subseteq \mathbb{E}^{d}$ is said to separate $A$ and $B$ in $D$ if there is no path in the graph $\left(\mathbb{Z}^{d} \cap D, \mathbb{E}^{d}(D) \backslash E\right)$ connecting $A$ and $B$. The set $E$ separates $\infty$ in $D$ if the graph $\left(\mathbb{Z}^{d} \cap D, \mathbb{E}^{d}(D) \backslash E\right)$ has at least two infinite components.

The relevance of the lattice $\mathbb{L}^{d, \infty}$ stems from the fact that the external boundary of any $\mathbb{L}^{d, \infty}$-connected finite set $A$ in $\mathbb{Z}^{d}$ is itself $\mathbb{L}^{d, \infty}$-connected (whereas the external boundary of any $\mathbb{L}^{d}$-connected finite set in $\mathbb{Z}^{d}$ is not necessarily $\mathbb{L}^{d}$-connected). To be more specific, let us define the residual $*$-components of $A$ as the $*$-connected components of $A^{c}$ where $*$ stands for $\mathbb{L}^{d}$ or $\mathbb{L}^{d, \infty}$. Let $A$ be a $\mathbb{L}^{d, \infty}$-connected subset of $\mathbb{Z}^{d}$. If $R$ is a residual $*$-component of $A$ (in either sense), then its inner and outer vertex boundaries $\partial^{\text {in }} R$ and $\partial^{\text {out }} R$ are $\mathbb{L}^{d, \infty}$-connected (cf. [21, Lemma 2.1]) and therefore also $\partial_{\infty}^{\text {in }} R$ and $\partial_{\infty}^{\text {out }} R$ are $\mathbb{L}^{d, \infty}$-connected. We will need the notion of external boundaries. Let $A$ be a finite $\mathbb{L}^{d, \infty}$-connected set and let $R$ be its unique infinite residual $\mathbb{L}^{d}$-component. The external outer vertex boundary of $A$, denoted by $\partial^{o u t, e x t} A$, is defined as $\partial^{\text {in }} R$ and it is $\mathbb{L}^{d, \infty}$-connected. The external outer vertex $\mathbb{L}^{d, \infty}$-boundary is defined by

$$
\partial_{\infty}^{\text {out,ext }} A=\partial_{\infty}^{\text {in }} R=\left\{x \in R ; \exists y \in A \text { with }\{x, y\} \in \mathbb{E}^{d, \infty}\right\}
$$

and this set is also $\mathbb{L}^{d, \infty}$-connected.

For future reference, we prove a geometric lemma.

LEMMA 2.1. - For any finite $\mathbb{L}^{d, \infty}$-connected subset $A$ of $\mathbb{Z}^{d}$, for $r \geqslant 4$,

$$
\mathcal{L}^{d}\left(\mathcal{V}_{\infty}(A, r)\right) \leqslant 4^{d+1} r^{d-1}(|A| \vee r) .
$$

Proof. - If diam $A \leqslant r$, then $\mathcal{L}^{d}\left(\mathcal{V}_{\infty}(A, r)\right) \leqslant(3 r)^{d}$. Suppose now that $r<\operatorname{diam} A<$ $\infty$. Let $\left\{x_{1}, \ldots, x_{l}\right\}$ be a collection of vertices of $A$ of maximal cardinality such that

$$
\forall i, j \in\{1, \ldots, l\}, \quad i \neq j, \quad \Lambda\left(x_{i}, r\right) \cap \Lambda\left(x_{j}, r\right)=\emptyset .
$$


The maximality of the collection implies that $A \subset \Lambda\left(x_{1}, 2 r\right) \cup \cdots \cup \Lambda\left(x_{l}, 2 r\right)$. Because $l$ is necessarily larger or equal than 2 and $A$ is $\mathbb{L}^{d, \infty}$-connected, for each $i$ in $\{1, \ldots, l\}$,

$$
\left|A \cap \Lambda\left(x_{i}, r\right)\right| \geqslant d_{\infty}\left(x_{i}, \partial^{i n} \Lambda\left(x_{i}, r\right)\right) \geqslant r / 2-1,
$$

so that $|A| \geqslant l(r / 2-1)$. Since $\mathcal{V}_{\infty}(A, r)$ is included in $l$ boxes of diameter $4 r$, we obtain

$$
\mathcal{L}^{d}\left(\mathcal{V}_{\infty}(A, r)\right) \leqslant l(4 r)^{d} \leqslant(4 r)^{d}(r / 2-1)^{-1}|A| \leqslant 4^{d+1} r^{d-1}|A| .
$$

\subsection{FK percolation and Ising-Potts models}

Edge configurations. For $E \subseteq \mathbb{E}^{d}$ with $E \neq \emptyset$, we write $\Omega(E)$ for the set $\{0,1\}^{E}$; its elements are called edge configurations in $E$. The natural projections are given by $\operatorname{pr}_{e}: \omega \in \Omega(E) \mapsto \omega(e) \in\{0,1\}$, where $e \in E$. An edge $e$ is called open in the configuration $\omega$ if $\operatorname{pr}_{e}(\omega)=1$, and closed otherwise.

For $A \subseteq \mathbb{Z}^{d}$, let $\Omega_{A}$ stand for the set of the configurations within $A$, i.e., $\Omega_{A}=$ $\{0,1\}^{\mathbb{E}^{d}(A)}$, and $\Omega^{A}$ for the set of the configurations outside $A$, i.e., $\Omega^{A}=\{0,1\}^{\mathbb{E}^{d} \backslash \mathbb{E}^{d}(A)}$. (Recall that $\mathbb{E}^{d}(A)$ denotes the set of edges between sites in $A$.) In general, for $A \subseteq$ $B \subseteq \mathbb{Z}^{d}$, we set $\Omega_{B}^{A}=\{0,1\}^{\mathbb{E}^{d}(B) \backslash \mathbb{E}^{d}(A)}$. Given $\omega \in \Omega$ and $E \subseteq \mathbb{E}^{d}$, we denote by $\omega(E)$ the restriction of $\omega$ to $\Omega(E)$. Analogously, $\omega_{B}^{A}$ stands for the restriction of $\omega$ to the set $\mathbb{E}^{d}(B) \backslash \mathbb{E}^{d}(A)$.

Given $\eta \in \Omega$, we denote by $\mathcal{O}(\eta)$ the set of the edges of $\mathbb{E}^{d}$ which are open in the configuration $\eta$. The connected components of the graph $\left(\mathbb{Z}^{d}, \mathcal{O}(\eta)\right)$ are called $\eta$ clusters. The path $\gamma=\left(x_{1}, e_{1}, x_{2}, \ldots\right)$ is said to be $\eta$-open if all the edges $e_{i}$ belong to $\mathcal{O}(\eta)$. We write $\{A \leftrightarrow B\}$ for the event that there exists an open path joining some site in $A$ with some site in $B$. Similarly, we denote by $\{A \leftrightarrow \infty\}$ the event that there exists $x \in A$ lying in an infinite component.

Let $\omega$ be an edge configuration in $\mathbb{Z}^{d}$ (or in a subgraph of $\mathbb{L}^{d}$ ). We can look at the open clusters in $V$ or alternatively the open $V$-clusters. These clusters are simply the connected components of the random graph $(V, \mathcal{O}(\omega(E)))$, where $\omega(E)$ is the restriction of $\omega$ to $E$.

Given $E \subseteq \mathbb{E}^{d}$, we write $\mathcal{F}(E)$ for the $\sigma$-field generated by the finite-dimensional cylinders associated with configurations in $\Omega(E)$. Similarly, for $A \subseteq B \subseteq \mathbb{Z}^{d}$, we use the notation $\mathcal{F}_{B}^{A}$ for the $\sigma$-field generated by finite-dimensional cylinders associated with configurations in $\Omega_{B}^{A}$. If $A=\emptyset$ or $B=\mathbb{Z}^{d}$, then we omit them from the notation.

Stochastic domination. There is a partial order $\preceq$ in $\Omega$ given by $\omega \preceq \omega^{\prime}$ iff $\omega(e) \leqslant$ $\omega^{\prime}(e)$ for every $e \in \mathbb{E}^{d}$. A function $f: \Omega \rightarrow \mathbb{R}$ is called increasing if $f(\omega) \leqslant f\left(\omega^{\prime}\right)$ whenever $\omega \preceq \omega^{\prime}$. An event is called increasing if its characteristic function is increasing. Let $\mathcal{F}$ be a $\sigma$-field of subsets of $\Omega$. For a pair of probability measures $\mu$ and $\nu$ on $(\Omega, \mathcal{F})$, we say that $\mu$ (stochastically) dominates $v$ if for any $\mathcal{F}$-measurable increasing function $f$ the expectations satisfy $\mu(f) \geqslant v(f)$. If, in addition, for each $\mathcal{F}$-measurable cylinder $Z$ with $\mu(Z) \wedge v(Z)>0$, we have $\mu(f \mid Z) \geqslant v(f \mid Z)$, then we say that $\mu$ strongly dominates $v$, and we denote this relation by $\mu \succeq v$.

FK measures. Let $V \subseteq \mathbb{Z}^{d}$ be finite and $E=\mathbb{E}^{d}(V)$. We first introduce (partially wired) boundary conditions as follows. Consider a partition $\pi$ of the set $\partial^{i n} V$, say $\pi=$ 
$\left\{B_{1}, \ldots, B_{n}\right\}$. (The sets $B_{i}$ are disjoint non-empty subsets of $\partial^{i n} V$ with $B_{1} \cup \cdots \cup B_{n}=$ $\left.\partial^{i n} V\right)$. We say that $x, y \in \partial^{i n} V$ are $\pi$-connected, if $x, y \in B_{i}$ for an $i \in\{1, \ldots, n\}$. Fix a configuration $\eta \in \Omega_{V}$. We introduce an equivalence relation on $V: x$ and $y$ are said to be $\pi \cdot \eta$-connected if they are both joined by $\eta$-open paths to (or identical with) sites $x^{\prime}, y^{\prime} \in \partial^{i n} V$ which are themselves $\pi$-wired. The new equivalence classes are called $\pi \cdot \eta$-clusters, or $F K$ clusters in $V$ with respect to the boundary condition $\pi$. The number of FK clusters (w.r.t. $\pi$ ) is denoted by $c l^{\pi}(\eta)$. In general, we will use $C$ to denote clusters and $C^{\mathrm{FK}}$ for FK clusters.

For fixed $p \in[0,1]$ and $q \geqslant 1$, the $F K$ measure with parameters $(p, q)$ and boundary conditions $\pi$ is a probability measure on the $\sigma$-field $\mathcal{F}_{V}$, defined by the formula

$$
\forall \eta \in \Omega_{V} \quad \Phi_{V}^{\pi, p, q}[\{\eta\}]=\frac{1}{Z_{V}^{\pi, p, q}}\left(\prod_{e \in E} p^{\eta(e)}(1-p)^{1-\eta(e)}\right) q^{c l^{\pi}(\eta)},
$$

where $Z_{V}^{\pi, p, q}$ is the appropriate normalization factor. Since $\mathcal{F}_{V}$ is an atomic $\sigma$-field with atoms $\{\eta\}, \eta \in \Omega_{V}$, (6) determines a unique measure on $\mathcal{F}_{V}$. Note that every cylinder has non-zero probability. There are two extremal b.c.s: the free boundary condition corresponds to the partition $f$ defined to have exactly $\left|\partial^{\text {in }} V\right|$ classes, and the wired b.c. corresponds to the partition $w$ with only one class. The set of all such measures called FK (or random cluster) measures corresponding to different b.c.s will be denoted by $\mathcal{F} \mathcal{K}(p, q, V)$, and we write $c \mathcal{F} \mathcal{K}(p, q, V)$ for its convex hull. The stochastic process $\left(\operatorname{pr}_{e}\right)_{e \in \mathbb{E}^{d}(V)}: \Omega \rightarrow \Omega_{V}$ given on the probability space $\left(\Omega, \mathcal{F}, \Phi_{V}^{\pi, p, q}\right)$ is called FK percolation with boundary conditions $\pi$.

We will list some useful properties of FK measures. A property of crucial importance is that for $q \geqslant 1$, every $\Phi \in \mathcal{F} \mathcal{K}(p, q, V)$ is strong $F K G$. This means that for every $\mathcal{F}_{V}$-measurable cylinder $Z$, and for all $\mathcal{F}_{V}$-measurable increasing functions $f$, $g$, we have

$$
\Phi[f g \mid Z] \geqslant \Phi[f \mid Z] \Phi[g \mid Z] .
$$

In some cases it is possible to compare FK measures with different b.c.s. There is a partial order on the set of partitions of $\partial^{i n} V$. We say that $\pi$ dominates $\pi^{\prime}, \pi \geqslant \pi^{\prime}$, if $x, y \pi^{\prime}$-wired implies that they are $\pi$-wired. We then have $\Phi_{V}^{\pi^{\prime}, p, q} \preceq \Phi_{V}^{\pi, p, q}$. This implies immediately that for each $\Phi \in \mathcal{F} \mathcal{K}(p, q, V), \Phi_{V}^{f, p, q} \preceq \Phi \preceq \Phi_{V}^{\bar{w}, p, q}$. Next we discuss properties of conditional FK measures. For given $U \subseteq V$ and $\omega \in \Omega$, we define a partition $W_{V}^{U}(\omega)$ of $\partial^{i n} U$ by declaring $x, y \in \partial^{i n} U$ to be $W_{V}^{U}(\omega)$-wired if they are joined by an $\omega_{V}^{U}$-open path. Fix a partition $\pi$ of $\partial^{i n} V$. We define a new partition of $\partial^{i n} U$, denoted by $\pi \cdot W_{V}^{U}(\omega)$, by considering $x, y \in \partial^{i n} U$ to be $\pi \cdot W_{V}^{U}(\omega)$-wired if they are both joined by $\omega_{V}^{U}$-open paths to (or identical with) sites $x^{\prime}, y^{\prime}$, which are themselves $\pi$-wired. Then, for every $\mathcal{F}_{U}$-measurable function $f$,

$$
\Phi_{V}^{\pi, p, q}\left[f \mid \mathcal{F}_{V}^{U}\right](\omega)=\Phi_{U}^{\pi \cdot W_{V}^{U}(\omega), p, q}[f], \quad \Phi_{V}^{\pi, p, q} \text {-a.s. }
$$

Note that (8) can be interpreted as a kind of Markov property. A direct consequence of this formula is that the restriction of every FK measure $\Phi$ in $\mathcal{F} \mathcal{K}(p, q, V)$ to $\mathcal{F}_{U}$ is contained in the convex hull $c \mathcal{F} \mathcal{K}(p, q, U)$. 
Ising-Potts measure. Let $V \subset \mathbb{Z}^{d}$ be finite and let $q \geqslant 2$ be an integer. A spin (color) configuration in $V$ is a map $\sigma: V \rightarrow\{1,2, \ldots, q\}$. We denote by $\sigma(x)$ the spin at site $x$ in the configuration $\sigma$. To define (mixed) boundary conditions (b.c.s) on $V$ we consider an ordered partition $\rho$ of $\partial^{\text {in }} V$ into $q+1$ disjoint sets $\left(R^{0}, R^{1}, \ldots, R^{q}\right)$. A configuration $\sigma$ is said to be $\rho$-compatible if $\sigma_{\left\lceil R^{i}\right.}=i$ for $i=1, \ldots, q$. Note that there is no constraint on the spins in $R^{0}$. The constant b.c.s with $R^{i}=\partial^{i n} V$ for some $i$ are called $i$-b.c.s for $i=1, \ldots, q$ and free-b.c.s for $i=0$.

Let $\rho$ be fixed. The energy or Hamiltonian of a configuration is given by

$$
H_{V}^{\rho}(\sigma)= \begin{cases}\sum_{\{x, y\}, x \sim y} 1_{\sigma(x) \neq \sigma(y)} ; & \text { if } \sigma \text { is } \rho \text {-compatible, } \\ \infty ; & \text { otherwise. }\end{cases}
$$

For $\beta>0$, the corresponding Gibbs measure $\mu_{V}^{\beta, q, \rho}$ with boundary conditions $\rho$ at inverse temperature $\beta$ is the probability measure on $\{1, \ldots, q\}^{V}$ defined by:

$$
\mu_{V}^{\beta, q, \rho}[\sigma]=\exp \left(-\beta H_{V}^{\rho}(\sigma)\right) / Z(\beta, q, \rho, V),
$$

where $Z(\beta, q, \rho, V)$ is the normalizing factor, called the partition function, given by

$$
Z(\beta, q, \rho, V)=\sum_{\sigma \in\{1, \ldots, q\}^{V}} \exp \left(-\beta H_{V}^{\rho}(\sigma)\right) .
$$

FK representation of Ising-Potts measures. We describe a coupling, constructed by Fortuin and Kasteleyn [27], between FK percolation and the Ising-Potts model (see e.g. [45] and the references therein for more details). Let $V \subset \mathbb{Z}^{d}$ be finite. An edge-spin configuration in $V$ is an element $(\omega, \sigma)$ of $\{0,1\}^{\mathbb{E}^{d}(V)} \times\{1, \ldots, q\}^{V}$. Let $\rho=\left(R^{0}, R^{1}, \ldots, R^{q}\right)$ be a b.c. for the spin model and let $\rho^{\prime}$ be the boundary condition for the percolation model defined as follows: the equivalence classes of the partition $\rho^{\prime}$ are $R^{1}, \ldots, R^{q}$ plus all the singletons $\{x\}, x \in R^{0}$. In words: wired b.c.s on the $R^{i}$-s for $i=1, \ldots, q$ (but these sets are not wired together) and free b.c.s on $R^{0}$. We denote by $\Phi_{V}^{p, q, \rho^{\prime}, \leftrightarrow}$ the probability measure on the edge configurations in $V$ obtained by conditioning the regular FK measure $\Phi_{V}^{p, q, \rho^{\prime}}$ on not having any open connections between the sets $R^{i}, R^{j}$ for $1 \leqslant i<j \leqslant q$. Note that in the case of constant b.c.s there is no restriction on the existence of open connections.

For a given $\beta>0$, we set $p=1-\mathrm{e}^{-\beta}$. We can sample a spin configuration from the distribution $\mu_{V}^{\beta, q, \rho}$ as follows. First we draw an edge configuration in $V$ according to $\Phi_{V}^{p, q, \rho^{\prime}, \triangleleft}$. In a second step we color each open cluster independently, with color (spin) $i$ for clusters intersecting $R^{i}$ and with the uniform distribution on $\{1, \ldots, q\}$ for the other clusters. In this way we obtain a random edge-spin configuration whose distribution will be denoted by $\mathbb{P}_{V}^{\beta, q, \rho}$. We refer to a process of edge-spin configurations with this law as the FK coupling of the Ising-Potts and FK percolation processes.

Basic asymptotic properties of FK and Ising-Potts measures. It is known that for $*=f$ or $w$, the weak limits $\Phi_{\infty}^{p, q, *}=\lim _{n \rightarrow \infty} \Phi_{\Lambda(n)}^{p, q, *}$ exist and are translation invariant. Moreover they are extremal w.r.t. stochastic ordering among measures obtained as weak 
limits of FK measures with mixed boundary conditions. The order parameter of FK percolation is given by the percolation probability $\theta^{w}=\theta^{w}(p, q, d)=\Phi_{\infty}^{p, q, w}[0 \leftrightarrow \infty]$. It is known that for $d \geqslant 2$ the system exhibits a phase transition, more precisely, there exists $p_{c}=p_{c}(q, d) \in(0,1)$ such that $\theta^{w}(p)=0$ for $p<p_{c}$ and $\theta^{w}(p)>0$ for $p>p_{c}$. It has been conjectured that $\Phi_{\infty}^{p, q, w}=\Phi_{\infty}^{p, q, f}$ (which is known to be equivalent to $\left.\theta^{w}(p)=\theta^{f}(p)\right)$ when $p \neq p_{c}$ but it is only known that the complement of the set of regular points

$$
\mathcal{U}(q, d)=\left\{p \in[0,1] ; \Phi_{\infty}^{p, q, w}=\Phi_{\infty}^{p, q, f}\right\}
$$

can have at most countably many elements. More about this topic can be found in [30].

Assume $p \in \mathcal{U}(q, d)$ and $p>\hat{p}_{c}$. Typical configurations (w.r.t. any measure in $c \mathcal{F} \mathcal{K}(p, q, \Lambda))$ in a large finite box $\Lambda$ have the following properties: there exists a unique largest cluster which is "omnipresent", in particular, it crosses the box from wall to wall in each direction. Its density is close to $\theta$ and its mass is homogeneously distributed in the entire box. Most of the remaining clusters are bounded in diameter by a constant $L=L(p, q, d)$. More precisely, these latter clusters and the largest cluster fill up the box up to a negligible fractional volume $\varepsilon$. Large deviations estimates for (the complements) of such events can be found in [49]. By using the FK representation of the corresponding spin models it is easy to derive the following information about the "pure phases" of Ising-Potts models: in a large box with constant 1-boundary conditions (or if we restrict the infinite volume measure $\mu_{\infty}^{\beta, q,(1)}$ to the box), we typically see a large spin cluster of color 1 which is omnipresent in the box. All the different colors (spins) are homogeneously distributed in the box and they have densities $\theta+(1-\theta) / q$ for spin $i=1$, and $(1-\theta) / q$ for $i=2, \ldots, q$.

The region $\Omega$. We consider a bounded, open and connected region $\Omega$ in $\mathbb{R}^{d}$ with boundary $\Gamma=\partial \Omega$ satisfying the following assumption:

Hypothesis on $\Omega$. - We suppose that $\Omega$ is a Lipschitz domain, i.e., its boundary $\Gamma$ can be locally represented as the graph of a Lipschitz function defined on some open ball of $\mathbb{R}^{d-1}$.

The precise condition can be expressed as follows: each point $x$ of $\Gamma=\partial \Omega$ has a neighborhood $U$ such that $U \cap \Omega$ is represented by the inequality $x_{n}<f\left(x_{1}, \ldots, x_{n-1}\right)$ in some cartesian coordinate system where $f$ is a function satisfying a Lipschitz condition. Such domains are usually called Lipschitz domains in the literature and they possess all the geometric properties we will need in the course of our proofs. In particular the boundary $\Gamma$ of $\Omega$ is $d-1$ rectifiable (in the terminology of Federer's book [26]), so that its Minkowski content is equal to $\mathcal{H}^{d-1}(\Gamma)$. In addition, a Lipschitz domain $\Omega$ is admissible (in the terminology of Ziemer's book [54]) and in particular $\mathcal{H}^{d-1}\left(\Gamma \backslash \partial^{*} \Omega\right)=$ 0 . Moreover, each point of $\Gamma$ is accessible from $\Omega$ through a rectifiable arc.

Note that this hypothesis is automatically satisfied when $\Omega$ is a bounded open set with a $C^{1}$ boundary or when $\Omega$ is a polyhedral domain.

We will study Ising-Potts models and FK percolation on discretized versions of $\Omega$. More precisely, we define for $n \in \mathbb{N}$,

$$
\mathbb{Z}_{n}^{d}=\mathbb{Z}^{d} / n \quad \text { (the rescaled lattice), }
$$




$$
\begin{aligned}
& \left.\Omega_{n}=\left\{x \in \mathbb{Z}_{n}^{d} ; \mathrm{d}_{\infty}(x, \Omega)<1 / n\right\} \quad \text { (the discrete counterpart of } \Omega\right), \\
& \Gamma_{n}=\partial^{i n} \Omega_{n} \quad\left(\text { the inner vertex boundary of } \Omega_{n}\right) .
\end{aligned}
$$

\section{Coarse graining of FK processes on $\Omega_{n}$.}

The blocks and the block events. Let $n$ and $K$ be positive integers whose value will be fixed for the sequel. For $\underline{x} \in \mathbb{Z}^{d}$, we define the block indexed by $\underline{x}$ as $B(\underline{x})=$ $\Lambda(\underline{x} K / n, K / n)$. Note that the blocks partition the entire space, in particular $\mathbb{Z}_{n}^{d}$. We will sometimes identify the block $B(\underline{x})$ with its index $\underline{x}$. In particular, we will speak about nearest neighbor blocks, $\mathbb{L}^{d}$ - or $\mathbb{L}^{d, \infty}$-connected components of blocks, and about the various boundaries of such sets. If $\underline{A}$ is a subset of $\mathbb{Z}^{d}$, we define $B(\underline{A})$ to be the union of the blocks indexed by $\underline{A}$, i.e.,

$$
B(\underline{A})=\bigcup_{\underline{x} \in \underline{A}} B(\underline{x}) .
$$

To obtain a coarse graining of FK percolation in $\Omega_{n}$ we will consider events which can be observed within the individual blocks or in their neighborhood. Let $\alpha$ be a positive integer, called the event-block size. For $\underline{x} \in \mathbb{Z}^{d}$, we introduce a larger block $B^{\prime}(\underline{x})$ around $B(\underline{x})$, called the event-block, by setting

$$
B^{\prime}(\underline{x})=\bigcup_{\underline{z} ; \mathrm{d}_{\infty}(\underline{z}, \underline{x})<\alpha} B(\underline{z}) .
$$

Note that $\alpha$ is equal to the total number of layers of boxes in $B^{\prime}(\underline{x})$.

LEMMA 2.2. - Under our hypothesis on $\Omega$, there exists an increasing sequence of open and connected sets $\Omega_{n}^{\prime}$ with $\bigcup_{n \in \mathbb{N}} \Omega_{n}^{\prime}=\Omega$ such that

$$
\begin{aligned}
\sup _{n \in \mathbb{N}} \mathcal{H}^{d-1}\left(\partial \Omega_{n}^{\prime}\right) & <\infty, \\
\lim _{n \rightarrow \infty} \frac{n}{f(n)} \mathrm{d}_{2}\left(\partial \Omega_{n}^{\prime}, \Gamma\right) & =\infty,
\end{aligned}
$$

where $f$ is our fixed function satisfying (1).

Remark. - A stronger statement is proved in [42], namely, an approximating sequence of strict subsets of $\Omega$ is built whose perimeter converges to the perimeter of $\Omega$.

Proof. - For $t>0$, let us define

$$
\Omega(t)=\left\{x \in \Omega: d_{2}(x, \Gamma)>t\right\} .
$$

Let $f: \Omega \rightarrow \mathbb{R}^{+}$be the map defined by $f(x)=d_{2}(x, \Gamma)$. This map is Lipschitzian with Lipschitz constant 1 . We apply the coarea formula to $f$ and $\Omega \backslash \Omega(1 / m)$ (see [26, Theorem 3.2.11], or [24, paragraph 3.4.2]). We have for $m \in \mathbb{N}$

$$
\begin{aligned}
\mathcal{L}^{d}(\Omega \backslash \Omega(1 / m)) & \geqslant \int_{\Omega \backslash \Omega(1 / m)}|\nabla f(x)| \mathrm{d} \mathcal{L}^{d}(x) \\
& \geqslant \int_{\mathbb{R}} \mathcal{H}^{d-1}\left((\Omega \backslash \Omega(1 / m)) \cap f^{-1}(t)\right) \mathrm{d} t=\int_{0}^{1 / m} \mathcal{H}^{d-1}(\partial \Omega(t)) \mathrm{d} t
\end{aligned}
$$


hence there exists $t(m)$ in $(0,1 / m)$ such that

$$
m \mathcal{L}^{d}(\Omega \backslash \Omega(t(m))) \geqslant \mathcal{H}^{d-1}(\partial \Omega(t(m))) .
$$

Our hypothesis on $\Omega$ implies in particular that the boundary $\Gamma$ of $\Omega$ is $d-1$ rectifiable (in the terminology of Federer's book [26]) and closed, therefore its Minkowski content is equal to $\mathcal{H}^{d-1}(\Gamma)$ (see the appendix for details), hence

$$
\lim _{m \rightarrow \infty} \frac{m}{2} \mathcal{L}^{d}\left(\left\{x \in \mathbb{R}^{d}: d_{2}(x, \Gamma)<1 / m\right\}\right)=\mathcal{H}^{d-1}(\Gamma) \geqslant \frac{1}{2} \limsup _{m \rightarrow \infty} \mathcal{H}^{d-1}(\partial \Omega(t(m))) .
$$

Notice that the set $\Omega(t(m))$ is not necessarily connected. Let us fix a point $x_{0}$ in $\Omega$. Let $\Omega\left(t(m), x_{0}\right)$ be the connected component of $\Omega(t(m))$ containing $x_{0}$. Obviously $\mathcal{H}^{d-1}(\partial \Omega(t(m))) \geqslant \mathcal{H}^{d-1}\left(\partial \Omega\left(t(m), x_{0}\right)\right)$ and therefore

$$
\limsup _{m \rightarrow \infty} \mathcal{H}^{d-1}\left(\partial \Omega\left(t(m), x_{0}\right)\right) \leqslant 2 \mathcal{H}^{d-1}(\Gamma)
$$

so that (10) is satisfied. Since $\lim _{m \rightarrow \infty} t(m)=0$, we can extract from $(t(m))_{m \in \mathbb{N}}$ a decreasing subsequence $(u(m))_{m \in \mathbb{N}}$. The sequence of sets $\left(\Omega\left(u(m), x_{0}\right)\right)_{m \in \mathbb{N}}$ is then increasing, and since $\Omega$ is connected, we have

$$
\bigcup_{m \in \mathbb{N}} \Omega\left(u(m), x_{0}\right)=\Omega
$$

Finally, the sequence $\left(d_{2}\left(\Omega\left(u(m), x_{0}\right), \Gamma\right)\right)_{m \in \mathbb{N}}$ is positive and decreases to 0 . By reindexing appropriately our sequence of sets, we can ensure condition (11).

It turns out, somewhat surprisingly, that it will be sufficient to have a coarse grained picture of the FK process in a certain neighborhood of the set $\Omega_{n}^{\prime}$. For given $n, \alpha, K$ (whose value may depend on $n$ ) and function $f: \mathbb{N} \rightarrow \mathbb{N}$ satisfying (1) we consider the following collection of blocks:

$$
\underline{\Omega}_{n}=\left\{\underline{x} \in \mathbb{Z}^{d} ; \mathrm{d}_{2}\left(\underline{x} K / n, \Omega_{n}^{\prime}\right)<\mathrm{d}_{2}\left(\partial \Omega_{n}^{\prime}, \Gamma\right) / 2 \text { and } \alpha K / n<\mathrm{d}_{2}(\underline{x} K / n, \Gamma)\right\} .
$$

Note that the event-blocks cover the $f(n)$-neighborhood of $\Omega_{n}^{\prime}$ and are entirely contained in $\Omega_{n}$.

Block events. Let $\Lambda$ be a box in $\mathbb{Z}^{d}$ with side-length equal to $k$. An open cluster within $\Lambda$ is called crossing for $\Lambda$ if it intersects each of the $2 d$ faces of $\partial^{i n} \Lambda$. Let $g$ be an increasing function from $\mathbb{N}$ to $\mathbb{R}^{+}$such that $g(k) \leqslant k$ for all $k$ and let $\delta>0$. We consider the following events:

$U(\Lambda)=\left\{\right.$ there exists a unique open crossing cluster $C^{*}$ in $\left.\Lambda\right\}$,

$R(\Lambda, g)=U(\Lambda) \cap\{\exists$ ! open cluster with diameter $\geqslant g(k)\}$,

$O(\Lambda, g)=R(\Lambda, g) \cap\left\{C^{*}\right.$ intersects every sub-box of $\Lambda$ of diameter $\left.\geqslant g(k)\right\}$,

$V(\Lambda, \delta)=U(\Lambda) \cap\left\{(\theta-\delta)|\Lambda|<\left|C^{*}\right|<(\theta+\delta)|\Lambda|\right\}$, 


$$
\begin{array}{r}
T(\Lambda, g, \delta)=O(\Lambda, g) \cap\left\{\text { for any box } \Lambda^{\prime} \subset \Lambda \text { of diameter } \geqslant g(k),\right. \\
\left.(\theta-\delta)\left|\Lambda^{\prime}\right|<\left|C^{*} \cap \Lambda^{\prime}\right|<(\theta+\delta)\left|\Lambda^{\prime}\right|\right\} .
\end{array}
$$

Theorem 3.1 in [49] implies that for $d \geqslant 3, q \geqslant 1, p>\widehat{p}_{c}, \theta^{f}(p)=\theta^{w}(p)$, there exist positive constants $b=b(p, q, d), c=c(p, q, d)$ and $\kappa=\kappa(p, q, d)$, such that for each $k \geqslant 1$, each box with side-length $k$, and each measure $\Phi \in c \mathcal{F} \mathcal{K}(p, q, \Lambda)$

$$
\Phi\left[U(\Lambda)^{c}\right] \leqslant b \exp (-c k) .
$$

Moreover, if $\kappa \ln k \leqslant g(k) \leqslant k$ for all $k$ in $\mathbb{N}$,

$$
\Phi\left[R(\Lambda, g)^{c}\right] \leqslant \Phi\left[O(\Lambda, g)^{c}\right] \leqslant b \exp (-c g(k)) .
$$

Also, for $\delta>0$, there exist positive constants $b=b(p, q, d, \delta), c=c(p, q, d, \delta)$ and $\kappa=\kappa(p, q, d, \delta)$, such that for each $k \geqslant 1$, each box with side-length $k$, and each measure $\Phi \in c \mathcal{F} \mathcal{K}(p, q, \Lambda)$

$$
\Phi\left[V(\Lambda, \delta)^{c}\right] \leqslant b \exp (-c k)
$$

and, if $\kappa \ln k \leqslant g(k) \leqslant k$ for all $k$ in $\mathbb{N}$,

$$
\Phi\left[T(\Lambda, g, \delta)^{c}\right] \leqslant b \exp (-c g(k)) .
$$

Notice that we have introduced a new type of event named $T(\Lambda, g, \delta)$. The corresponding estimate follows from Theorems 3.1 and 1.2 in [49].

We will have to work on the lattices $\mathbb{Z}_{n}^{d}$ for $n \geqslant 1$. In order to keep the notation relatively simple we adopt the following convention. When working on the lattice $\mathbb{Z}_{n}^{d}$ with $n \neq 1$ the events described in (12) have to be understood in scales adapted to the actual lattice spacing. In particular, the effective diameter of $\Lambda$ with "side length $k$ " will be $k / n$, a sub-box of "diameter $\geqslant g(k)$ " will have diameter $\geqslant g(k) / n$ etc. In general, length and volume have to be measured on the actual lattice scale. This is of course not valid for the cardinality of sets.

Block variables. In the course of the proofs we will often use coarse graining in $\underline{\Omega}_{n}$ by looking at a block process $(X(\underline{x}))_{\underline{x} \in \underline{\Omega}_{n}}$, indicating the non-occurrence of one of the typical events listed in (12) in the corresponding event-block. (According to our convention, the block size has to be measured in the lattice units!) By controlling the coarse grained process $X$ we can extract useful information about the underlying FK process; in fact this is our main tool to control the microscopic behavior of the model. The definition of the events and the estimates (13)-(16) guarantee that the block process satisfies the following properties:

$$
\begin{aligned}
& \text { - the variable } X(\underline{x}) \text { depends only on the edges in } B^{\prime}(\underline{x}), \\
& \text { - } \max _{\Phi \in c \mathcal{F} \mathcal{K}\left(p, q, B^{\prime}(\underline{x})\right)} \Phi[X(\underline{x})=1] \leqslant \varepsilon .
\end{aligned}
$$

These two properties imply furthermore

$$
\max _{\Phi \in c \mathcal{F} \mathcal{K}\left(p, q, \Omega_{n}\right)} \Phi\left[X(\underline{x})=1 \mid \sigma\left(X(\underline{z}), \mathrm{d}_{\infty}(\underline{x}, \underline{z}) \geqslant 2 \alpha-1\right)\right] \leqslant \varepsilon .
$$

For later reference we re-state Lemma 2.2 from [15]. 
LEMma 2.3. - Consider a $0-1$ valued random field $\left(X_{z}\right)_{z \in \Lambda(m)}$ with the property that there exist a positive integer $D$ and $\varepsilon \in[0,1]$ such that for each $z \in \Lambda(m)$,

$$
P\left[X_{z}=1 \mid \sigma\left(X_{y} ; \mathrm{d}_{\infty}(z, y) \geqslant D\right)\right] \leqslant \varepsilon .
$$

Then, for every $\delta \in(\varepsilon, 1]$,

$$
P\left[\frac{1}{m^{d}} \sum_{z \in \Lambda(m)} X_{z} \geqslant \delta\right] \leqslant D^{d} \exp \left(-\Lambda_{\varepsilon}^{*}(\delta)\left\lfloor\frac{m}{D}\right\rfloor^{d}\right),
$$

where

$$
\Lambda_{\varepsilon}^{*}(\delta)=\delta \log \frac{\delta}{\varepsilon}+(1-\delta) \log \frac{1-\delta}{1-\varepsilon}
$$

is the Legendre transform of the logarithmic moment generating function of a Bernoulli variable with parameter $\varepsilon$. (We remark that if $\varepsilon<\delta \leqslant 1 / 2$, then $\Lambda_{\varepsilon}^{*}(\delta) \geqslant \delta \log (\delta / \varepsilon)-$ $\log 2$.

\section{The surface energy of partitions}

In this section, we introduce the metric on the space of partitions with finite perimeter and we prove the basic geometrical results necessary to obtain the large deviations principles. Some extra technical work is needed compared to the existing results because we wish to take into account boundary effects. Apart from this additional feature, the metric on the space of partitions Dist is the one used by Congedo and Tamanini [16-18]; for a careful exposition and study of this metric on the space of Caccioppoli partitions, see [40].

Throughout the section, we consider an open bounded domain $\Omega$ in $\mathbb{R}^{d}$, together with a sequence $\left(\Gamma^{m}\right)_{m \in \mathbb{N}}$ of (possibly empty) disjoint subsets of its boundary $\Gamma=\partial \Omega$.

Hypothesis. - We suppose that the boundary $\Gamma$ of $\Omega$ can be locally represented as the graph of a Lipschitz function defined on some open ball of $\mathbb{R}^{d-1}$. For each $m$ in $\mathbb{N}$, the set $\Gamma^{m}$ is open for the relative topology of $\Gamma$. The relative boundary of $\Gamma \backslash \bigcup_{m} \Gamma^{m}$ in $\Gamma$ has null $\mathcal{H}^{d-1}$ measure.

The precise condition on $\Omega$ can be expressed as follows: each point $x$ of $\Gamma=\partial \Omega$ has a neighborhood $U$ such that $U \cap \Omega$ is represented by the inequality $x_{d}<f\left(x_{1}, \ldots, x_{d-1}\right)$ in some cartesian coordinate system where $f$ is a function satisfying a Lipschitz condition. Such domains are usually called Lipschitz domains in the literature and they possess all the geometric properties we will need in the course of our proofs. First the boundary $\Gamma$ of $\Omega$ is $d-1$ rectifiable (in the terminology of Federer's book [26]), so that its Minkowski content is equal to $\mathcal{H}^{d-1}(\Gamma)$. Second, a Lipschitz domain $\Omega$ is admissible (in the terminology of Ziemer's book [54]) and therefore $\mathcal{H}^{d-1}\left(\Gamma \backslash \partial^{*} \Omega\right)=0$. Third, each point of $\Gamma$ is accessible from $\Omega$ through a rectifiable arc.

We recall that the relative topology of $\Gamma$ is the topology induced on $\Gamma$ by the topology of $\mathbb{R}^{d}$. Hence each set $\Gamma^{m}$ is the intersection of $\Gamma$ with an open set of $\mathbb{R}^{d}$. Finally the last sentence in the hypothesis is equivalent to saying $\mathcal{H}^{d-1}\left(\partial_{\Gamma} \bigcup_{m} \Gamma^{m}\right)=0$, where $\partial_{\Gamma}$ is 
the boundary for the topology induced by $\mathbb{R}^{d}$ on $\Gamma$. Since the sets $\Gamma^{m}$ are relatively open and disjoint, we have $\bigcup_{m} \partial_{\Gamma} \Gamma^{m} \subset \partial_{\Gamma} \bigcup_{m} \Gamma^{m}$.

The sequence $\left(\Gamma^{m}\right)_{m \in \mathbb{N}}$ induces the b.c. on $\Omega$ in the following way: for any $m \in \mathbb{N}$, the points belonging to $\Gamma^{m}$ are wired together, while the points in $\Gamma \backslash \bigcup_{m} \Gamma^{m}$ are let free. The aim of this section is to describe the geometric macroscopic object which emerges from the FK measure defined inside $\Omega$ with b.c. induced by the sequence $\left(\Gamma^{m}\right)_{m \in \mathbb{N}}$.

A partition $\mathcal{A}$ of $\Omega$ is a finite or countable collection of non-negligible Borel subsets of $\Omega$ which, up to negligible sets, form a partition of $\Omega$. The perimeter $\mathcal{P}(\mathcal{A})$ of a partition $\mathcal{A}$ is defined as

$$
\mathcal{P}(\mathcal{A})=\sum_{A \in \mathcal{A}} \mathcal{P}(A)
$$

A set of a partition is the macroscopic object corresponding to a large $F K$ cluster of the percolation configuration. Because we wish to take into account the effect of b.c., we need to keep track of the connections between the clusters and the boundary. Unfortunately, the macroscopic picture of the sets alone does not describe what might happen on the microscopic level near $\Gamma$. Hence we record separately the relevant information with a touching function.

A touching function associated with a partition $\mathcal{A}$ of $\Omega$ is a map $\mathcal{T}: \mathcal{A} \times \mathbb{N} \mapsto\{0,1\}$ describing the contacts between the sets of $\mathcal{A}$ and the boundary pieces $\left(\Gamma^{m}\right)_{m \in \mathbb{N}}$. A touching function must satisfy the following compatibility condition: a boundary piece $\Gamma^{m}$ can touch at most one set of the partition, or equivalently,

$$
\forall m \in \mathbb{N} \quad \sum_{A \in \mathcal{A}} \mathcal{T}(A, m) \leqslant 1
$$

Indeed, a set of $\mathcal{A}$ is the macroscopic object corresponding to a large FK cluster of the percolation configuration, and to define the FK clusters we take into account the boundary conditions.

We say that a partition $\mathcal{A}$ of $\Omega$ has touching status $\mathcal{T}$ (or that $\mathcal{A}$ is a partition of $\Omega$ with touching status $\mathcal{T}$ ) if $\mathcal{T}$ is a touching function associated with $\mathcal{A}$. Formally, a partition with touching status is a pair $(\mathcal{A}, \mathcal{T})$ where $\mathcal{A}$ is a partition of $\Omega$ and $\mathcal{T}$ is a touching function associated with $\mathcal{A}$. Let $(\mathcal{A}, \mathcal{T})$ be a partition of $\Omega$ with touching status. For $m \in \mathbb{N}$, a set $A$ of $\mathcal{A}$ is said to touch the boundary piece $\Gamma^{m}$ if and only if $\mathcal{T}(A, m)=1$. Thus an interface between $A$ and $\Gamma^{m}$ is taken into account only if $\mathcal{T}(A, m)=0$.

We denote by $\operatorname{TP}(\Omega)$ the set of all partitions of $\Omega$ with touching status whose perimeter is finite.

We next build a metric on $\operatorname{TP}(\Omega)$. We first define a (pseudo) metric $\operatorname{dist}_{L^{1}}$ on $\mathcal{B}(\Omega)$ by

$$
\forall A_{1}, A_{2} \in \mathcal{B}(\Omega) \quad \operatorname{dist}_{L^{1}}\left(A_{1}, A_{2}\right)=\mathcal{L}^{d}\left(A_{1} \Delta A_{2}\right) .
$$

Let $\mathcal{F}$ be the set of the functions from $\mathbb{N}$ to $\{0,1\}$ endowed with the product topology. This topology is metrizable, for instance it is compatible with the metric $\operatorname{dist}_{\mathcal{F}}$ defined by

$$
\forall T_{1}, T_{2} \in \mathcal{F} \quad \operatorname{dist}_{\mathcal{F}}\left(T_{1}, T_{2}\right)=\sum_{m \in \mathbb{N}} 2^{-m}\left|T_{1}(m)-T_{2}(m)\right|
$$


An arrangement of an element $(\mathcal{A}, \mathcal{T})$ of $\operatorname{TP}(\Omega)$ is a sequence $(A(i), T(i, \cdot), i \in \mathbb{N})$ of sets in $\mathcal{A} \cup\{\emptyset\}$ and functions in $\mathcal{F}$ such that:

- each set of $\mathcal{A}$ appears exactly once in the sequence $(A(i), i \in \mathbb{N})$ and the empty set $\emptyset$ appears countably many times in the sequence $(A(i), i \in \mathbb{N})$.

- for any $i \in \mathbb{N}$, if $A(i) \neq \emptyset$, then $T(i, \cdot)=\mathcal{T}(A(i), \cdot)$.

In particular, if $\mathcal{A}$ is finite, then $A(i)=\emptyset$ for $i$ sufficiently large. Whenever $A(i)=\emptyset$, the corresponding function $T(i, \cdot)$ might be an arbitrary element of $\mathcal{F}$. However we impose the global constraint on the functions $(T(i, \cdot), i \in \mathbb{N})$ that a boundary piece can touch at most one set, that is,

$$
\forall m \in \mathbb{N} \quad \sum_{i \in \mathbb{N}} T(i, m) \leqslant 1 .
$$

We define a metric Dist on $\operatorname{TP}(\Omega)$ by: for $\left(\mathcal{A}_{1}, \mathcal{T}_{1}\right),\left(\mathcal{A}_{2}, \mathcal{T}_{2}\right) \in \operatorname{TP}(\Omega)$

$$
\operatorname{Dist}\left(\left(\mathcal{A}_{1}, \mathcal{T}_{1}\right),\left(\mathcal{A}_{2}, \mathcal{T}_{2}\right)\right)=\inf \left\{\sum_{i \in \mathbb{N}}\left(\operatorname{dist}_{L^{1}}\left(A_{1}(i), A_{2}(i)\right)+\operatorname{dist}_{\mathcal{F}}\left(T_{1}(i, \cdot), T_{2}(i, \cdot)\right)\right)\right\},
$$

where the infimum is taken over all possible arrangements $\left(A_{j}(i), T_{j}(i, \cdot), i \in \mathbb{N}\right)$ of $\mathcal{A}_{j}$, $j=1,2$.

Our next aim is to define the surface energy corresponding to the surface tension $\tau$ (extracted from the microscopic model) for a partition with touching status. The results of this section are valid for any function $\tau$ from $S^{d-1}$ to $\mathbb{R}^{+}$satisfying the following hypothesis.

Hypothesis on $\tau$. - The function $\tau$ does not vanish on $S^{d-1}$ and it is invariant under sign change: $\forall x \in S^{d-1} 0<\tau(x)=\tau(-x)$. The homogeneous extension $\tau_{0}$ of $\tau$ to $\mathbb{R}^{d}$ defined by $\tau_{0}(0)=0$ and $\tau_{0}(x)=|x|_{2} \tau\left(x /|x|_{2}\right)$ for $x \in \mathbb{R}^{d} \backslash\{0\}$ is a convex function.

We define the surface energy of an element $(\mathcal{A}, \mathcal{T})$ of $\operatorname{TP}(\Omega)$ by

$$
\begin{aligned}
\mathcal{I}(\mathcal{A}, \mathcal{T})= & \sum_{A \in \mathcal{A}}\left(\frac{1}{2} \int_{\partial^{*} A \cap \Omega} \tau\left(v_{A}(x)\right) \mathrm{d} \mathcal{H}^{d-1}(x)\right. \\
& \left.+\sum_{m \in \mathbb{N}}(1-\mathcal{T}(A, m)) \int_{\partial^{*} A \cap \Gamma^{m}} \tau\left(v_{A}(x)\right) \mathrm{d} \mathcal{H}^{d-1}(x)\right) .
\end{aligned}
$$

The first term in the above formula corresponds to the interfaces present in $\Omega$, while the second term corresponds to the interfaces on $\Gamma$. Let $(\mathcal{A}, \mathcal{T}) \in \operatorname{TP}(\Omega)$. We define the reduced boundary $\partial^{*}(\mathcal{A}, \mathcal{T})$ of $(\mathcal{A}, \mathcal{T})$ to be the set

$\partial^{*}(\mathcal{A}, \mathcal{T})=\left(\bigcup_{A_{1}, A_{2} \in \mathcal{A}, A_{1} \neq A_{2}} \partial^{*} A_{1} \cap \partial^{*} A_{2}\right) \cup\left(\bigcup_{(A, m) \in \mathcal{A} \times \mathbb{N}: \mathcal{T}(A, m)=0} \partial^{*} A \cap \partial^{*} \Omega \cap \Gamma^{m}\right)$.

For a point $x$ of $\partial^{*}(\mathcal{A}, \mathcal{T}) \cap \Omega$, the pair $\left(A_{1}, A_{2}\right)$ of elements of $\mathcal{A}$ satisfying $A_{1} \neq A_{2}$, $x \in \partial^{*} A_{1} \cap \partial^{*} A_{2}$, is unique up to the order (both sets $A_{1}$ and $A_{2}$ have density $1 / 2$ at $x$ ); moreover, the generalized normal vectors of $A_{1}$ and $A_{2}$ at $x$ satisfy $v_{A_{1}}(x)+v_{A_{2}}(x)=0$. With each point $x$ of $\partial^{*}(\mathcal{A}, \mathcal{T}) \cap \Omega$, we associate the element $\bar{v}(x)$ of the projective sphere $P S^{d-1}$ corresponding to the vectors $v_{A_{1}}(x), v_{A_{2}}(x)$. We denote by $v(x)$ one among these two unit vectors, selected in such a way that the map $x \in \partial^{*}(\mathcal{A}, \mathcal{T}) \cap$ 
$\Omega \mapsto v(x) \in S^{d-1}$ is measurable with respect to $\left.\mathcal{H}^{d-1}\right|_{\partial^{*}(\mathcal{A}, \mathcal{T})}$ (a way to perform this construction is to choose an arrangement $(A(i), i \in \mathbb{N})$ of $\mathcal{A}$ and then to select at each point the normal unit vector corresponding to the set having the smallest index in the arrangement; more precisely, if $x$ belongs to $\partial^{*} A(i) \cap \partial^{*} A(j)$, where $i<j$, we set $\left.v(x)=v_{A(i)}(x)\right)$. In any case the map $x \in \partial^{*}(\mathcal{A}, \mathcal{T}) \cap \Omega \mapsto \bar{v}(x) \in P S^{d-1}$ is $\left.\mathcal{H}^{d-1}\right|_{\partial^{*}(\mathcal{A}, \mathcal{T})}$ measurable. Similarly, for a point $x$ of $\partial^{*}(\mathcal{A}, \mathcal{T}) \cap \Gamma$, there exist a unique set $A$ in $\mathcal{A}$ and a unique integer $m$ such that $\mathcal{T}(A, m)=0$ and $x \in \partial^{*} A \cap \Gamma^{m}$. Moreover, the generalized normal vectors of $A$ and $\Omega$ at $x$ satisfy $v_{A}(x)-v_{\Omega}(x)=0$. We define $v(x)=v_{A}(x)$, and $\bar{v}(x)$ is the projection of $v(x)$ on $P S^{d-1}$. Now the surface energy $\mathcal{I}(\mathcal{A}, \mathcal{T})$ can be rewritten as

$$
\mathcal{I}(\mathcal{A}, \mathcal{T})=\int_{\partial^{*}(\mathcal{A}, \mathcal{T}) \cap \Omega} \tau(\bar{v}(x)) \mathrm{d} \mathcal{H}^{d-1}(x)+\int_{\partial^{*}(\mathcal{A}, \mathcal{T}) \cap \Gamma} \tau(v(x)) \mathrm{d} \mathcal{H}^{d-1}(x)
$$

or even in the more concise form

$$
\mathcal{I}(\mathcal{A}, \mathcal{T})=\int_{\partial^{*}(\mathcal{A}, \mathcal{T})} \tau(\bar{v}(x)) \mathrm{d} \mathcal{H}^{d-1}(x) .
$$

The symmetry of the surface tension $\tau$ allows to define it on the projective sphere $P S^{d-1}$, so that $\tau(\bar{v}(x))$ makes sense. The agreement of the two expressions of the surface energy $\mathcal{I}(\mathcal{A}, \mathcal{T})$ is a consequence of the following fact: for any $A$ in $\mathcal{A}$,

$$
\mathcal{H}^{d-1}\left(\partial^{*} A\right)=\mathcal{H}^{d-1}\left(\partial^{*} A \cap \partial^{*} \Omega\right)+\sum_{E \in \mathcal{A} \backslash\{A\}} \mathcal{H}^{d-1}\left(\partial^{*} A \cap \partial^{*} E\right) .
$$

See [17], Lemma 1.4 and formula (1.5).

LEMMA 3.1. - Let $(\mathcal{A}, \mathcal{T})$ belong to $\operatorname{TP}(\Omega)$. Let $f: \partial^{*}(\mathcal{A}, \mathcal{T}) \mapsto \mathbb{R}$ be a $\left.\mathcal{H}^{d-1}\right|_{\partial^{*}(\mathcal{A}, \mathcal{T})}$ measurable bounded function. For $\mathcal{H}^{d-1}$ almost all $x$ in $\partial^{*}(\mathcal{A}, \mathcal{T})$,

$$
\lim _{r \rightarrow 0}\left(\alpha_{d-1} r^{d-1}\right)^{-1} \int_{B(x, r) \cap \partial^{*}(\mathcal{A}, \mathcal{T})} f(y) \mathrm{d} \mathcal{H}^{d-1}(y)=f(x) .
$$

Proof. - Since $\mathcal{A}$ is a partition of $\Omega$ having finite perimeter, then $\mathcal{H}^{d-1}\left(\partial^{*}(\mathcal{A}, \mathcal{T})\right)$ is finite, whence for $\mathcal{H}^{d-1}$ almost all $x$ in $\partial^{*}(\mathcal{A}, \mathcal{T})$ (by [25], Corollary 2.5),

$$
\limsup _{r \rightarrow 0}\left(\alpha_{d-1} r^{d-1}\right)^{-1} \mathcal{H}^{d-1}\left(B(x, r) \cap \partial^{*}(\mathcal{A}, \mathcal{T})\right) \leqslant 1 .
$$

We do the proof for the points in $\partial^{*}(\mathcal{A}, \mathcal{T}) \cap \Omega$, the argument is similar for the points in $\partial^{*}(\mathcal{A}, \mathcal{T}) \cap \Gamma$. Let $A$ be an element of $\mathcal{A}$. It follows from (20) that for $\mathcal{H}^{d-1}$ almost all $x$ in $\partial^{*} A \cap \partial^{*}(\mathcal{A}, \mathcal{T}) \cap \Omega$,

$$
\begin{aligned}
& \liminf _{r \rightarrow 0}\left(\alpha_{d-1} r^{d-1}\right)^{-1} \mathcal{H}^{d-1}\left(B(x, r) \cap \partial^{*}(\mathcal{A}, \mathcal{T})\right) \\
& \quad \geqslant \liminf _{r \rightarrow 0}\left(\alpha_{d-1} r^{d-1}\right)^{-1} \mathcal{H}^{d-1}\left(B(x, r) \cap \partial^{*} A\right)=1, \\
& \lim _{r \rightarrow 0}\left(\alpha_{d-1} r^{d-1}\right)^{-1} \mathcal{H}^{d-1}\left(B(x, r) \cap\left(\partial^{*} A \Delta \partial^{*}(\mathcal{A}, \mathcal{T})\right)\right)=0 .
\end{aligned}
$$


Since $\mathcal{A}$ is countable, then for $\mathcal{H}^{d-1}$ almost all $x$ in $\partial^{*}(\mathcal{A}, \mathcal{T}) \cap \Omega$,

$$
\begin{aligned}
& \lim _{r \rightarrow 0}\left(\alpha_{d-1} r^{d-1}\right)^{-1} \mathcal{H}^{d-1}\left(B(x, r) \cap \partial^{*}(\mathcal{A}, \mathcal{T})\right)=1, \\
& \lim _{r \rightarrow 0}\left(\alpha_{d-1} r^{d-1}\right)^{-1} \mathcal{H}^{d-1}\left(B(x, r) \cap\left(\partial^{*}(\mathcal{A}, \mathcal{T}) \Delta \partial^{*} A_{1}(x)\right)\right)=0,
\end{aligned}
$$

(where $A_{1}(x)$ is one of the 2 sets of $\mathcal{A}$ having density $1 / 2$ at $x$ ). Next, using the Besicovitch differentiation theorem (see the appendix), for $\mathcal{H}^{d-1}$ almost all $x$ in $\partial^{*}(\mathcal{A}, \mathcal{T}) \cap \Omega$,

$$
\lim _{r \rightarrow 0}\left(\alpha_{d-1} r^{d-1}\right)^{-1} \int_{B(x, r) \cap \partial^{*} A_{1}(x)} f(y) \mathrm{d} \mathcal{H}^{d-1}(y)=f(x) .
$$

By decomposing $B(x, r) \cap \partial^{*}(\mathcal{A}, \mathcal{T})$ as

$$
\begin{gathered}
\left(B(x, r) \cap \partial^{*} A_{1}(x)\right) \cup\left(B(x, r) \cap\left(\partial^{*}(\mathcal{A}, \mathcal{T}) \backslash \partial^{*} A_{1}(x)\right)\right) \\
\backslash\left(B(x, r) \cap\left(\partial^{*} A_{1}(x) \backslash \partial^{*}(\mathcal{A}, \mathcal{T})\right)\right)
\end{gathered}
$$

and integrating $f$ separately over each of these sets, with the help of the previous density results, we obtain the claim of the lemma for $\mathcal{H}^{d-1}$ almost all $x$ in $\partial^{*}(\mathcal{A}, \mathcal{T}) \cap \Omega$.

We check that

$$
\forall(\mathcal{A}, \mathcal{T}) \in \operatorname{TP}(\Omega) \quad \frac{1}{2} \tau_{\min }(\mathcal{P}(\mathcal{A})-\mathcal{P}(\Omega)) \leqslant \mathcal{I}(\mathcal{A}, \mathcal{T}) \leqslant \frac{1}{2} \tau_{\max }(\mathcal{P}(\mathcal{A})+\mathcal{P}(\Omega)),
$$

where $\tau_{\min }$ and $\tau_{\max }$ are the infimum and the supremum of $\tau$ on $S^{d-1}$. The hypothesis on $\tau$ implies that $0<\tau_{\min } \leqslant \tau_{\max }<\infty$. Therefore $\mathcal{I}(\mathcal{A}, \mathcal{T})$ is finite whenever $\mathcal{A}$ is a partition of $\Omega$ having finite perimeter.

LEMMA 3.2. - The surface energy $\mathcal{I}$ is lower semicontinuous with respect to the metric Dist.

Remark. - It seems that the general results of $[5,6]$ cannot be applied directly in this situation, because we are dealing with partitions having a countable number of sets. Therefore we provide a direct proof of the lower semicontinuity.

Proof. - Let $M \in \mathbb{N}$. For $m \in\{0, \ldots, M\}$, since $\Gamma^{m}$ is relatively open in $\Gamma$, there exists a set $V_{m}$, relatively open in $\Gamma$, such that

$$
\bar{V}_{m} \subset \Gamma^{m}, \quad \mathcal{H}^{d-1}\left(\Gamma^{m} \backslash V_{m}\right)<1 / M^{2} .
$$

As a consequence, there exists $\delta>0$ such that $\mathcal{V}\left(V_{m}, \delta\right) \cap \Gamma \subset \Gamma^{m}$ for each $m \in$ $\{0, \ldots, M\}$, and the sets $\overline{\mathcal{V}\left(V_{m}, \delta\right)}, m \in\{0, \ldots, M\}$, are pairwise disjoint. Let $m \in$ $\{0, \ldots, M\}$. Since $\mathcal{H}^{d-1}\left(V_{m}\right)$ is finite, by the definition of the Hausdorff measure $\mathcal{H}^{d-1}$, there exists a collection of balls $B\left(x_{i}, r_{i}\right), i \in I$, such that

$$
\begin{aligned}
\forall i \in I \quad & B\left(x_{i}, r_{i}\right) \cap V_{m} \neq \emptyset, \quad 0<r_{i}<\delta / 4 \\
& V_{m} \subset \bigcup_{i \in I} B\left(x_{i}, r_{i}\right), \quad \sum_{i \in I} r_{i}^{d-1}<\infty .
\end{aligned}
$$


Let $G_{m}=\bigcup_{i \in I} B\left(x_{i}, r_{i}\right) \backslash \Omega$. The sets $G_{m}, m \in\{0, \ldots, M\}$, are pairwise disjoint, moreover, $\left(\partial G_{m} \cup \mathcal{V}\left(V_{m}, \delta\right)\right) \cap \Gamma \subset \Gamma^{m}$ for each $m \in\{0, \ldots, M\}$. Let us define the map $\mathcal{I}_{M}: \operatorname{TP}(\Omega) \mapsto \mathbb{R}^{+}$by

$$
\begin{aligned}
\mathcal{I}_{M}(\mathcal{A}, \mathcal{T})= & \sum_{A \in \mathcal{A}}\left(\frac{1}{2} \int_{\partial^{*} A \cap \Omega} \tau\left(v_{A}(x)\right) \mathrm{d} \mathcal{H}^{d-1}(x)\right. \\
& \left.+\sum_{0 \leqslant m \leqslant M}(1-\mathcal{T}(A, m)) \int_{\partial^{*} A \cap G_{m}} \tau\left(v_{A}(x)\right) \mathrm{d} \mathcal{H}^{d-1}(x)\right) .
\end{aligned}
$$

We have then for all $M \in \mathbb{N}$

$$
\begin{aligned}
& \forall(\mathcal{A}, \mathcal{T}) \in \operatorname{TP}(\Omega) \\
& \quad 0 \leqslant \mathcal{I}(\mathcal{A}, \mathcal{T})-\mathcal{I}_{M}(\mathcal{A}, \mathcal{T}) \leqslant \tau_{\max } \mathcal{H}^{d-1}\left(\bigcup_{m>M} \Gamma^{m}\right)+\tau_{\max }(M+1) / M^{2},
\end{aligned}
$$

so that

$$
\forall(\mathcal{A}, \mathcal{T}) \in \operatorname{TP}(\Omega) \quad \mathcal{I}(\mathcal{A}, \mathcal{T})=\sup _{M \in \mathbb{N}} \mathcal{I}_{M}(\mathcal{A}, \mathcal{T})
$$

and we need only to prove that for a fixed $M \in \mathbb{N}$ the map $\mathcal{I}_{M}$ is lower semicontinuous. We denote by $C_{0}^{1}\left(\mathbb{R}^{d}, \mathcal{W}_{\tau}\right)$ the set of the compactly supported vector fields defined on $\mathbb{R}^{d}$ taking values in the Wulff crystal $\mathcal{W}_{\tau}$. Let $(\mathcal{A}, \mathcal{T}) \in \operatorname{TP}(\Omega)$. We claim that, up to an additive constant, $2 \mathcal{I}_{M}(\mathcal{A}, \mathcal{T})$ is equal to the supremum of the quantity

$$
\begin{gathered}
\sum_{A \in \mathcal{A}} \int\left(\chi_{A}(x)+\sum_{0 \leqslant m \leqslant M} \mathcal{T}(A, m) \chi_{G_{m}}(x)\right) \operatorname{div} f_{A}(x) \mathrm{d} x \\
+\sum_{0 \leqslant m \leqslant M}\left(1-\sum_{A \in \mathcal{A}} \mathcal{T}(A, m)\right) \int \chi_{G_{m}}(x) \operatorname{div} f(x) \mathrm{d} x
\end{gathered}
$$

over all families of vector fields $f, f_{A}, A \in \mathcal{A}$, belonging to $C_{0}^{1}\left(\mathbb{R}^{d}, \mathcal{W}_{\tau}\right)$. Indeed, the surface energy $\mathcal{I}(E)$ of a set $E$ of finite perimeter is (see [15])

$$
\mathcal{I}(E)=\sup \left\{\int_{E} \operatorname{div} f(x) \mathrm{d} x: f \in C_{0}^{1}\left(\mathbb{R}^{d}, \mathcal{W}_{\tau}\right)\right\}=\int_{\partial^{*} E} \tau\left(v_{E}(x)\right) \mathrm{d} \mathcal{H}^{d-1}(x) .
$$

Thus the supremum of the previous quantity is equal to

$$
\left[\sum_{A \in \mathcal{A}} \mathcal{I}\left(A \cup \underset{\substack{m \in\{0, \ldots M\} \\ \mathcal{T}(A, m)=1}}{\bigcup} G_{m}\right)\right]+\mathcal{I}\left(\bigcup_{\sum_{A \in \mathcal{A}} \mathcal{T}(A, m)=0} G_{m}\right)
$$

which is further equal to $2 \mathcal{I}_{M}(\mathcal{A}, \mathcal{T})+c$, where $c$ is the constant

$$
c=\sum_{0 \leqslant m \leqslant M} \int_{\partial^{*} G_{m} \backslash \Gamma} \tau\left(v_{G_{m}}(x)\right) \mathrm{d} \mathcal{H}^{d-1}(x)+\int_{\Gamma \backslash \bigcup_{0 \leqslant m \leqslant M} G_{m}} \tau\left(v_{\Omega}(x)\right) \mathrm{d} \mathcal{H}^{d-1}(x) .
$$

We finally prove the lower semicontinuity of $\mathcal{I}_{M}$ with the help of the previous representation. Let $(\mathcal{A}, \mathcal{T}) \in \operatorname{TP}(\Omega)$ and let $(A(i), T(i, \cdot))_{i \in \mathbb{N}}$ be an arrangement of 
$(\mathcal{A}, \mathcal{T})$ such that $T(i, \cdot)$ is the null function whenever $A(i)=\emptyset$. Let $\varepsilon>0$. There exists a finite subset $I$ of $\mathbb{N}$ and a finite family of vector fields $f,\left(f_{i}\right)_{i \in I}$ in $C_{0}^{1}\left(\mathbb{R}^{d}, \mathcal{W}_{\tau}\right)$ such that

$$
\begin{gathered}
\forall m \in\{0, \ldots, M\} \quad \sum_{i \in I} T(i, m)=\sum_{i \in \mathbb{N}} T(i, m), \\
2 \mathcal{I}_{M}(\mathcal{A}, \mathcal{T})+c-\varepsilon \leqslant \sum_{i \in I} \int\left(\chi_{A(i)}(x)+\sum_{0 \leqslant m \leqslant M} T(i, m) \chi_{G_{m}}(x)\right) \operatorname{div} f_{i}(x) \mathrm{d} x \\
+\sum_{0 \leqslant m \leqslant M}\left(1-\sum_{i \in \mathbb{N}} T(i, m)\right) \int \chi_{G_{m}}(x) \operatorname{div} f(x) \mathrm{d} x .
\end{gathered}
$$

Let

$$
a=\max \left(\sup _{i \in I} \sup _{x \in \mathbb{R}^{d}}\left|\operatorname{div} f_{i}(x)\right|, \sup _{x \in \mathbb{R}^{d}}|\operatorname{div} f(x)|\right) .
$$

Let $\delta$ be such that $0<\delta<\min \left(2^{-M}, \varepsilon / a\right)$ and let $\left(\mathcal{A}^{\prime}, \mathcal{T}^{\prime}\right)$ be an element of $\operatorname{TP}(\Omega)$ such that

$$
\operatorname{Dist}\left((\mathcal{A}, \mathcal{T}),\left(\mathcal{A}^{\prime}, \mathcal{T}^{\prime}\right)\right)<\delta
$$

Then there exists an arrangement $\left(A^{\prime}(i), T^{\prime}(i, \cdot)\right)_{i \in \mathbb{N}}$ of $\left(\mathcal{A}^{\prime}, \mathcal{T}^{\prime}\right)$ such that

$$
\sum_{i \in \mathbb{N}}\left(\operatorname{dist}_{L^{1}}\left(A(i), A^{\prime}(i)\right)+\operatorname{dist}_{\mathcal{F}}\left(T(i, \cdot), T^{\prime}(i, \cdot)\right)\right)<\delta .
$$

Then we must have

$$
\forall i \in \mathbb{N} \quad \forall m \in\{0, \ldots, M\} \quad T(i, m)=T^{\prime}(i, m),
$$

and thus

$$
\begin{aligned}
& 2 \mathcal{I}_{M}(\mathcal{A}, \mathcal{T})+c-\varepsilon \\
& \leqslant \sum_{i \in I} \int\left(\chi_{A^{\prime}(i)}(x)+\sum_{0 \leqslant m \leqslant M} T^{\prime}(i, m) \chi_{G_{m}}(x)\right) \operatorname{div} f_{i}(x) \mathrm{d} x \\
& \quad+\sum_{0 \leqslant m \leqslant M} \int\left(1-\sum_{i \in \mathbb{N}} T^{\prime}(i, m)\right) \chi_{G_{m}}(x) \operatorname{div} f(x) \mathrm{d} x+a \sum_{i \in I} \mathcal{L}^{d}\left(A^{\prime}(i) \Delta A(i)\right) \\
& \leqslant 2 \mathcal{I}_{M}\left(\mathcal{A}^{\prime}, \mathcal{T}^{\prime}\right)+c+a \delta \leqslant 2 \mathcal{I}_{M}\left(\mathcal{A}^{\prime}, \mathcal{T}^{\prime}\right)+c+\varepsilon
\end{aligned}
$$

which proves the lower semicontinuity of $\mathcal{I}_{M}$ at $(\mathcal{A}, \mathcal{T})$.

Proposition 3.3. - The map $(\mathcal{A}, \mathcal{T}) \in(\operatorname{TP}(\Omega)$, Dist $) \mapsto \mathcal{I}(\mathcal{A}, \mathcal{T}) \in \mathbb{R}^{+}$is a good rate function, i.e., its level sets $\{(\mathcal{A}, \mathcal{T}) \in \operatorname{TP}(\Omega): \mathcal{I}(\mathcal{A}, \mathcal{T}) \leqslant \lambda\}, \lambda \in \mathbb{R}^{+}$, are compact with respect to the metric Dist.

Proof. - The proof is a variant of the proof of the compactness result for Caccioppoli partitions of Congedo and Tamanini [17], Theorem 1.6. The only additional problem is the touching function. Let $\lambda$ belong to $\mathbb{R}^{+}$and let $\left(\mathcal{A}_{n}, \mathcal{T}_{n}\right)_{n \in \mathbb{N}}$ be a sequence in $\operatorname{TP}(\Omega)$ such that $\mathcal{I}\left(\mathcal{A}_{n}, \mathcal{T}_{n}\right) \leqslant \lambda$ for all $n$ in $\mathbb{N}$. For any $n \in \mathbb{N}$, since 


$$
\begin{aligned}
& \sum_{A \in \mathcal{A}} \mathcal{L}^{d}(A) \leqslant \mathcal{L}^{d}(\Omega) \\
& \sum_{A \in \mathcal{A}} \mathcal{P}(A) \leqslant\left(2 / \tau_{\min }\right) \mathcal{I}(\mathcal{A}, \mathcal{T})+\mathcal{P}(\Omega) \leqslant 2 \lambda / \tau_{\min }+\mathcal{P}(\Omega) \\
& \sum_{A \in \mathcal{A}} \sum_{m \in \mathbb{N}} 2^{-m} \mathcal{T}(A, m) \leqslant 2
\end{aligned}
$$

then, for $t$ positive, there is a finite number of sets $A$ in $\mathcal{A}_{n}$ such that

$$
\mathcal{L}^{d}(A)+\mathcal{P}(A)+\sum_{m \in \mathbb{N}} 2^{-m} \mathcal{T}(A, m)>t .
$$

Therefore there exists an arrangement $\left(A_{n}(i), T_{n}(i, \cdot), i \in \mathbb{N}\right)$ of $\mathcal{A}_{n}$ such that $A_{n}(2 i+$ $1)=\emptyset, T_{n}(2 i+1, \cdot)=0_{\mathcal{F}}$ for $i \in \mathbb{N}\left(0_{\mathcal{F}}\right.$ is the null function of $\left.\mathcal{F}\right)$ and if we set

$$
\forall i \in \mathbb{N} \quad u_{n}(i)=\mathcal{L}^{d}\left(A_{n}(2 i)\right)+\mathcal{P}\left(A_{n}(2 i)\right)+\sum_{m \in \mathbb{N}} 2^{-m} T_{n}(2 i, m),
$$

then the sequence $\left(u_{n}(i)\right)_{i \in \mathbb{N}}$ is decreasing. Yet the space $\{E \in \mathcal{B}(\Omega): \mathcal{P}(E) \leqslant \lambda\}$ is compact with respect to the metric $\operatorname{dist}_{L^{1}}$ (see the appendix). The space $\left(\mathcal{F}, \operatorname{dist}_{\mathcal{F}}\right)$ is also compact. By a standard diagonal argument, we can extract from the sequence of arrangements $\left(A_{n}(i), T_{n}(i, \cdot), i \in \mathbb{N}\right)_{n \in \mathbb{N}}$ a subsequence (not relabeled) such that: for each $i$ in $\mathbb{N}$, there exist a Borel set $A(2 i)$ in $\mathcal{B}(\Omega)$ and a map $T_{i}$ in $\mathcal{F}$ such that

$$
\lim _{n \rightarrow \infty} \operatorname{dist}_{L^{1}}\left(A_{n}(2 i), A(2 i)\right)+\operatorname{dist}_{\mathcal{F}}\left(T_{n}(2 i, \cdot), T_{i}\right)=0 .
$$

For $i$ odd we set $A(i)=\emptyset$. For any $i_{1} \neq i_{2}$ and $n$ in $\mathbb{N}$,

$$
\mathcal{L}^{d}\left(A\left(i_{1}\right) \cap A\left(i_{2}\right)\right) \leqslant \operatorname{dist}_{L^{1}}\left(A\left(i_{1}\right), A_{n}\left(i_{1}\right)\right)+\operatorname{dist}_{L^{1}}\left(A_{n}\left(i_{2}\right), A\left(i_{2}\right)\right) .
$$

Letting $n$ go to $\infty$ we obtain that $A\left(i_{1}\right) \cap A\left(i_{2}\right)$ is negligible for any $i_{1} \neq i_{2}$. Let $\mathcal{A}$ be the collection of the nonnegligible sets of the sequence $(A(i), i \in \mathbb{N})$, that is, $\mathcal{A}=\{A(i): i \in \mathbb{N}\} \backslash\{\emptyset\}$. Then $(A(i), i \in \mathbb{N})$ is an arrangement of $\mathcal{A}$. Next, we have

$$
\forall n, i \in \mathbb{N} \quad \mathcal{L}^{d}(\Omega)+\mathcal{P}(\Omega)+2 \lambda / \tau_{\min }+2 \geqslant i u_{n}(i) .
$$

We set $a=\mathcal{L}^{d}(\Omega)+\mathcal{P}(\Omega)+2 \lambda / \tau_{\min }+2$. By the isoperimetric inequality in $\mathbb{R}^{d}$, for all $n, i$ in $\mathbb{N}$, we have

$$
\mathcal{L}^{d}\left(A_{n}(2 i)\right) \leqslant c_{\text {iso }} \mathcal{P}\left(A_{n}(2 i)\right)^{d /(d-1)} \leqslant c_{\text {iso }}(a / i)^{d /(d-1)} .
$$

By summing the isoperimetric inequality, we get

$$
\forall n \in \mathbb{N} \quad \mathcal{L}^{d}(\Omega)=\sum_{A \in \mathcal{A}_{n}} \mathcal{L}^{d}(A) \leqslant \sum_{0 \leqslant k \leqslant 2 i} \mathcal{L}^{d}\left(A_{n}(k)\right)+(d-1) c_{\text {iso }} a^{d /(d-1)} i^{-1 /(d-1)} .
$$

By letting successively $n$ and $i$ go to infinity, we get $\mathcal{L}^{d}(\Omega) \leqslant \sum_{A \in \mathcal{A}} \mathcal{L}^{d}(A)$. By the lower semicontinuity of the perimeter and Fatou's Lemma,

$$
\begin{aligned}
\mathcal{P}(\mathcal{A}) & =\sum_{i \in \mathbb{N}} \mathcal{P}(A(2 i)) \leqslant \sum_{i \in \mathbb{N}} \liminf _{n \rightarrow \infty} \mathcal{P}\left(A_{n}(2 i)\right) \\
& \leqslant \liminf _{n \rightarrow \infty} \sum_{i \in \mathbb{N}} \mathcal{P}\left(A_{n}(2 i)\right)=\liminf _{n \rightarrow \infty} \mathcal{P}\left(\mathcal{A}_{n}\right) \leqslant 2 \lambda / \tau_{\min }+\mathcal{P}(\Omega)<\infty .
\end{aligned}
$$


Thus $\mathcal{A}$ is a partition of $\Omega$ having finite perimeter. Let $A$ belong to $\mathcal{A}$. There exists a unique index $i$ such that $A=A(2 i)$. We set $\mathcal{T}(A, \cdot)=T_{i}$. Let us check that $\mathcal{T}$ satisfies the compatibility condition. For any $m \in \mathbb{N}$, by Fatou Lemma,

$$
\begin{aligned}
\sum_{A \in \mathcal{A}} \mathcal{T}(A, m) & =\sum_{i \in \mathbb{N}} T_{i}(m)=\sum_{i \in \mathbb{N}} \lim _{n \rightarrow \infty} \mathcal{T}_{n}\left(A_{n}(i), m\right) \\
& \leqslant \liminf _{n \rightarrow \infty} \sum_{i \in \mathbb{N}} \mathcal{T}_{n}\left(A_{n}(i), m\right)=\liminf _{n \rightarrow \infty} \sum_{A \in \mathcal{A}_{n}} \mathcal{T}_{n}(A, m) \leqslant 1 .
\end{aligned}
$$

Thus $\mathcal{T}$ is a touching function for $\mathcal{A}$. It remains to check that the subsequence $\left(\mathcal{A}_{n}, \mathcal{T}_{n}\right)_{n \in \mathbb{N}}$ converges towards $(\mathcal{A}, \mathcal{T})$ with respect to Dist. Setting

$$
\forall i \in \mathbb{N} \quad A(2 i+1)=\emptyset, \quad T(2 i+1, \cdot)=0_{\mathcal{F}}, \quad T(2 i, \cdot)=T_{i}
$$

we see that $(A(i), T(i, \cdot), i \in \mathbb{N})$ is an arrangement of $(\mathcal{A}, \mathcal{T})$. For all $n, i \in \mathbb{N}$,

$$
\sum_{m \in \mathbb{N}} 2^{-m} T_{n}(2 i, m) \leqslant a / i \text {. }
$$

Let $i$ be strictly larger than $a$ and let $\phi(i)$ be the unique integer such that $2^{-\phi(i)}>a / i \geqslant$ $2^{-\phi(i)-1}$. Then, for any $n \in \mathbb{N}$ and any $m \leqslant \phi(i)$, we have $T_{n}(2 i, m)=0$ so that

$$
\begin{aligned}
\sum_{k>2 i} \sum_{m \in \mathbb{N}} 2^{-m} T_{n}(2 k, m) & =\sum_{k>2 i} \sum_{m>\phi(i)} 2^{-m} T_{n}(2 k, m) \\
& =\sum_{m>\phi(i)} 2^{-m} \sum_{k>2 i} T_{n}(2 k, m) \leqslant \sum_{m>\phi(i)} 2^{-m} \leqslant 2^{-\phi(i)} .
\end{aligned}
$$

Therefore, for any $n, i$ in $\mathbb{N}$

$$
\begin{aligned}
\operatorname{Dist}\left(\left(\mathcal{A}_{n}, \mathcal{T}_{n}\right),(\mathcal{A}, \mathcal{T})\right) \leqslant & \sum_{0 \leqslant k \leqslant 2 i}\left(\operatorname{dist}_{L^{1}}\left(A_{n}(k), A(k)\right)+\operatorname{dist}_{\mathcal{F}}\left(T_{n}(k, \cdot), T(k, \cdot)\right)\right) \\
& +2(d-1) c_{\text {iso }} a^{d /(d-1)} i^{-1 /(d-1)}+2^{1-\phi(i)} .
\end{aligned}
$$

Letting first $n$ and then $i$ go to $\infty$ in this inequality, we see that the subsequence $\left(\mathcal{A}_{n}, \mathcal{T}_{n}\right)_{n \in \mathbb{N}}$ converges towards $(\mathcal{A}, \mathcal{T})$ in $(\operatorname{TP}(\Omega)$, Dist).

LEMMA 3.4. - Let $(\mathcal{A}, \mathcal{T})$ belong to $\operatorname{TP}(\Omega)$. For any positive $\varepsilon, \delta$, there exists a finite collection of disjoint balls $B\left(x_{i}, r_{i}\right), i \in I_{0} \cup I_{1}$, such that:

- for any $i \in I_{0}, B\left(x_{i}, r_{i}\right) \subseteq \Omega, 0<r_{i} \leqslant 1$, and there exist $A_{-}^{i}, A_{+}^{i} \in \mathcal{A}, v_{i} \in S^{d-1}$ such that

$$
\begin{aligned}
& \operatorname{dist}_{L^{1}}\left(A_{-}^{i} \cap B\left(x_{i}, r_{i}\right), B_{-}\left(x_{i}, r_{i}, v_{i}\right)\right) \leqslant \delta r_{i}^{d}, \\
& \operatorname{dist}_{L^{1}}\left(A_{+}^{i} \cap B\left(x_{i}, r_{i}\right), B_{+}\left(x_{i}, r_{i}, v_{i}\right)\right) \leqslant \delta r_{i}^{d},
\end{aligned}
$$

- for any $i \in I_{1}, x_{i} \in \Gamma, 0<r_{i} \leqslant 1$, and there exist $A_{i} \in \mathcal{A}, v_{i} \in S^{d-1}, m_{i} \in \mathbb{N}$, such that

$$
\begin{aligned}
& \mathcal{T}\left(A_{i}, m_{i}\right)=0, \quad B\left(x_{i}, r_{i}\right) \cap \Gamma \subset \Gamma^{m_{i}}, \\
& \operatorname{dist}_{L^{1}}\left(A_{i} \cap B\left(x_{i}, r_{i}\right), B_{-}\left(x_{i}, r_{i}, v_{i}\right)\right) \leqslant \delta r_{i}^{d}, \\
& \operatorname{dist}_{L^{1}}\left(B\left(x_{i}, r_{i}\right) \backslash \Omega, B_{+}\left(x_{i}, r_{i}, v_{i}\right)\right) \leqslant \delta r_{i}^{d} .
\end{aligned}
$$


Finally we have

$$
\left|\mathcal{I}(\mathcal{A}, \mathcal{T})-\sum_{i \in I_{0} \cup I_{1}} \alpha_{d-1} r_{i}^{d-1} \tau\left(v_{i}\right)\right| \leqslant \varepsilon .
$$

Proof. - Let $\varepsilon, \delta$ be positive with $\varepsilon<1 / 2, \delta<1$. Because a generalized normal vector is also a measure theoretic normal (see the appendix for the definition), for any $x$ in $\partial^{*}(\mathcal{A}, \mathcal{T}) \cap \Omega$, there exist a positive $r_{0}(x, \delta)$ and two sets $A_{-}^{x}, A_{+}^{x}$ in $\mathcal{A}$ such that, for any $r<r_{0}(x, \delta)$,

$$
\begin{aligned}
& \operatorname{dist}_{L^{1}}\left(A_{-}^{x} \cap B(x, r), B_{-}(x, r, v(x))\right) \leqslant \delta r^{d}, \\
& \operatorname{dist}_{L^{1}}\left(A_{+}^{x} \cap B(x, r), B_{+}(x, r, v(x))\right) \leqslant \delta r^{d} .
\end{aligned}
$$

We handle $\partial^{*}(\mathcal{A}, \mathcal{T}) \cap \Gamma$ in a similar fashion. For any $x$ in $\partial^{*}(\mathcal{A}, \mathcal{T}) \cap \Gamma$, there exist one set $A^{x}$ in $\mathcal{A}$, an integer $m$ in $\mathbb{N}$ such that $\mathcal{T}\left(A^{x}, m\right)=0$ and a positive $r_{1}(x, \delta)$ such that for any $r<r_{1}(x, \delta)$,

$$
\begin{aligned}
& B(x, r) \cap \Gamma \subseteq \Gamma^{m}, \\
& \operatorname{dist}_{L^{1}}\left(A^{x} \cap B(x, r), B_{-}(x, r, v(x))\right) \leqslant \delta r^{d}, \\
& \operatorname{dist}_{L^{1}}\left(B(x, r) \backslash \Omega, B_{+}(x, r, v(x))\right) \leqslant \delta r^{d} .
\end{aligned}
$$

The map $x \in \partial^{*}(\mathcal{A}, \mathcal{T}) \mapsto \bar{v}(x) \in P S^{d-1}$ is measurable with respect to $\left.\mathcal{H}^{d-1}\right|_{\partial^{*}(\mathcal{A}, \mathcal{T})}$. By Lemma 3.1, for $\mathcal{H}^{d-1}$ almost all $x$ in $\partial^{*}(\mathcal{A}, \mathcal{T})$,

$$
\begin{aligned}
& \lim _{r \rightarrow 0}\left(\alpha_{d-1} r^{d-1}\right)^{-1} \mathcal{H}^{d-1}\left(B(x, r) \cap \partial^{*}(\mathcal{A}, \mathcal{T})\right)=1, \\
& \lim _{r \rightarrow 0}\left(\alpha_{d-1} r^{d-1}\right)^{-1} \int_{B(x, r) \cap \partial^{*}(\mathcal{A}, \mathcal{T})} \tau(\bar{v}(y)) \mathrm{d} \mathcal{H}^{d-1}(y)=\tau(\bar{v}(x)) .
\end{aligned}
$$

Let $\partial^{* *}(\mathcal{A}, \mathcal{T})$ be the set of the points of $\partial^{*}(\mathcal{A}, \mathcal{T})$ where the two preceding identities hold simultaneously. Clearly $\mathcal{H}^{d-1}\left(\partial^{*}(\mathcal{A}, \mathcal{T}) \backslash \partial^{* *}(\mathcal{A}, \mathcal{T})\right)=0$. For any $x$ in $\partial^{* *}(\mathcal{A}, \mathcal{T})$, there exists a positive $r_{2}(x, \varepsilon)$ such that, for any $r<r_{2}(x, \varepsilon)$,

$$
\begin{aligned}
& \left|\mathcal{H}^{d-1}\left(B(x, r) \cap \partial^{*}(\mathcal{A}, \mathcal{T})\right)-\alpha_{d-1} r^{d-1}\right| \leqslant \varepsilon \alpha_{d-1} r^{d-1}, \\
& \left|\left(\alpha_{d-1} r^{d-1}\right)^{-1} \int_{B(x, r) \cap \partial^{*}(\mathcal{A}, \mathcal{T})} \tau(\bar{\nu}(y)) \mathrm{d} \mathcal{H}^{d-1}(y)-\tau(\bar{v}(x))\right| \leqslant \varepsilon .
\end{aligned}
$$

The family of balls

$$
\begin{array}{ll}
B(x, r), & x \in \partial^{* *}(\mathcal{A}, \mathcal{T}) \cap \Omega, \quad r<\min \left(r_{0}(x, \delta), r_{2}(x, \varepsilon), 1, d_{2}(x, \Gamma)\right), \\
B(x, r), & x \in \partial^{* *}(\mathcal{A}, \mathcal{T}) \cap \Gamma, \quad r<\min \left(r_{1}(x, \delta), r_{2}(x, \varepsilon), 1\right),
\end{array}
$$

is a Vitali relation for $\partial^{* *}(\mathcal{A}, \mathcal{T})$. By the standard Vitali covering theorem (see Theorem A.2), we may select a finite or countable collection of disjoint balls $B\left(x_{i}, r_{i}\right)$, $i \in I$, such that: for any $i$ in $I$,

- either $x_{i}$ belongs to $\partial^{* *}(\mathcal{A}, \mathcal{T}) \cap \Omega$ and $r_{i}<\min \left(r_{0}\left(x_{i}, \delta\right), r_{2}\left(x_{i}, \varepsilon\right), 1, d_{2}\left(x_{i}, \Gamma\right)\right)$,

- or $x_{i}$ belongs to $\partial^{* *}(\mathcal{A}, \mathcal{T}) \cap \Gamma$ and $r_{i}<\min \left(r_{1}\left(x_{i}, \delta\right), r_{2}\left(x_{i}, \varepsilon\right), 1\right)$, 
and moreover

$$
\text { either } \quad \mathcal{H}^{d-1}\left(\partial^{* *}(\mathcal{A}, \mathcal{T}) \backslash \bigcup_{i \in I} B\left(x_{i}, r_{i}\right)\right)=0 \quad \text { or } \quad \sum_{i \in I} r_{i}^{d-1}=\infty
$$

Because for each $i$ in $I, r_{i}$ is smaller than $r_{2}\left(x_{i}, \varepsilon\right)$,

$$
(1-\varepsilon) \sum_{i \in I} \alpha_{d-1} r_{i}^{d-1} \leqslant \mathcal{H}^{d-1}\left(\partial^{* *}(\mathcal{A}, \mathcal{T})\right) \leqslant \mathcal{P}(\mathcal{A})<\infty,
$$

and therefore the first case occurs, so that we may select two disjoint finite subsets $I_{0}, I_{1}$ of $I$ such that

$$
\begin{aligned}
& x_{i} \in \partial^{* *}(\mathcal{A}, \mathcal{T}) \cap \Omega \quad \text { if } i \in I_{0}, \quad x_{i} \in \partial^{* *}(\mathcal{A}, \mathcal{T}) \cap \Gamma \quad \text { if } i \in I_{1}, \\
& \mathcal{H}^{d-1}\left(\partial^{* *}(\mathcal{A}, \mathcal{T}) \backslash \bigcup_{i \in I_{0} \cup I_{1}} B\left(x_{i}, r_{i}\right)\right)<\varepsilon \mathcal{H}^{d-1}\left(\partial^{* *}(\mathcal{A}, \mathcal{T})\right) .
\end{aligned}
$$

We claim that the collection of balls $B\left(x_{i}, r_{i}\right), i \in I_{0} \cup I_{1}$, enjoys the desired properties. In fact, we need only to check the final inequality stated in the lemma. We compute

$$
\begin{aligned}
& \left|\int_{\partial^{*}(\mathcal{A}, \mathcal{T})} \tau(\bar{v}(x)) \mathrm{d} \mathcal{H}^{d-1}(x)-\sum_{i \in I_{0} \cup I_{1}} \alpha_{d-1} r_{i}^{d-1} \tau\left(\bar{v}\left(x_{i}\right)\right)\right| \\
& \leqslant \int_{\partial^{* *}(\mathcal{A}, \mathcal{T}) \backslash \bigcup_{i \in I_{0} \cup I_{1}} B\left(x_{i}, r_{i}\right)} \tau(\overline{\mathcal{v}}(x)) \mathrm{d} \mathcal{H}^{d-1}(x) \\
& \quad+\left.\sum_{i \in I_{0} \cup I_{1}}\right|_{\partial^{* *}(\mathcal{A}, \mathcal{T}) \cap B\left(x_{i}, r_{i}\right)} \tau(\overline{\mathcal{v}}(x)) \mathrm{d} \mathcal{H}^{d-1}(x)-\alpha_{d-1} r_{i}^{d-1} \tau\left(\overline{\mathcal{v}}\left(x_{i}\right)\right) \mid .
\end{aligned}
$$

The first integral of the right-hand member is less than $\varepsilon \mathcal{H}^{d-1}\left(\partial^{* *}(\mathcal{A}, \mathcal{T})\right) \tau_{\max }$. For any $i$ in $I_{0} \cup I_{1}$,

$$
\begin{aligned}
& \left|\int_{\partial^{* *}(\mathcal{A}, \mathcal{T}) \cap B\left(x_{i}, r_{i}\right)} \tau(\bar{v}(x)) \mathrm{d} \mathcal{H}^{d-1}(x)-\alpha_{d-1} r_{i}^{d-1} \tau\left(\bar{v}\left(x_{i}\right)\right)\right| \\
& \leqslant 2 \varepsilon \mathcal{H}^{d-1}\left(B\left(x_{i}, r_{i}\right) \cap \partial^{* *}(\mathcal{A}, \mathcal{T})\right),
\end{aligned}
$$

whence by summing over $i$ in $I_{0} \cup I_{1}$,

$$
\left.\sum_{i \in I_{0} \cup I_{1}}\right|_{\partial^{* *}(\mathcal{A}, \mathcal{T}) \cap B\left(x_{i}, r_{i}\right)} \tau(\bar{v}(x)) \mathrm{d} \mathcal{H}^{d-1}(x)-\alpha_{d-1} r_{i}^{d-1} \tau\left(\bar{v}\left(x_{i}\right)\right) \mid \leqslant 2 \varepsilon \mathcal{H}^{d-1}\left(\partial^{* *}(\mathcal{A}, \mathcal{T})\right)
$$

and putting these inequalities together, we get

$\left|\int_{\partial *(\mathcal{A}, \mathcal{T})} \tau(\overline{\mathcal{V}}(x)) \mathrm{d} \mathcal{H}^{d-1}(x)-\sum_{i \in I_{0} \cup I_{1}} \alpha_{d-1} r_{i}^{d-1} \tau\left(\overline{\mathcal{v}}\left(x_{i}\right)\right)\right| \leqslant \varepsilon \mathcal{H}^{d-1}\left(\partial^{* *}(\mathcal{A}, \mathcal{T})\right)\left(\tau_{\max }+2\right)$.

Since $\mathcal{H}^{d-1}\left(\partial^{* *}(\mathcal{A}, \mathcal{T})\right) \leqslant \mathcal{I}(\mathcal{A}, \mathcal{T}) / \tau_{\text {min }}$, we get

$$
\left|\mathcal{I}(\mathcal{A}, \mathcal{T})-\sum_{i \in I_{0} \cup I_{1}} \alpha_{d-1} r_{i}^{d-1} \tau\left(\bar{v}\left(x_{i}\right)\right)\right| \leqslant \varepsilon\left(2+\tau_{\max }\right) \mathcal{I}(\mathcal{A}, \mathcal{T}) / \tau_{\min } .
$$


Since $\tau_{\max }, \tau_{\min }$ and $\mathcal{I}(\mathcal{A}, \mathcal{T})$ are fixed and finite, we have the required estimate.

An element $(\mathcal{A}, \mathcal{T})$ of $\operatorname{TP}(\Omega)$ is said to be polyhedral if $\mathcal{A}$ contains a finite number of sets and $\partial^{*}(\mathcal{A}, \mathcal{T}) \cap \Omega$ is included in the union of a finite number of hyperplanes. An hypersurface is a $C^{1}$ submanifold of $\mathbb{R}^{d}$ of codimension 1 .

THEOREM 3.5. - Let $(\mathcal{A}, \mathcal{T})$ belong to $\operatorname{TP}(\Omega)$. For any $\varepsilon>0$, there exists a polyhedral element $\left(\mathcal{A}^{\prime}, \mathcal{T}^{\prime}\right)$ in $\operatorname{TP}(\Omega)$ such that

$\operatorname{Dist}\left((\mathcal{A}, \mathcal{T}),\left(\mathcal{A}^{\prime}, \mathcal{T}^{\prime}\right)\right)<\varepsilon, \quad\left|\mathcal{I}(\mathcal{A}, \mathcal{T})-\mathcal{I}\left(\mathcal{A}^{\prime}, \mathcal{T}^{\prime}\right)\right|<\varepsilon, \quad \mathcal{H}^{d-1}\left(\overline{\partial^{*}\left(\mathcal{A}^{\prime}, \mathcal{T}^{\prime}\right)} \cap \Gamma\right)<\varepsilon$.

Proof. - We first reduce the problem to a partition having a finite number of elements. Let $(\mathcal{A}, \mathcal{T})$ belong to $\operatorname{TP}(\Omega)$ and let $\varepsilon>0$. There exists a finite number of sets $A_{1}, \ldots, A_{r}$ in $\mathcal{A}$ such that

$$
\sum_{A \in \mathcal{A} \backslash\left\{A_{1}, \ldots, A_{r}\right\}} \mathcal{L}^{d}(A)+\mathcal{H}^{d-1}\left(\partial^{*} A \cap \Gamma\right)<\varepsilon .
$$

Let $\left(\mathcal{A}^{\prime}, \mathcal{T}^{\prime}\right)$ be the element of $\operatorname{TP}(\Omega)$ defined by:

$$
\begin{gathered}
\mathcal{A}^{\prime}=\left\{A_{1}, \ldots, A_{r}, \Omega \backslash \bigcup_{1 \leqslant i \leqslant r} A_{i}\right\}, \\
\forall i \in\{1, \ldots, r\} \quad \forall m \in \mathbb{N} \quad \mathcal{T}^{\prime}\left(A_{i}, m\right)=\mathcal{T}\left(A_{i}, m\right), \\
\forall m \in \mathbb{N} \quad \mathcal{T}^{\prime}\left(\Omega \backslash \bigcup_{1 \leqslant i \leqslant r} A_{i}, m\right)=\mathcal{T}\left(A_{0}, m\right),
\end{gathered}
$$

where $A_{0}$ is a fixed element of $\mathcal{A} \backslash\left\{A_{1}, \ldots, A_{r}\right\}$. We have then $\mathcal{I}\left(\mathcal{A}^{\prime}, \mathcal{T}^{\prime}\right) \leqslant \mathcal{I}(\mathcal{A}, \mathcal{T})+\varepsilon$ and

$$
\operatorname{Dist}\left((\mathcal{A}, \mathcal{T}),\left(\mathcal{A}^{\prime}, \mathcal{T}^{\prime}\right)\right)<\mathcal{L}^{d}\left(\Omega \backslash \bigcup_{1 \leqslant i \leqslant r} A_{i}\right)+\sum_{A \in \mathcal{A} \backslash\left\{A_{1}, \ldots, A_{r}\right\}} \mathcal{L}^{d}(A)<2 \varepsilon .
$$

Hence we need only to consider the case where $\mathcal{A}$ has a finite number of elements.

The main difficulties of the proof are to handle properly the approximation close to $\Gamma$, that is, to push back inside $\Omega$ almost all the interfaces up to a set of $\mathcal{H}^{d-1}$ measure $\varepsilon$, and to keep simultaneously a partition. The essential tools of the proof are the Besicovitch differentiation theorem (Theorem A.1), the Vitali covering theorem (Theorem A.2) and the strong approximation result of Quentin de Gromard (Theorem A.3). Let us summarize the global strategy.

Sketch of the proof. - We fix $\gamma>0$. Since $\mathcal{H}^{d-1}\left(\Gamma \backslash \partial^{*} \Omega\right)=0$, applying an idea of De Giorgi we can find a compact subset $D$ of $\partial^{*} \Omega$ such that $\mathcal{H}^{d-1}(\Gamma \backslash D)<\gamma$ and $D$ is included in an hypersurface. By the definition of the measure theoretic boundary, close to a point of $\partial^{*} \Omega$, the set $\Omega$ looks like a half-space. We cover $D$ by a finite collection of disjoint balls $B\left(x_{i}, r_{i}\right), i \in I_{0} \cup I_{1}$, centered on $D$, whose radii are sufficiently small to ensure that the surface and volume estimates within the balls are controlled by the factor $\gamma$. The remaining part of $\Gamma$ is covered by a finite collection of balls $B\left(y_{s}, s_{j}\right)$, $j \in J_{1}$. The indices of $I_{0}$ correspond to $\Gamma \cap \partial^{*}(\mathcal{A}, \mathcal{T})$ and the indices of $I_{1}$ correspond to $\Gamma \backslash \partial^{*}(\mathcal{A}, \mathcal{T})$. 
We choose $\varepsilon>0$ sufficiently small, depending on the partition $\mathcal{A}$, on $\gamma$ and on the previous families of balls and we apply the strong approximation result of Quentin de Gromard to each set of the partition $\mathcal{A}$. We build then two further family of balls:

- $B\left(x_{i}, r_{i}\right), i \in I_{2}$, cover the interfaces inside $\Omega$, up to a set of $\mathcal{H}^{d-1}$ measure $\varepsilon$.

- $B\left(y_{s}, s_{j}\right), j \in J_{2}$, cover the remaining boundary pieces in $\Omega$.

Inside each ball $B\left(x_{i}, r_{i}\right), i \in I_{0} \cup I_{1} \cup I_{2}$, up to a small fraction, the interfaces are located on hypersurfaces and the radii of the balls are so small that these hypersurfaces are almost flat. Hence we can enclose the interfaces into small flat polyhedral cylinders $D_{i}, i \in I_{0} \cup I_{1} \cup I_{2}$, and by aggregating adequately the cylinders to the sets of the partitions we move these interfaces on the boundaries of these cylinders. The remaining interfaces are enclosed in the balls $B\left(y_{s}, s_{j}\right), j \in J_{1} \cup J_{2}$, and we apply a similar technique, by approximating these balls from the outside by polyhedra.

We have to define delicately the whole process, in order not to lose too much surface energy, and to control the possible interaction between interfaces close to $\Gamma$ and interfaces in $\Omega$. The presence of boundary conditions creates a substantial additional difficulty compared to the polyhedral approximation performed in [14]. Indeed, the most difficult interfaces to handle are those corresponding to $D_{i}, i \in I_{1}$. We first choose the balls $B\left(x_{i}, r_{i}\right), i \in I_{0} \cup I_{1}$, corresponding to $\gamma$. We cover the remaining portion of $\Gamma$ with the balls $B\left(y_{j}, s_{j}\right), j \in J_{1}$. At this point we can already define the cylinders $D_{i}$, $i \in I_{0}$. Then we choose $\varepsilon$ small enough, depending on $\gamma$ and the balls $B\left(x_{i}, r_{i}\right), i \in I_{1}$, to ensure that the perturbation of volume $\varepsilon$ caused when applying Quentin de Gromard's result will not alter significantly the situation inside the balls $B\left(x_{i}, r_{i}\right), i \in I_{1}$. Then we move inside $\Omega$ and we build $D_{i}, i \in I_{2}$. Then we come back to the boundary and we build $D_{i}, i \in I_{1}$. We cover the remaining interfaces in $\Omega$ by the balls $B\left(y_{s}, s_{j}\right), j \in J_{2}$. Finally we aggregate successively each flat polyhedral cylinder to some adequate set of the partition, in two steps, getting first a collection $\mathcal{A}^{\prime}$ and then a second collection $\mathcal{A}^{\prime \prime}$. An ultimate problem is that the collection $\mathcal{A}^{\prime \prime}$ might have overlaps, which is solved by a simple algorithm.

Start of the proof. - Let us consider now an element $(\mathcal{A}, \mathcal{T})$ of $\operatorname{TP}(\Omega)$ such that $\mathcal{A}$ has a finite number of elements $A_{1}, \ldots, A_{h}$. Let $\gamma$ belong to $] 0,1 / 16[$. We start by handling the boundary $\Gamma$, for which we make locally flat approximations controlled by the factor $\gamma$. By hypothesis, $\mathcal{H}^{d-1}\left(\partial_{\Gamma} \bigcup_{m} \Gamma^{m}\right)=0$. Our hypothesis on $\Omega$ implies that $\mathcal{H}^{d-1}\left(\Gamma \backslash \partial^{*} \Omega\right)=0$. We apply first an idea going back to De Giorgi (which is also used at the beginning of the proof of Quentin de Gromard's result). There exists a compact subset $D$ of $\Gamma$ such that $\mathcal{H}^{d-1}(\Gamma \backslash D)<\gamma$ and moreover $D$ is a compact subset of an hypersurface. Using the exterior regularity of $\left.\mathcal{H}^{d-1}\right|_{\Gamma}$, we can find an open set $O_{0}$ containing $(\Gamma \backslash D) \cup \partial_{\Gamma} \bigcup_{m} \Gamma^{m}$ and such that $\mathcal{H}^{d-1}\left(O_{0} \cap \Gamma\right)<\gamma$. We apply Lemma A.4 to the set $\Gamma \backslash O_{0}$ and the hypersurface containing $D$ :

$$
\begin{aligned}
& \exists M_{0} \quad \forall \delta_{0}>0 \quad \exists \eta_{0}>0 \quad \forall x, y \in \Gamma \backslash O_{0} \\
& |x-y|_{2} \leqslant \eta_{0} \Rightarrow d_{2}(y, \tan (\Gamma, x)) \leqslant M_{0} \delta_{0}|x-y|_{2} .
\end{aligned}
$$

Let $\delta_{0}$ in ]0,1/2[ be such that $M_{0} \delta_{0}<\gamma$ and let $\eta_{0}$ be associated to $\delta_{0}$ as in the above property. By Lemma 3.1, for $\mathcal{H}^{d-1}$ almost all $x$ in $\bigcup_{m} \Gamma^{m} \cap \partial^{*}(\mathcal{A}, \mathcal{T}) \backslash O_{0}$,

$$
\lim _{r \rightarrow 0}\left(\alpha_{d-1} r^{d-1}\right)^{-1} \mathcal{H}^{d-1}\left(B(x, r) \cap \partial^{*}(\mathcal{A}, \mathcal{T})\right)=1,
$$




$$
\lim _{r \rightarrow 0}\left(\alpha_{d-1} r^{d-1}\right)^{-1} \int_{B(x, r) \cap \partial^{*}(\mathcal{A}, \mathcal{T})} \tau(\bar{v}(y)) \mathrm{d} \mathcal{H}^{d-1}(y)=\tau(\bar{v}(x)) .
$$

Let $\partial^{* *} \mathcal{A}_{0}$ be the set of the points where the two preceding identities hold simultaneously. For any $x$ in $\partial^{* *} \mathcal{A}_{0}$, there exists a positive $r(x, \gamma)$ such that, for any $r<r(x, \gamma)$,

$$
\begin{array}{r}
\left|\mathcal{H}^{d-1}\left(B(x, r) \cap \partial^{*}(\mathcal{A}, \mathcal{T})\right)-\alpha_{d-1} r^{d-1}\right| \leqslant \gamma \alpha_{d-1} r^{d-1}, \\
\left|\left(\alpha_{d-1} r^{d-1}\right)^{-1} \int_{B(x, r) \cap \partial^{*}(\mathcal{A}, \mathcal{T})} \tau(\bar{v}(y)) \mathrm{d} \mathcal{H}^{d-1}(y)-\tau(\bar{v}(x))\right| \leqslant \gamma .
\end{array}
$$

Next, for $\mathcal{H}^{d-1}$ almost all $x$ in $\Gamma \backslash \partial^{*}(\mathcal{A}, \mathcal{T}) \backslash O_{0}$, there exists a set $A_{x}$ in $\mathcal{A}$ such that $x \in \partial^{*} A_{x}$ and

$$
\lim _{r \rightarrow 0}\left(\alpha_{d} r^{d}\right)^{-1} \mathcal{L}^{d}\left(B(x, r) \cap A_{x}\right)=1 / 2
$$

and moreover

$$
\begin{array}{r}
\lim _{r \rightarrow 0}\left(\alpha_{d} r^{d}\right)^{-1} \mathcal{L}^{d}(B(x, r) \backslash \Omega)=1 / 2, \\
\lim _{r \rightarrow 0}\left(\alpha_{d-1} r^{d-1}\right)^{-1} \mathcal{H}^{d-1}\left(B(x, r) \cap \partial^{*}(\mathcal{A}, \mathcal{T})\right)=0, \\
\lim _{r \rightarrow 0}\left(\alpha_{d-1} r^{d-1}\right)^{-1} \mathcal{H}^{d-1}(B(x, r) \cap \Gamma)=1 .
\end{array}
$$

Let $\partial^{* *} \mathcal{A}_{1}$ be the set of the points where the four preceding identities hold simultaneously. For any $x$ in $\partial^{* *} \mathcal{A}_{1}$, there exists a positive $r(x, \gamma)$ such that, for any $r<r(x, \gamma)$,

$$
\begin{array}{r}
\left|\mathcal{L}^{d}\left(B(x, r) \cap A_{x}\right)-\alpha_{d} r^{d} / 2\right| \leqslant \gamma \alpha_{d} r^{d}, \\
\left|\mathcal{L}^{d}(B(x, r) \backslash \Omega)-\alpha_{d} r^{d} / 2\right| \leqslant \gamma \alpha_{d} r^{d}, \\
\mathcal{H}^{d-1}\left(B(x, r) \cap \partial^{*}(\mathcal{A}, \mathcal{T})\right) \leqslant \gamma \alpha_{d-1} r^{d-1}, \\
\left|\mathcal{H}^{d-1}(B(x, r) \cap \Gamma)-\alpha_{d-1} r^{d-1}\right| \leqslant \gamma \alpha_{d-1} r^{d-1} .
\end{array}
$$

The family of balls $B(x, r), x \in \partial^{* *} \mathcal{A}_{0} \cup \partial^{* *} \mathcal{A}_{1}, r<\min \left(r(x, \gamma), \gamma, \eta_{0}\right)$, is a Vitali relation for

$$
\left(\Gamma \backslash O_{0}\right) \cap\left(\partial^{* *} \mathcal{A}_{0} \cup \partial^{* *} \mathcal{A}_{1}\right) .
$$

By the standard Vitali covering theorem (see Theorem A.2), we may select a finite or countable collection of disjoint balls $B\left(x_{i}, r_{i}\right), i \in I$, such that: for any $i$ in $I, x_{i}$ belongs to the above set, $r_{i}<\min \left(r\left(x_{i}, \gamma\right), \gamma, \eta_{0}\right)$ and

$$
\text { either } \mathcal{H}^{d-1}\left(\Gamma \backslash O_{0} \backslash \bigcup_{i \in I} B\left(x_{i}, r_{i}\right)\right)=0 \quad \text { or } \quad \sum_{i \in I} r_{i}^{d-1}=\infty
$$

Because for each $i$ in $I, r_{i}$ is smaller than $r\left(x_{i}, \gamma\right)$,

$$
\alpha_{d-1}(1-\gamma) \sum_{i \in I} r_{i}^{d-1} \leqslant \mathcal{H}^{d-1}(\Gamma)<\infty,
$$

and therefore the first case occurs, so that we may select two finite subsets $I_{0}, I_{1}$ of $I$ such that 


$$
\begin{aligned}
& \forall i \in I_{0} \quad x_{i} \in \partial^{* *} \mathcal{A}_{0}, \quad \forall i \in I_{1} \quad x_{i} \in \partial^{* *} \mathcal{A}_{1}, \\
& \mathcal{H}^{d-1}\left(\Gamma \backslash O_{0} \backslash \bigcup_{i \in I_{0} \cup I_{1}} B\left(x_{i}, r_{i}\right)\right)<\gamma .
\end{aligned}
$$

Let $i$ belong to $I_{1}$. We have

$$
\begin{aligned}
\mathcal{H}^{d-1} & \left(\Gamma \cap B\left(x_{i}, r_{i}\right) \backslash B\left(x_{i}, r_{i}(1-2 \sqrt{\gamma})\right)\right) \\
& =\mathcal{H}^{d-1}\left(\Gamma \cap B\left(x_{i}, r_{i}\right)\right)-\mathcal{H}^{d-1}\left(\Gamma \cap B\left(x_{i}, r_{i}(1-2 \sqrt{\gamma})\right)\right) \\
& \leqslant(1+\gamma) \alpha_{d-1} r_{i}^{d-1}-(1-\gamma) \alpha_{d-1} r_{i}^{d-1}(1-2 \sqrt{\gamma})^{d-1} \\
& =\alpha_{d-1} r_{i}^{d-1}\left(1+\gamma-(1-\gamma)(1-2 \sqrt{\gamma})^{d-1}\right) \leqslant \alpha_{d-1} r_{i}^{d-1} 2 d \sqrt{\gamma} .
\end{aligned}
$$

Hence

$\sum_{i \in I_{1}} \mathcal{H}^{d-1}\left(\Gamma \cap B\left(x_{i}, r_{i}\right) \backslash B\left(x_{i}, r_{i}(1-2 \sqrt{\gamma})\right)\right) \leqslant 2 d \sqrt{\gamma} \sum_{i \in I_{1}} \alpha_{d-1} r_{i}^{d-1} \leqslant 4 d \sqrt{\gamma} \mathcal{H}^{d-1}(\Gamma)$ and

$$
\mathcal{H}^{d-1}\left(\Gamma \backslash O_{0} \backslash \bigcup_{i \in I_{0}} B\left(x_{i}, r_{i}\right) \backslash \bigcup_{i \in I_{1}} B\left(x_{i}, r_{i}(1-2 \sqrt{\gamma})\right)\right)<\gamma+4 d \sqrt{\gamma} \mathcal{H}^{d-1}(\Gamma)
$$

so that

$$
\mathcal{H}^{d-1}\left(\Gamma \backslash \bigcup_{i \in I_{0}} B\left(x_{i}, r_{i}\right) \backslash \bigcup_{i \in I_{1}} B\left(x_{i}, r_{i}(1-2 \sqrt{\gamma})\right)\right)<2 \gamma+4 d \sqrt{\gamma} \mathcal{H}^{d-1}(\Gamma)
$$

We have a finite number of disjoint closed balls $B\left(x_{i}, r_{i}\right), i \in I_{0}, B\left(x_{i}, r_{i}(1-2 \sqrt{\gamma})\right)$, $i \in I_{1}$. By increasing slightly all the radii $r_{i}$, we can keep the balls disjoint, each $r_{i}$ strictly smaller than $\min \left(r\left(x_{i}, \gamma\right), \gamma, \eta_{0}\right)$ for $i$ in $I_{0} \cup I_{1}$, and get the stronger inequality

$$
\mathcal{H}^{d-1}\left(\Gamma \backslash \bigcup_{i \in I_{0}} \stackrel{\circ}{B}\left(x_{i}, r_{i}\right) \backslash \bigcup_{i \in I_{1}} \stackrel{\circ}{B}\left(x_{i}, r_{i}(1-2 \sqrt{\gamma})\right)\right)<2 \gamma+4 d \sqrt{\gamma} \mathcal{H}^{d-1}(\Gamma)
$$

The above set is a compact subset of $\Gamma$. Using the exterior regularity of $\left.\mathcal{H}^{d-1}\right|_{\Gamma}$, we can find an open set $O_{1}$ such that

$$
\begin{array}{r}
\Gamma \backslash \bigcup_{i \in I_{0}} \stackrel{\circ}{B}\left(x_{i}, r_{i}\right) \backslash \bigcup_{i \in I_{1}} \stackrel{\circ}{B}\left(x_{i}, r_{i}(1-2 \sqrt{\gamma})\right) \subset O_{1}, \\
\mathcal{H}^{d-1}\left(O_{1} \cap \Gamma\right)<3 \gamma+4 d \sqrt{\gamma} \mathcal{H}^{d-1}(\Gamma) .
\end{array}
$$

Let

$$
\rho_{1}=(1 / 6) \operatorname{dist}\left(\Gamma \backslash \bigcup_{i \in I_{0}} \stackrel{\circ}{B}\left(x_{i}, r_{i}\right) \backslash \bigcup_{i \in I_{1}} \stackrel{\circ}{B}\left(x_{i}, r_{i}(1-2 \sqrt{\gamma})\right), \Gamma \backslash O_{1}\right) .
$$

By the definition of the Hausdorff measure $\mathcal{H}^{d-1}$, there exists a collection of balls $B\left(y_{j}, s_{j}\right), j \in J_{1}$, such that:

$\forall j \in J_{1} \quad 0<s_{j}<\rho_{1}, \quad B\left(y_{j}, s_{j}\right) \cap\left(\Gamma \backslash \bigcup_{i \in I_{0}} \stackrel{\circ}{B}\left(x_{i}, r_{i}\right) \backslash \bigcup_{i \in I_{1}} \stackrel{\circ}{B}\left(x_{i}, r_{i}(1-2 \sqrt{\gamma})\right)\right) \neq \emptyset$, 


$$
\begin{gathered}
\sum_{j \in J_{1}} \alpha_{d-1} s_{j}^{d-1}<3 \gamma+4 d \sqrt{\gamma} \mathcal{H}^{d-1}(\Gamma), \\
\Gamma \backslash \bigcup_{i \in I_{0}} \stackrel{B}{B}\left(x_{i}, r_{i}\right) \backslash \bigcup_{i \in I_{1}} \stackrel{\circ}{B}\left(x_{i}, r_{i}(1-2 \sqrt{\gamma})\right) \subset \bigcup_{j \in J_{1}} \stackrel{\circ}{B}\left(y_{j}, s_{j}\right) .
\end{gathered}
$$

By compactness, the set $J_{1}$ can be chosen to be finite. For each $i$ in $I_{0}$, let $P_{i}$ be a convex open polygon inside the hyperplane $\operatorname{hyp}\left(x_{i}, v_{\Gamma}\left(x_{i}\right)\right)$ such that

$$
\begin{gathered}
\operatorname{disc}\left(x_{i}, r_{i}, v_{\Gamma}\left(x_{i}\right)\right) \subset P_{i} \subset \operatorname{disc}\left(x_{i}, r_{i}\left(1+\delta_{0}\right), v_{\Gamma}\left(x_{i}\right)\right), \\
\left|\mathcal{H}^{d-2}\left(\partial P_{i}\right)-\alpha_{d-2} r_{i}^{d-2}\right| \leqslant \delta_{0} \alpha_{d-2} r_{i}^{d-2}, \quad\left|\mathcal{H}^{d-1}\left(P_{i}\right)-\alpha_{d-1} r_{i}^{d-1}\right| \leqslant \delta_{0} \alpha_{d-1} r_{i}^{d-1} .
\end{gathered}
$$

Let $D_{i}$ be the cylinder cyl $\left(P_{i}, M_{0} \delta_{0}\left(1+\delta_{0}\right) r_{i}\right)$ of basis $P_{i}$ and height $2 M_{0} \delta_{0}\left(1+\delta_{0}\right) r_{i}$. Then $\Gamma \cap B\left(x_{i}, r_{i}\right) \subset D_{i}$.

For $i$ in $I_{0} \cup I_{1}$, there exists a unique $l(i)$ in $\{1, \ldots, h\}$ such that $x_{i} \in \partial^{*} A_{l(i)}$. For $i$ in $I_{0}$ there exists a unique integer $m(i)$ such that $x_{i} \in \Gamma^{m(i)}$.

We next deal with the interfaces inside $\Omega$ and we make approximations controlled by the factor $\varepsilon$. We choose $\varepsilon$ sufficiently small compared to $\gamma$ so that, when we perturb the sets by a volume $\varepsilon$, the resulting effect close to the boundary is still of order $\gamma$. We have to delay the approximation of $\Gamma$ by flat interfaces inside the balls $B\left(x_{i}, r_{i}\right), i \in I_{1}$, until we have modified the situation inside $\Omega$.

Let $\varepsilon>0$ be such that $\varepsilon<\gamma$ and

$$
\varepsilon h\left(1+6 \mathcal{H}^{d-1}\left(\partial^{*}(\mathcal{A}, \mathcal{T})\right)\right)<\gamma \alpha_{d} \min _{i \in I_{1}} r_{i}^{d} .
$$

We apply next the approximation result of Quentin de Gromard (see Theorem A.3 in the appendix) to each set $A_{1}, \ldots, A_{h}$ and $\varepsilon$ (here we consider these sets as subsets of $\mathbb{R}^{d}$, i.e., we apply the approximation result in the whole space). For each $i$ in $\{1, \ldots, h\}$, there exists a set $L_{i}$ of finite perimeter, a $C^{1}$ function $f_{i}: \mathbb{R}^{d} \mapsto \mathbb{R}$, a compact set $C_{i}$, an open set $V_{i}$ and an open bounded set $B_{i}$ such that, setting $F_{i}=\left\{x \in \mathbb{R}^{d}: f_{i}(x) \geqslant 0\right\}$, the set $V_{i} \cap \partial F_{i}$ is the hypersurface $\left\{x \in V_{i}: f_{i}(x)=0\right\}$ and

$$
\begin{aligned}
& C_{i} \subset B_{i}, \quad \overline{B_{i}} \subset V_{i} \subset\left\{x \in \mathbb{R}^{d}: \mathrm{d} f_{i}(x) \neq 0\right\}, \quad C_{i} \subset \partial^{*} A_{i} \cap \partial F_{i}, \\
& L_{i} \cap B_{i}=F_{i} \cap B_{i}, \quad V_{i} \cap \partial^{*} F_{i}=V_{i} \cap \partial F_{i}, \\
& \forall x \in C_{i} \quad v_{A_{i}}(x)=v_{F_{i}}(x)=-\left|\mathrm{d} f_{i}(x)\right|^{-1} \mathrm{~d} f_{i}(x), \\
& \mathcal{L}^{d}\left(V_{i}\right)<\varepsilon, \quad \mathcal{L}^{d}\left(A_{i} \Delta L_{i}\right)<\varepsilon, \\
& \mathcal{H}^{d-1}\left(\partial F_{i} \cap\left(V_{i} \backslash C_{i}\right)\right)<\varepsilon, \quad \mathcal{H}^{d-1}\left(\partial^{*} A_{i} \backslash C_{i}\right)<\varepsilon, \quad \mathcal{H}^{d-1}\left(\partial^{*} A_{i} \Delta \partial L_{i}\right)<\varepsilon, \\
& L_{i} \subset \mathcal{V}_{2}\left(A_{i}, \varepsilon\right), \quad \mathbb{R}^{d} \backslash L_{i} \subset \mathcal{V}_{2}\left(\mathbb{R}^{d} \backslash A_{i}, \varepsilon\right) .
\end{aligned}
$$

Since $\left\{A_{1}, \ldots, A_{h}\right\}$ is a partition of $\Omega$ having finite perimeter, then the sets $\partial^{*} A_{i} \cap \partial^{*} A_{j}$, $1 \leqslant i<j \leqslant h$, are pairwise disjoint, and so are the sets $C_{i} \cap C_{j}, 1 \leqslant i<j \leqslant h$. It is possible to impose that the open sets $V_{i}, 1 \leqslant i \leqslant h$, are chosen such that the sets $V_{i} \cap V_{j}, 1 \leqslant i<j \leqslant h$, are also disjoint. More precisely, at the beginning of the proof of Theorem $A .3$, the compact sets $C_{i}, 1 \leqslant i \leqslant h$, are chosen by applying Egoroff Theorem; using the exterior regularity of the measures $\left.\mathcal{H}^{d-1}\right|_{\partial^{*} A_{i}}$, they are then approximated from 
outside by the sets $V_{i}, 1 \leqslant i \leqslant h$. We perform simultaneously this step for all the sets and we impose that each set $V_{i}$ is close enough to $C_{i}$. If we set

$$
r=(1 / 3) \min \left\{d_{2}\left(C_{i} \cap C_{j}, C_{k} \cap C_{l}\right): 1 \leqslant i, j, k, l \leqslant h, i<j, k<l,(i, j) \neq(k, l)\right\},
$$

then $r$ is positive (the sets $C_{i} \cap C_{j}, 1 \leqslant i<j \leqslant h$, are disjoint and compact) and it is enough to require that $V_{i}$ is included in $\mathcal{V}_{2}\left(C_{i}, r\right)$ for each $i$ in $\{1, \ldots, h\}$.

For any $i$ in $\{1, \ldots, h\}$,

$$
\mathcal{H}^{d-1}\left(\Omega \cap \partial L_{i} \backslash C_{i}\right) \leqslant \mathcal{H}^{d-1}\left(\Omega \cap \partial L_{i} \backslash \partial^{*} A_{i}\right)+\mathcal{H}^{d-1}\left(\Omega \cap \partial^{*} A_{i} \backslash C_{i}\right) \leqslant 2 \varepsilon .
$$

Setting $H=\bigcup_{1 \leqslant i \leqslant h}\left(\partial L_{i} \backslash B_{i}\right) \cap \Omega$, we get $\mathcal{H}^{d-1}(H) \leqslant 2 h \varepsilon$. Let next $C=\bigcup_{1 \leqslant i<j \leqslant h} C_{i} \cap$ $C_{j}$. Notice that $C$ is a subset of $\Omega$. We have

$$
\begin{aligned}
\mathcal{H}^{d-1}\left(\Omega \cap \partial L_{i} \backslash C\right) \leqslant & \mathcal{H}^{d-1}\left(\Omega \cap \partial L_{i} \backslash C_{i}\right)+\mathcal{H}^{d-1}\left(\Omega \cap C_{i} \backslash \bigcup_{1 \leqslant j \leqslant h, j \neq i} C_{j}\right) \\
\leqslant & 2 \varepsilon+\mathcal{H}^{d-1}\left(\Omega \cap C_{i} \backslash \bigcup_{1 \leqslant j \leqslant h, j \neq i} \partial^{*} A_{j}\right) \\
& +\mathcal{H}^{d-1}\left(\bigcup_{1 \leqslant j \leqslant h, j \neq i} \Omega \cap \partial^{*} A_{j} \backslash C_{j}\right) \leqslant(h+1) \varepsilon .
\end{aligned}
$$

Setting $G=\bigcup_{1 \leqslant i \leqslant h} \Omega \cap \partial L_{i}$ we get $\mathcal{H}^{d-1}(G \backslash C) \leqslant h(h+1) \varepsilon$. For each $i$ in $\{1, \ldots, h\}$, we apply Lemma A.4 to the set $\partial F_{i} \cap \overline{B_{i}}$ and the hypersurface $\partial F_{i} \cap V_{i}$ (since $B_{i}$ is bounded, then $\partial F_{i} \cap \overline{B_{i}}$ is compact):

$$
\begin{gathered}
\exists M_{i}>0 \quad \forall \delta>0 \quad \exists \eta_{i}>0 \quad \forall x, y \in \partial F_{i} \cap \overline{B_{i}} \\
|x-y|_{2} \leqslant \eta_{i} \Rightarrow d_{2}\left(y, \tan \left(\partial F_{i} \cap V_{i}, x\right)\right) \leqslant M_{i} \delta|x-y|_{2} .
\end{gathered}
$$

For a point $x$ belonging to $C_{i}$, the tangent hyperplane of $\partial F_{i} \cap V_{i}$ at $x$ is precisely hyp $(x, \bar{v}(x))$. Let $M$ be the maximum $\max \left\{1, M_{0}, M_{1}, \ldots, M_{h}\right\}$ and let $\delta$ in $] 0,1 / 2[$ be such that $2 \delta M<\varepsilon$. For $i$ in $\{1, \ldots, h\}$, let $\eta_{i}$ be a positive real number associated to $\delta$ as in the above formula and let $\eta=\min \left\{\eta_{1}, \ldots, \eta_{h}\right\}$. Let also

$$
\begin{aligned}
& \rho_{2}=(1 / 6) \min ( \min \left\{d_{2}\left(C_{i}, \mathbb{R}^{d} \backslash B_{i}\right): 1 \leqslant i \leqslant h\right\}, \\
&\left.\min \left\{d_{2}\left(C_{i} \cap C_{j}, \mathbb{R}^{d} \backslash \Omega\right): 1 \leqslant i<j \leqslant h\right\}\right) .
\end{aligned}
$$

Since each set $C_{i}$ is a compact subset of the open set $B_{i}$ and each set $C_{i} \cap C_{j}$ is a compact subset of $\Omega$, then $\rho_{2}$ is positive.

The map $x \in \partial^{*}(\mathcal{A}, \mathcal{T}) \cap \Omega \mapsto \bar{v}(x) \in P S^{d-1}$ is measurable with respect to $\left.\mathcal{H}^{d-1}\right|_{\partial^{*}}(\mathcal{A}, \mathcal{T}) \cap \Omega$. By Lemma 3.1, for $\mathcal{H}^{d-1}$ almost all $x$ in $\partial^{*}(\mathcal{A}, \mathcal{T}) \cap \Omega$,

$$
\begin{aligned}
\lim _{r \rightarrow 0}\left(\alpha_{d-1} r^{d-1}\right)^{-1} \mathcal{H}^{d-1}\left(B(x, r) \cap \partial^{*}(\mathcal{A}, \mathcal{T})\right) & =1, \\
\lim _{r \rightarrow 0}\left(\alpha_{d-1} r^{d-1}\right)^{-1} \int_{B(x, r) \cap \partial^{*}(\mathcal{A}, \mathcal{T})} \tau(\bar{v}(y)) \mathrm{d} \mathcal{H}^{d-1}(y) & =\tau(\bar{v}(x)) .
\end{aligned}
$$


Let $\partial^{* *} \mathcal{A}$ be the set of the points of $\partial^{*}(\mathcal{A}, \mathcal{T}) \cap \Omega$ where the two preceding identities hold simultaneously. Clearly $\mathcal{H}^{d-1}\left(\partial^{*}(\mathcal{A}, \mathcal{T}) \cap \Omega \backslash \partial^{* *} \mathcal{A}\right)=0$. For any $x$ in $\partial^{* *} \mathcal{A}$, there exists a positive $r(x, \varepsilon)$ such that, for any $r<r(x, \varepsilon)$,

$$
\begin{array}{r}
\left|\mathcal{H}^{d-1}\left(B(x, r) \cap \partial^{*}(\mathcal{A}, \mathcal{T})\right)-\alpha_{d-1} r^{d-1}\right| \leqslant \varepsilon \alpha_{d-1} r^{d-1}, \\
\left|\left(\alpha_{d-1} r^{d-1}\right)^{-1} \int_{B(x, r) \cap \partial^{*}(\mathcal{A}, \mathcal{T})} \tau(\bar{v}(y)) \mathrm{d} \mathcal{H}^{d-1}(y)-\tau(\bar{v}(x))\right| \leqslant \varepsilon .
\end{array}
$$

The family of balls $B(x, r), x \in \partial^{* *} \mathcal{A}, r<\min \left(r(x, \varepsilon), \varepsilon, \eta, \rho_{2}\right)$, is a Vitali relation for $C$. By the standard Vitali covering theorem (see Theorem A.2), we may select a finite or countable collection of disjoint balls $B\left(x_{i}, r_{i}\right), i \in I^{\prime}$, such that: for any $i$ in $I^{\prime}$, $x_{i}$ belongs to $C, r_{i}<\min \left(r\left(x_{i}, \varepsilon\right), \varepsilon, \eta, \rho_{2}\right)$ and

$$
\text { either } \quad \mathcal{H}^{d-1}\left(C \backslash \bigcup_{i \in I^{\prime}} B\left(x_{i}, r_{i}\right)\right)=0 \quad \text { or } \quad \sum_{i \in I^{\prime}} r_{i}^{d-1}=\infty .
$$

Because for each $i$ in $I^{\prime}, r_{i}$ is smaller than $r\left(x_{i}, \varepsilon\right)$,

$$
\alpha_{d-1}(1-\varepsilon) \sum_{i \in I^{\prime}} r_{i}^{d-1} \leqslant \mathcal{H}^{d-1}\left(\partial^{*}(\mathcal{A}, \mathcal{T}) \cap \Omega\right)<\infty,
$$

and therefore the first case occurs, so that we may select a finite subset $I_{2}$ of $I^{\prime}$ such that

$$
\mathcal{H}^{d-1}\left(C \backslash \bigcup_{i \in I_{2}} B\left(x_{i}, r_{i}\right)\right)<\varepsilon .
$$

We have a finite number of disjoint closed balls $B\left(x_{i}, r_{i}\right), i \in I_{2}$. By increasing slightly all the radii $r_{i}$, we can keep the balls disjoint, each $r_{i}$ strictly smaller than $\min \left(r\left(x_{i}, \varepsilon\right), \varepsilon, \eta, \rho_{2}\right)$ for $i$ in $I_{2}$, and get the stronger inequality

$$
\mathcal{H}^{d-1}\left(C \backslash \bigcup_{i \in I_{2}} \stackrel{\circ}{B}\left(x_{i}, r_{i}\right)\right)<\varepsilon .
$$

For each $i$ in $I_{2}$, let $P_{i}$ be a convex open polygon inside the hyperplane hyp $\left(x_{i}, \bar{v}\left(x_{i}\right)\right)$ such that

$$
\begin{gathered}
\operatorname{disc}\left(x_{i}, r_{i}, \bar{v}\left(x_{i}\right)\right) \subset P_{i} \subset \operatorname{disc}\left(x_{i}, r_{i}(1+\delta), \bar{v}\left(x_{i}\right)\right), \\
\left|\mathcal{H}^{d-2}\left(\partial P_{i}\right)-\alpha_{d-2} r_{i}^{d-2}\right| \leqslant \delta \alpha_{d-2} r_{i}^{d-2}, \quad\left|\mathcal{H}^{d-1}\left(P_{i}\right)-\alpha_{d-1} r_{i}^{d-1}\right| \leqslant \delta \alpha_{d-1} r_{i}^{d-1} .
\end{gathered}
$$

We set $\psi=M \delta(1+\delta)$ (hence $\psi<\varepsilon<1$ ). For $i$ in $I_{2}$, let $D_{i}$ be the cylinder $\operatorname{cyl}\left(P_{i}, M \delta(1+\delta) r_{i}\right)$ of basis $P_{i}$ and height $2 \psi r_{i}$. The sets

$$
\bigcup_{i \in I_{2}, x_{i} \in C_{k} \cap C_{l}} D_{i}, \quad 1 \leqslant k<l \leqslant h,
$$

are pairwise disjoint. Indeed, let $i$ be an index in $I_{2}$ such that $x_{i}$ is in $C_{k} \cap C_{l}$; because $r_{i}<\rho_{2}$,

$$
D_{i} \subset B\left(x_{i}, 3 \rho_{2}\right) \subset B_{k} \cap B_{l} \subset V_{k} \cap V_{l}
$$


and the sets $V_{k} \cap V_{l}, 1 \leqslant k<l \leqslant h$, are disjoint. Next, for any $k<l$ in $\{1, \ldots, h\}$, any $i$ in $I_{2}$ such that $x_{i}$ belongs to $C_{k} \cap C_{l}, r_{i}$ is smaller than $\rho_{2}$ and $\eta$, so that $B\left(x_{i}, r_{i}\right) \subset B_{k} \cap B_{l}$,

$$
\begin{array}{r}
\partial L_{k} \cap B\left(x_{i}, r_{i}\right)=\partial F_{k} \cap B\left(x_{i}, r_{i}\right), \quad \partial L_{l} \cap B\left(x_{i}, r_{i}\right)=\partial F_{l} \cap B\left(x_{i}, r_{i}\right), \\
\forall x \in\left(\partial F_{k} \cup \partial F_{l}\right) \cap B\left(x_{i}, r_{i}\right) \quad d_{2}\left(x, \operatorname{hyp}\left(x_{i}, \bar{v}\left(x_{i}\right)\right)\right) \leqslant M \delta\left|x-x_{i}\right|_{2},
\end{array}
$$

whence

$$
\left(\partial L_{k} \cup \partial L_{l}\right) \cap B\left(x_{i}, r_{i}\right) \subset \operatorname{cyl}\left(\operatorname{disc}\left(x_{i}, r_{i}, \bar{v}\left(x_{i}\right)\right), M \delta r_{i}\right) \subset \stackrel{\circ}{D}_{i} .
$$

Next, for $m$ distinct from $k, l$, since $B\left(x_{i}, r_{i}\right) \subset B_{k} \cap B_{l}$ and $B_{k} \cap B_{l} \cap B_{m}=\emptyset$, then

$$
\partial L_{m} \cap B\left(x_{i}, r_{i}\right) \subset \Omega \cap \partial L_{m} \backslash B_{m} \subset H .
$$

Thus

$$
G \cap B\left(x_{i}, r_{i}\right) \subset\left(\partial L_{k} \cup \partial L_{l} \cup H\right) \cap B\left(x_{i}, r_{i}\right) \subset \stackrel{\circ}{D}_{i} \cup H .
$$

We are now ready to perform a first modification of the sets of the partition. The modification consists in pushing all the interfaces in the cylinders $D_{i}, i \in I_{2}$, on one side of the cylinder and forcing the remaining interfaces in $\Omega$ to lie on the boundaries of the sets $L_{1}, \ldots, L_{r}$. We set for $l$ in $\{1, \ldots, h\}$

$$
A_{l}^{\prime}=\left(\Omega \cap L_{l}\right) \cup \bigcup_{1 \leqslant k<l} \bigcup_{\substack{i \in I_{2} \\ x_{i} \in C_{k} \cap C_{l}}} D_{i} \backslash \bigcup_{l<k \leqslant h} \bigcup_{\substack{i \in I_{2} \\ x_{i} \in C_{k} \cap C_{l}}} D_{i},
$$

and $\mathcal{T}^{\prime}\left(A_{l}^{\prime}, m\right)=\mathcal{T}\left(A_{l}, m\right)$ for $m \in \mathbb{N}$. We set also $A_{h+1}^{\prime}=\Omega \backslash \bigcup_{1 \leqslant l \leqslant h} A_{l}^{\prime}$ and

$$
\forall m \in \mathbb{N} \quad \mathcal{T}^{\prime}\left(A_{h+1}^{\prime}, m\right)=1-\sum_{1 \leqslant l \leqslant h} \mathcal{T}\left(A_{l}, m\right) .
$$

Let us show that this new collection approximates correctly the initial partition. For each $l$ in $\{1, \ldots, h\}$,

$$
A_{l}^{\prime} \Delta A_{l} \subset\left(A_{l} \Delta L_{l}\right) \cup \bigcup_{i \in I_{2}} D_{i}
$$

and

$$
A_{h+1}^{\prime} \subset \bigcup_{1 \leqslant l \leqslant h} A_{l}^{\prime} \Delta A_{l}
$$

Thus for $l$ in $\{1, \ldots, h\}$

$$
\mathcal{L}^{d}\left(A_{l}^{\prime} \Delta A_{l}\right) \leqslant \varepsilon+\sum_{i \in I_{2}} 2 \alpha_{d-1} r_{i}^{d-1}(1+\delta) \psi r_{i} .
$$

Yet $r_{i}<\varepsilon$ for $i$ in $I_{2}$ and $\sum_{i \in I_{2}} \alpha_{d-1} r_{i}^{d-1} \leqslant 2 \mathcal{H}^{d-1}\left(\partial^{*}(\mathcal{A}, \mathcal{T}) \cap \Omega\right)$, so that for $l$ in $\{1, \ldots, h\}$

$$
\mathcal{L}^{d}\left(A_{l}^{\prime} \Delta A_{l}\right) \leqslant \varepsilon\left(1+6 \mathcal{H}^{d-1}\left(\partial^{*}(\mathcal{A}, \mathcal{T}) \cap \Omega\right)\right)
$$


Moreover

$$
\mathcal{L}^{d}\left(A_{h+1}^{\prime}\right) \leqslant h \varepsilon\left(1+6 \mathcal{H}^{d-1}\left(\partial^{*}(\mathcal{A}, \mathcal{T}) \cap \Omega\right)\right) .
$$

Two problems remain at this point. First we have now to push the interfaces of $\partial^{*}(\mathcal{A}, \mathcal{T}) \cap \Gamma$ into $\Omega$. Second the sets $A_{1}^{\prime}, \ldots, A_{r}^{\prime}$ might overlap.

We next handle the regions close to $\Gamma$ where the sets of the partition are touching boundary pieces, that is, the family of balls $B\left(x_{i}, r_{i}\right), i \in I_{1}$. We will modify adequately the sets $A_{1}^{\prime}, \ldots, A_{h}^{\prime}$ to ensure that no significant interface is created within these balls. In order to avoid interferences with other interfaces, our modifications will take place inside the balls $B\left(x_{i}, r_{i}\right), i \in I_{1}$. Our technique consists in building a small flat cylinder centered on $\Gamma$ which we add to the set of the partition touching the boundary piece containing $x_{i}$ and which we remove from the other sets of the partition. We have to design carefully this operation in order not to create any significant additional interface. This is the place where we tie together the covering of the boundary and the inner approximation.

Let $i$ belong to $I_{1}$. Because of the condition imposed on $\varepsilon$, we have:

$$
\begin{gathered}
\left|\mathcal{L}^{d}\left(B\left(x_{i}, r_{i}\right) \cap A_{l(i)}^{\prime}\right)-\alpha_{d} r_{i}^{d} / 2\right| \leqslant \gamma \alpha_{d} r_{i}^{d}+\varepsilon \leqslant 2 \gamma \alpha_{d} r_{i}^{d}, \\
\forall l \in\{1, \ldots, h+1\} \backslash\{l(i)\} \quad \mathcal{L}^{d}\left(B\left(x_{i}, r_{i}\right) \cap A_{l}^{\prime}\right) \leqslant 2 \gamma \alpha_{d} r_{i}^{d}+\varepsilon \leqslant 3 \gamma \alpha_{d} r_{i}^{d} .
\end{gathered}
$$

Since in addition

$$
\left|\mathcal{L}^{d}\left(B\left(x_{i}, r_{i}\right) \backslash \Omega\right)-\alpha_{d} r_{i}^{d} / 2\right| \leqslant \gamma \alpha_{d} r_{i}^{d}
$$

it follows that

$$
\mathcal{L}^{d}\left(B\left(x_{i}, r_{i}\right) \cap\left(\Omega \backslash A_{l(i)}^{\prime}\right)\right) \leqslant 3 \gamma \alpha_{d} r_{i}^{d}
$$

Let $P_{i}$ be a convex open polygon inside the hyperplane hyp $\left(x_{i}, v_{\Gamma}\left(x_{i}\right)\right)$ such that

$$
\begin{gathered}
\operatorname{disc}\left(x_{i}, r_{i}(1-2 \sqrt{\gamma}), v_{\Gamma}\left(x_{i}\right)\right) \subset P_{i} \subset \operatorname{disc}\left(x_{i}, r_{i}(1-\sqrt{\gamma}), v_{\Gamma}\left(x_{i}\right)\right), \\
\left|\mathcal{H}^{d-2}\left(\partial P_{i}\right)-\alpha_{d-2} r_{i}^{d-2}(1-\sqrt{\gamma})^{d-2}\right| \leqslant \delta \alpha_{d-2} r_{i}^{d-2}(1-\sqrt{\gamma})^{d-2}, \\
\left|\mathcal{H}^{d-1}\left(P_{i}\right)-\alpha_{d-1} r_{i}^{d-1}(1-\sqrt{\gamma})^{d-1}\right| \leqslant \delta \alpha_{d-1} r_{i}^{d-1}(1-\sqrt{\gamma})^{d-1} .
\end{gathered}
$$

The choice of $\delta_{0}$ guarantees that $M_{0} \delta_{0}\left(1+\delta_{0}\right) r_{i}<2 \gamma r_{i}$. For any $t$ such that

$$
M_{0} \delta_{0}\left(1+\delta_{0}\right) r_{i}<t<\sqrt{\gamma} r_{i}
$$

we have

$$
-t v_{\Gamma}\left(x_{i}\right)+P_{i} \subset B\left(x_{i}, r_{i}\right), \quad \Gamma \cap\left(-t v_{\Gamma}\left(x_{i}\right)+P_{i}\right)=\emptyset .
$$

Moreover $\Gamma \cap B\left(x_{i}, r_{i}(1-2 \sqrt{\gamma})\right) \subset \operatorname{cyl}\left(P_{i}, 2 \gamma r_{i}\right)$ and in addition

$$
\begin{aligned}
\int_{2 \gamma r_{i}<t<\sqrt{\gamma} r_{i}} & \left(\mathcal{H}^{d-1}\left(\left(-t v_{\Gamma}\left(x_{i}\right)+P_{i}\right) \backslash A_{l(i)}^{\prime}\right)\right. \\
& \left.+\sum_{\substack{1 \leqslant l \leqslant h+1 \\
l \neq l(i)}} \mathcal{H}^{d-1}\left(\left(-t v_{\Gamma}\left(x_{i}\right)+P_{i}\right) \cap A_{l}^{\prime}\right)\right) \mathrm{d} t
\end{aligned}
$$




$$
\leqslant \mathcal{L}^{d}\left(B\left(x_{i}, r_{i}\right) \cap\left(\Omega \backslash A_{l(i)}^{\prime}\right)\right)+\sum_{\substack{1 \leqslant l \leqslant h+1 \\ l \neq l(i)}} \mathcal{L}^{d}\left(B\left(x_{i}, r_{i}\right) \cap A_{l}^{\prime}\right) \leqslant 3 \gamma(h+1) \alpha_{d} r_{i}^{d} .
$$

The condition on $\gamma$ yields in particular $\sqrt{\gamma}-2 \gamma \geqslant \sqrt{\gamma} / 2$. Hence there exists $t_{i} \in$ ] $2 \gamma r_{i}, \sqrt{\gamma} r_{i}$ [ such that

$$
\begin{aligned}
& \mathcal{H}^{d-1}\left(\left(-t v_{\Gamma}\left(x_{i}\right)+P_{i}\right) \backslash A_{l(i)}^{\prime}\right)+\sum_{\substack{1 \leqslant l \leqslant h+1 \\
l \neq l(i)}} \mathcal{H}^{d-1}\left(\left(-t v_{\Gamma}\left(x_{i}\right)+P_{i}\right) \cap A_{l}^{\prime}\right) \\
& \quad \leqslant 6 \sqrt{\gamma}(h+1) \alpha_{d} r_{i}^{d-1} .
\end{aligned}
$$

Let $D_{i}$ be the cylinder $D_{i}=\operatorname{cyl}\left(P_{i}, t_{i}\right)$. We deduce from the preceding inequalities that

$$
\begin{aligned}
& \mathcal{H}^{d-1}\left(G \backslash \bigcup_{i \in I_{0} \cup I_{1} \cup I_{2}} \stackrel{\circ}{D}_{i} \backslash \bigcup_{j \in J_{1}} \stackrel{\circ}{B}\left(y_{j}, s_{j}\right)\right) \\
& \leqslant \mathcal{H}^{d-1}\left(G \backslash \bigcup_{i \in I_{2}} \stackrel{\circ}{D}_{i}\right) \\
& \leqslant \mathcal{H}^{d-1}\left(G \backslash \bigcup_{i \in I_{2}} \stackrel{\circ}{B}\left(x_{i}, r_{i}\right)\right)+\mathcal{H}^{d-1}(H) \\
& \leqslant \mathcal{H}^{d-1}(G \backslash C)+\varepsilon+2 h \varepsilon \leqslant h(h+1) \varepsilon+\varepsilon+2 h \varepsilon \leqslant(h+1)(h+2) \varepsilon .
\end{aligned}
$$

Since the sets $\stackrel{\circ}{D}_{i}, i \in I_{0} \cup I_{1}, \stackrel{\circ}{B}\left(y_{j}, s_{j}\right), j \in J_{1}$, cover $\Gamma$, then the set appearing on the left-hand side of the above inequality is compact and it is at positive distance from $\Gamma$. Let

$$
\rho_{3}=(1 / 6) \operatorname{dist}_{L^{1}}\left(\Gamma, G \backslash \bigcup_{i \in I_{0} \cup I_{1} \cup I_{2}} \stackrel{\circ}{D}_{i} \backslash \bigcup_{j \in J_{1}} \stackrel{\circ}{B}\left(y_{j}, s_{j}\right)\right) .
$$

By the definition of the Hausdorff measure $\mathcal{H}^{d-1}$, there exists a collection of balls $B\left(y_{j}, s_{j}\right), j \in J_{2}$, such that:

$$
\begin{gathered}
\forall j \in J_{2} \quad 0<s_{j}<\rho_{3}, \quad B\left(y_{j}, s_{j}\right) \cap\left(G \backslash \bigcup_{i \in I_{0} \cup I_{1} \cup I_{2}} \stackrel{\circ}{D}_{i} \backslash \bigcup_{j \in J_{1}} \stackrel{\circ}{B}\left(y_{j}, s_{j}\right)\right) \neq \emptyset, \\
G \backslash \bigcup_{i \in I_{0} \cup I_{1} \cup I_{2}} \stackrel{\circ}{D_{i} \backslash \bigcup} \bigcup_{j \in J_{1}} \stackrel{\circ}{B}\left(y_{j}, s_{j}\right) \subset \bigcup_{j \in J_{2}} \stackrel{\circ}{B}\left(y_{j}, s_{j}\right), \\
\\
\sum_{j \in J_{2}} \alpha_{d-1} s_{j}^{d-1} \leqslant(h+1)(h+2) \varepsilon+\varepsilon .
\end{gathered}
$$

By compactness, we might assume in addition that $J_{2}$ is finite.

For each $j$ in $J_{1} \cup J_{2}$, let $Q_{j}$ be an open convex polyhedral set such that $B\left(y_{j}, s_{j}\right) \subset$ $Q_{j} \subset B\left(y_{j}, 2 s_{j}\right)$ and $\mathcal{H}^{d-1}\left(\partial Q_{j}\right) \leqslant \alpha_{d-1} 2^{d-1} s_{j}^{d-1}$.

We now perform the second modification. The resulting sets will be polyhedral and all the interfaces up to a small portion are pushed back into $\Omega$. We set for $l$ in $\{1, \ldots, h\}$

$$
A_{l}^{\prime \prime}=A_{l}^{\prime} \cup\left(\bigcup_{\substack{i \in I_{1} \\ l(i)=l}} D_{i} \cap \Omega\right) \cup\left(\bigcup_{\substack{m \in \mathbb{N} \\ \mathcal{T}\left(A_{l}, m\right)=1}} \bigcup_{\substack{i \in I_{0} \\ x_{i} \in \Gamma^{m}}} D_{i} \cap \Omega\right)
$$




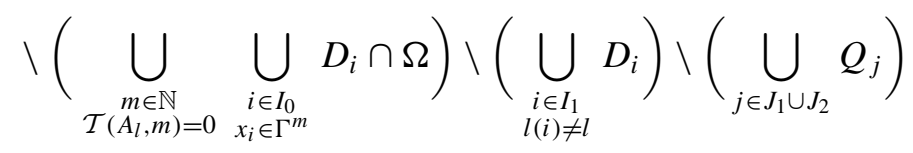

and $\mathcal{T}^{\prime \prime}\left(A_{l}^{\prime \prime}, m\right)=\mathcal{T}\left(A_{l}, m\right)$ for $m \in \mathbb{N}$. We set also $A_{h+1}^{\prime \prime}=\Omega \backslash \bigcup_{1 \leqslant l \leqslant h} A_{l}^{\prime \prime}$ and

$$
\forall m \in \mathbb{N} \quad \mathcal{T}^{\prime \prime}\left(A_{h+1}^{\prime \prime}, m\right)=1-\sum_{1 \leqslant l \leqslant h} \mathcal{T}\left(A_{l}, m\right) .
$$

We first check that the collection $A_{1}^{\prime \prime}, \ldots, A_{h+1}^{\prime \prime}$ approximates the initial partition with respect to the metric Dist. For each $l$ in $\{1, \ldots, h\}$,

$$
A_{l}^{\prime \prime} \Delta A_{l} \subset\left(A_{l}^{\prime} \Delta A_{l}\right) \cup \bigcup_{i \in I_{0} \cup I_{1} \cup I_{2}} D_{i} \cup \bigcup_{j \in J_{1} \cup J_{2}} Q_{j},
$$

whence

$$
\begin{aligned}
\mathcal{L}^{d}\left(A_{l}^{\prime \prime} \Delta A_{l}\right) \leqslant & \varepsilon\left(1+6 \mathcal{H}^{d-1}\left(\partial^{*}(\mathcal{A}, \mathcal{T}) \cap \Omega\right)\right)+\sum_{i \in I_{0}} 2 \alpha_{d-1} r_{i}^{d-1}\left(1+\delta_{0}\right)^{2} \gamma r_{i} \\
& +\sum_{i \in I_{1}} 2 \alpha_{d-1} r_{i}^{d-1}(1+\delta) \sqrt{\gamma} r_{i}+\sum_{i \in I_{2}} 2 \alpha_{d-1} r_{i}^{d-1}(1+\delta) \psi r_{i} \\
& +\sum_{j \in J_{1} \cup J_{2}} \alpha_{d}\left(2 s_{j}\right)^{d} .
\end{aligned}
$$

Yet each $r_{i}$ is smaller than $\gamma$,

$$
\begin{aligned}
\sum_{i \in I_{0} \cup I_{1}} \alpha_{d-1} r_{i}^{d-1} & \leqslant 2 \mathcal{H}^{d-1}(\Gamma), \\
\sum_{i \in I_{2}} \alpha_{d-1} r_{i}^{d-1} & \leqslant 2 \mathcal{H}^{d-1}\left(\partial^{*}(\mathcal{A}, \mathcal{T}) \cap \Omega\right), \\
\sum_{j \in J_{1} \cup J_{2}} \alpha_{d-1} s_{j}^{d-1} & \leqslant 3 \gamma+4 d \sqrt{\gamma} \mathcal{H}^{d-1}(\Gamma)+(h+1)(h+2) \varepsilon+\varepsilon,
\end{aligned}
$$

so that for $l$ in $\{1, \ldots, h\}$

$$
\begin{aligned}
\mathcal{L}^{d}\left(A_{l}^{\prime \prime} \Delta A_{l}\right) \leqslant & \varepsilon\left(1+6 \mathcal{H}^{d-1}\left(\partial^{*}(\mathcal{A}, \mathcal{T}) \cap \Omega\right)\right)+\gamma\left(9 \mathcal{H}^{d-1}(\Gamma)+6 \mathcal{H}^{d-1}\left(\partial^{*}(\mathcal{A}, \mathcal{T}) \cap \Omega\right)\right) \\
& +2^{d}\left(3 \gamma+4 d \sqrt{\gamma} \mathcal{H}^{d-1}(\Gamma)+(h+1)(h+2) \varepsilon+\varepsilon\right) .
\end{aligned}
$$

Moreover

$$
A_{h+1}^{\prime \prime} \subset \bigcup_{1 \leqslant l \leqslant h}\left(A_{l} \backslash L_{l}\right) \cup \bigcup_{i \in I_{0} \cup I_{1}} D_{i} \cup \bigcup_{j \in J_{1} \cup J_{2}} Q_{j},
$$

whence

$$
\mathcal{L}^{d}\left(A_{h+1}^{\prime \prime}\right) \leqslant h \varepsilon+9 \gamma \mathcal{H}^{d-1}(\Gamma)+2^{d}\left(3 \gamma+4 d \sqrt{\gamma} \mathcal{H}^{d-1}(\Gamma)+(h+1)(h+2) \varepsilon+\varepsilon\right) .
$$

It follows that

$$
\begin{aligned}
\operatorname{Dist}\left(\mathcal{A}, \mathcal{A}^{\prime \prime}\right) \leqslant & h(6 \varepsilon+6 \gamma) \mathcal{H}^{d-1}\left(\partial^{*}(\mathcal{A}, \mathcal{T}) \cap \Omega\right)+(h+1)\left(9 \gamma+2^{d+2} d \sqrt{\gamma}\right) \mathcal{H}^{d-1}(\Gamma) \\
& +2^{d} 3(h+1) \gamma+h \varepsilon+2^{d}(h+1)((h+1)(h+3) \varepsilon+\varepsilon),
\end{aligned}
$$


and the collection $\mathcal{A}^{\prime \prime}$ approximates the collection $\mathcal{A}$ with respect to the metric Dist.

We show next that $\mathcal{A}^{\prime \prime}$ is polyhedral. The sets $Q_{j}, j \in J_{1} \cup J_{2}, \stackrel{\circ}{D}_{i}, i \in I_{0} \cup I_{1} \cup I_{2}$ cover $G$. The definition of the sets $A_{m}^{\prime \prime}, 1 \leqslant m \leqslant h+1$, implies that

$$
\begin{aligned}
& \bigcup_{1 \leqslant m \leqslant h+1} \partial A_{m}^{\prime \prime} \subset \Gamma \cup\left(G \backslash\left(\bigcup_{i \in I_{0} \cup I_{1} \cup I_{2}} \stackrel{\circ}{D}_{i} \cup \bigcup_{j \in J_{1} \cup J_{2}} \stackrel{\circ}{Q}_{j}\right)\right) \cup \bigcup_{i \in I_{0} \cup I_{1} \cup I_{2}} \partial D_{i} \cup \bigcup_{j \in J_{1} \cup J_{2}} \partial Q_{j} \\
& \quad=\Gamma \cup \bigcup_{i \in I_{0} \cup I_{1} \cup I_{2}} \partial D_{i} \cup \bigcup_{j \in J_{1} \cup J_{2}} \partial Q_{j}
\end{aligned}
$$

and the collection $\mathcal{A}^{\prime \prime}$ is polyhedral. We next refine the above inclusion in order to estimate the surface energy of $\mathcal{A}^{\prime \prime}$. Let us again consider $k<l$ in $\{1, \ldots, h\}$ and $i$ in $I_{2}$ such that $x_{i}$ belongs to $C_{k} \cap C_{l}$. Let $\beta=\sqrt{1-\psi^{2}}$. We set

$$
G_{i}=\operatorname{disc}\left(x_{i}-\psi r_{i} v_{L_{k}}\left(x_{i}\right), \beta r_{i}, \bar{v}\left(x_{i}\right)\right)=\operatorname{disc}\left(x_{i}+\psi r_{i} v_{L_{l}}\left(x_{i}\right), \beta r_{i}, \bar{v}\left(x_{i}\right)\right) .
$$

We claim that $G_{i}$ is included in the interior of $L_{k}$ and in the interior of $\mathbb{R}^{d} \backslash L_{l}$. Indeed, $G_{i}$ is included in $B\left(x_{i}, r_{i}\right) \cap \partial D_{i}$ and therefore $G_{i}$ does not intersect $\partial L_{k} \cup \partial L_{l}$. Since $v_{L_{k}}\left(x_{i}\right)=v_{A_{k}}\left(x_{i}\right)=-v_{L_{l}}\left(x_{i}\right)=-v_{A_{l}}\left(x_{i}\right)$ is the exterior normal vector to $L_{k}$ at $x_{i}$ and the interior normal vector to $L_{l}$ at $x_{i}$ then $G_{i}$ is included in $\stackrel{\circ}{L}_{k} \backslash \bar{L}_{l}$. The sets

$$
\bigcup_{i \in I_{2}, x_{i} \in C_{k^{\prime}} \cap C_{l^{\prime}}} D_{i}, \quad 1 \leqslant k^{\prime}<l^{\prime} \leqslant h,
$$

being closed and disjoint, looking at the definition of $A_{k}^{\prime}$ and $A_{l}^{\prime}$, we see that for a sufficiently small neighborhood $W_{i}$ of $G_{i}$

$$
W_{i} \cap A_{k}^{\prime}=W_{i} \cap L_{k}, \quad W_{i} \cap A_{l}^{\prime}=W_{i} \cap L_{l}=\emptyset,
$$

whence $\partial A_{k}^{\prime} \cap G_{i}=\partial A_{l}^{\prime} \cap G_{i}=\emptyset$. The definition of the sets $A_{m}^{\prime \prime}, 1 \leqslant m \leqslant h+1$, implies that

$$
\partial A_{k}^{\prime \prime} \cap G_{i} \subset \bigcup_{j \in J_{1} \cup J_{2}} \partial Q_{j}, \quad \partial A_{l}^{\prime \prime} \cap G_{i}=\emptyset .
$$

It follows that

$$
\begin{aligned}
\partial^{*}\left(\mathcal{A}^{\prime \prime}, \mathcal{T}^{\prime \prime}\right) \cap \Omega \subset \bigcup_{i \in I_{0}}\left(\partial D_{i} \cap \Omega\right) \cup \bigcup_{i \in I_{1}}\left(\left(\partial D_{i} \cap \Omega\right) \cap\left(\left(\mathbb{R}^{d} \backslash A_{l(i)}^{\prime}\right) \cup \bigcup_{l \neq l(i)} A_{l}^{\prime}\right)\right) \\
\cup \bigcup_{i \in I_{2}}\left(\partial D_{i} \backslash G_{i}\right) \cup \bigcup_{j \in J_{1} \cup J_{2}} \partial Q_{j} .
\end{aligned}
$$

Notice also that $\stackrel{\circ}{D}_{i}, i \in I_{0} \cup I_{1}$ and $B\left(y_{j}, s_{j}\right), j \in J_{1}$ cover $\Gamma$, therefore

$$
\overline{\partial^{*}\left(\mathcal{A}^{\prime \prime}, \mathcal{T}^{\prime \prime}\right)} \cap \Gamma \subset \bigcup_{i \in I_{0} \cup I_{1}}\left(\partial D_{i} \cap \Gamma\right) \cup \bigcup_{j \in J_{1}}\left(\bar{Q}_{j} \cap \Gamma\right) \subset \bigcup_{i \in I_{0} \cup I_{1}}\left(\partial D_{i} \cap \Gamma\right) \cup\left(O_{1} \cap \Gamma\right) .
$$

Notice that for $i$ in $I_{0} \cup I_{1}$, the intersection $\partial D_{i} \cap \Gamma$ is contained in the "lateral" part of the cylinder $D_{i}$. Therefore 


$$
\begin{aligned}
& \mathcal{H}^{d-1}\left(\overline{\partial^{*}\left(\mathcal{A}^{\prime \prime}, \mathcal{T}^{\prime \prime}\right)} \cap \Gamma\right) \\
& \quad \leqslant \sum_{i \in I_{0} \cup I_{1}} \mathcal{H}^{d-1}\left(\partial D_{i} \cap \Gamma\right)+\mathcal{H}^{d-1}\left(O_{1} \cap \Gamma\right) \\
& \quad \leqslant \sum_{i \in I_{0}} 2 \gamma \alpha_{d-2} r_{i}^{d-1}\left(1+\delta_{0}\right)+\sum_{i \in I_{1}} 2 \sqrt{\gamma} \alpha_{d-2} r_{i}^{d-1}\left(1+\delta_{0}\right)+3 \gamma+4 d \sqrt{\gamma} \mathcal{H}^{d-1}(\Gamma) \\
& \quad \leqslant 6 \sqrt{\gamma}\left(\alpha_{d-2} / \alpha_{d-1}\right) \mathcal{H}^{d-1}(\Gamma)+3 \gamma+4 d \sqrt{\gamma} \mathcal{H}^{d-1}(\Gamma) .
\end{aligned}
$$

Finally

$$
\begin{aligned}
\mathcal{I}\left(\mathcal{A}^{\prime \prime}, \mathcal{T}^{\prime \prime}\right) \leqslant & \sum_{i \in I_{0}} \int_{\partial D_{i} \cap \Omega} \tau(\bar{v}(x)) \mathrm{d} \mathcal{H}^{d-1}(x)+\sum_{i \in I_{1}} \int_{\Delta} \tau(\bar{v}(x)) \mathrm{d} \mathcal{H}^{d-1}(x) \\
& +\sum_{i \in I_{2}} \int_{\partial D_{i} \backslash G_{i}} \tau(\bar{v}(x)) \mathrm{d} \mathcal{H}^{d-1}(x)+\tau_{\max } \sum_{j \in J_{1} \cup J_{2}} \mathcal{H}^{d-1}\left(\partial Q_{j}\right) \\
& +\tau_{\max } \mathcal{H}^{d-1}\left(\partial^{*}\left(\mathcal{A}^{\prime \prime}, \mathcal{T}^{\prime \prime}\right) \cap \Gamma\right),
\end{aligned}
$$

where the set of integration for the second integral is $\Delta=\left(\partial D_{i} \cap \Omega \backslash A_{l(i)}^{\prime}\right) \cup$ $\left(\partial D_{i} \cap \bigcup_{l \neq l(i)} A_{l}^{\prime}\right)$. We use now the various estimates obtained in the course of the approximation. We get

$$
\begin{aligned}
\mathcal{I}\left(\mathcal{A}^{\prime \prime}, \mathcal{T}^{\prime \prime}\right) \leqslant & \sum_{i \in I_{0}} \alpha_{d-1} r_{i}^{d-1}(1+\delta) \tau\left(\bar{v}\left(x_{i}\right)\right)+\sum_{i \in I_{0}} \tau_{\max } \alpha_{d-2} r_{i}^{d-1} 2 M_{0} \delta_{0}\left(1+\delta_{0}\right)^{2} \\
& +\sum_{i \in I_{1}} \tau_{\max }\left(6 \sqrt{\gamma}(h+1) \alpha_{d} r_{i}^{d-1}+2 \sqrt{\gamma}(1+\delta) \alpha_{d-2} r_{i}^{d-1}\right) \\
& +\sum_{i \in I_{2}} \alpha_{d-1} r_{i}^{d-1}(1+\delta) \tau\left(\bar{v}\left(x_{i}\right)\right)+\sum_{i \in I_{2}} \tau_{\max } \alpha_{d-1} r_{i}^{d-1}\left(4 \psi+1+\delta-\beta^{d-1}\right) \\
& +\sum_{j \in J_{1} \cup J_{2}} \tau_{\max } \alpha_{d-1} 2^{d-1} s_{j}^{d-1} \\
\leqslant & \frac{1+\delta}{1-\gamma} \sum_{i \in I_{0} \cup I_{2}} \quad \int_{B\left(x_{i}, r_{i}\right) \cap \partial^{*}(\mathcal{A}, \mathcal{T})} \tau(\bar{v}(y)) \mathrm{d} \mathcal{H}^{d-1}(y) \\
& +2 \tau_{\max } \mathcal{H}^{d-1}(\Gamma)\left(5 \gamma \alpha_{d-2} / \alpha_{d-1}+6 \sqrt{\gamma}(h+1) \alpha_{d} / \alpha_{d-1}\right. \\
& \left.+3 \sqrt{\gamma} \alpha_{d-2} / \alpha_{d-1}\right) \\
& +2 \tau_{\max } \mathcal{H}^{d-1}\left(\partial^{*}(\mathcal{A}, \mathcal{T}) \cap \Omega\right)\left(4 \psi+(1+\delta)-\left(1-\psi^{2}\right)^{d-1}\right) \\
& +\tau_{\max } 2^{d-1}\left(3 \gamma+4 d \sqrt{\gamma} \mathcal{H}^{d-1}(\Gamma)+(h+1)(h+2) \varepsilon+\varepsilon\right) \\
\leqslant & \left((1+\delta) /(1-\gamma)+2\left(\tau_{\max } / \tau_{\min }\right)((d+3) \varepsilon+\delta)\right) \mathcal{I}(\mathcal{A}, \mathcal{T}) \\
& +16 \tau_{\max } \mathcal{H}^{d-1}(\Gamma) \sqrt{\gamma}\left(\alpha_{d-2} / \alpha_{d-1}+(h+1) \alpha_{d} / \alpha_{d-1}\right) \\
& +\tau_{\max } 2^{d-1}\left(3 \gamma+4 d \sqrt{\gamma} \mathcal{H}^{d-1}(\Gamma)+(h+1)(h+2) \varepsilon+\varepsilon\right)
\end{aligned}
$$

where we have used the inequality $\psi<\varepsilon$ in the last step. The only remaining problem is that the sets of the collection $\mathcal{A}^{\prime \prime}$ are not necessarily disjoint. This issue is solved through the next lemma. We recall that a subset $A$ of $\Omega$ is said to be polyhedral if $\partial^{*} A \cap \Omega$ is included in the union of a finite number of hyperplanes. 
LEMMA 3.6. - Let $\mathcal{A}$ be a finite collection of bounded polyhedral subsets of $\Omega$ and let $\mathcal{T}$ be a touching function associated to $\mathcal{A}$. There exists a finite collection $\mathcal{B}$ of bounded polyhedral subsets of $\Omega$ and an associated touching function $\mathcal{S}$ such that

$$
\begin{gathered}
\bigcup_{B \in \mathcal{B}} B=\bigcup_{A \in \mathcal{A}} A, \quad \mathcal{I}(\mathcal{B}, \mathcal{S}) \leqslant \mathcal{I}(\mathcal{A}, \mathcal{T}), \quad \mathcal{L}^{d}\left(\bigcup_{B_{1}, B_{2} \in \mathcal{B}, B_{1} \neq B_{2}} B_{1} \cap B_{2}\right)=0, \\
\operatorname{Dist}((\mathcal{B}, \mathcal{S}),(\mathcal{A}, \mathcal{T})) \leqslant(|\mathcal{A}|-1)|\mathcal{A}| \mathcal{L}^{d}\left(\bigcup_{A_{1}, A_{2} \in \mathcal{A}, A_{1} \neq A_{2}} A_{1} \cap A_{2}\right) .
\end{gathered}
$$

Remark. - The collection $\mathcal{B}$ is not necessarily a partition. To define its surface energy $\mathcal{I}(\mathcal{B}, \mathcal{S})$, we simply use the first formula provided for $\mathcal{I}$ at the beginning of Section 3 . We do the same to define the distance Dist between $(\mathcal{B}, \mathcal{S})$ and $(\mathcal{A}, \mathcal{T})$ : the definition given for partitions is readily extended to more general collections of sets.

Proof of Theorem 3.5 continued. - We apply Lemma 3.6 to $\left(\mathcal{A}^{\prime \prime}, \mathcal{T}^{\prime \prime}\right)$ to get a finite collection $\left(\mathcal{A}^{\prime \prime \prime}, \mathcal{T}^{\prime \prime \prime}\right)$ of polyhedral subsets of $\Omega$ with touching function $\mathcal{T}^{\prime \prime \prime}$. Because of the choice of $A_{h+1}^{\prime \prime}$, we have $\bigcup_{A \in \mathcal{A}^{\prime \prime}}=\Omega$. Moreover

$$
\begin{aligned}
\mathcal{L}^{d}\left(\bigcup_{A_{1}, A_{2} \in \mathcal{A}^{\prime \prime}, A_{1} \neq A_{2}} A_{1} \cap A_{2}\right) & \leqslant \sum_{1 \leqslant k<l \leqslant h} \mathcal{L}^{d}\left(A_{k}^{\prime \prime} \cap A_{l}^{\prime \prime}\right) \\
& \leqslant \sum_{1 \leqslant k<l \leqslant h} \mathcal{L}^{d}\left(A_{k}^{\prime \prime} \Delta A_{k}\right)+\mathcal{L}^{d}\left(A_{l}^{\prime \prime} \Delta A_{l}\right) .
\end{aligned}
$$

Therefore the resulting collection $\mathcal{A}^{\prime \prime \prime}$ is a polyhedral element of $\operatorname{TP}(\Omega)$ satisfying

$$
\begin{aligned}
\operatorname{Dist}\left(\left(\mathcal{A}^{\prime \prime \prime}, \mathcal{T}^{\prime \prime \prime}\right),(\mathcal{A}, \mathcal{T})\right) \leqslant & 3 h(6 \varepsilon+6 \gamma) \mathcal{I}(\mathcal{A}, \mathcal{T}) / \tau_{\min } \\
& +3(h+1)\left(9 \gamma+2^{d+2} d \sqrt{\gamma}\right) \mathcal{H}^{d-1}(\Gamma)+2^{d} 9(h+1) \gamma \\
& +3 h \varepsilon+2^{d} 3(h+1)((h+1)(h+3) \varepsilon+\varepsilon)
\end{aligned}
$$

and furthermore

$$
\begin{aligned}
\mathcal{I}\left(\mathcal{A}^{\prime \prime \prime}, \mathcal{T}^{\prime \prime \prime}\right) \leqslant \mathcal{I}\left(\mathcal{A}^{\prime \prime}, \mathcal{T}^{\prime \prime}\right) \leqslant & \left((1+\delta) /(1-\gamma)+2\left(\tau_{\max } / \tau_{\min }\right)((d+3) \varepsilon+\delta)\right) \mathcal{I}(\mathcal{A}, \mathcal{T}) \\
& +16 \tau_{\max } \mathcal{H}^{d-1}(\Gamma) \sqrt{\gamma}\left(\alpha_{d-2} / \alpha_{d-1}+(h+1) \alpha_{d} / \alpha_{d-1}\right) \\
& +\tau_{\max } 2^{d-1}\left(3 \gamma+4 d \sqrt{\gamma} \mathcal{H}^{d-1}(\Gamma)+(h+1)(h+2) \varepsilon+\varepsilon\right) .
\end{aligned}
$$

Since $\mathcal{I}(\mathcal{A}, \mathcal{T})$ is finite and $h, \mathcal{H}^{d-1}(\Gamma), \tau_{\max }, \tau_{\min }$ are fixed, we have the required approximation by choosing $\gamma, \delta, \varepsilon$ sufficiently small.

Proof of Lemma 3.6. - We use an algorithm to build $(\mathcal{B}, \mathcal{S})$ starting from $(\mathcal{A}, \mathcal{T})$. We define for $A$ a subset of $\Omega$ having finite perimeter and $T$ a function of $\mathcal{F}$

$$
\mathcal{I}(A, T)=\frac{1}{2} \int_{\partial^{*} A \cap \Omega} \tau\left(v_{A}(x)\right) \mathrm{d} \mathcal{H}^{d-1}(x)+\sum_{m \in \mathbb{N}}(1-T(m)) \int_{\partial^{*} A \cap \Gamma^{m}} \tau\left(v_{A}(x)\right) \mathrm{d} \mathcal{H}^{d-1}(x)
$$

so that the surface energy of a collection with touching status $(\mathcal{A}, \mathcal{T})$ can be expressed as

$$
\mathcal{I}(\mathcal{A}, \mathcal{T})=\sum_{A \in \mathcal{A}} \mathcal{I}(A, \mathcal{T}(A, \cdot))
$$


We initialize the algorithm with the collection $\mathcal{B}^{0}=\mathcal{A}$ and the touching function $\mathcal{S}^{0}=\mathcal{T}$. We describe next the $k$-step of the algorithm. Suppose that we have built the collection $\mathcal{B}^{k}$ and the function $\mathcal{S}^{k}$ for some $k$ in $\mathbb{N}$. If

$$
\mathcal{L}^{d}\left(\bigcup_{B_{1}, B_{2} \in \mathcal{B}^{k}, B_{1} \neq B_{2}} B_{1} \cap B_{2}\right)=0
$$

the algorithm stops. Otherwise, let $B_{1}, B_{2}$ be two sets of $\mathcal{B}^{k}$ such that $\mathcal{L}^{d}\left(B_{1} \cap B_{2}\right)>0$. Let $B_{1}^{\prime}=B_{1} \backslash B_{2}$ and $B_{2}^{\prime}=B_{2} \backslash B_{1}$. We have

$$
\max \left(\mathcal{L}^{d}\left(B_{1}^{\prime} \Delta B_{1}\right), \mathcal{L}^{d}\left(B_{2}^{\prime} \Delta B_{2}\right)\right) \leqslant \mathcal{L}^{d}\left(B_{1} \cap B_{2}\right) \leqslant \mathcal{L}^{d}\left(\bigcup_{B_{1}, B_{2} \in \mathcal{B}^{k}, B_{1} \neq B_{2}} B_{1} \cap B_{2}\right) .
$$

Moreover

$$
v_{B_{1} \backslash B_{2}}(x)= \begin{cases}v_{B_{1}}(x) & \text { if } x \in \partial^{*} B_{1} \backslash \overline{B_{2}}, \\ -v_{B_{2}}(x) & \text { if } x \in \partial^{*} B_{2} \cap \stackrel{\circ}{B}_{1}, \\ v_{B_{1}}(x) & \text { if } x \in \partial^{*} B_{2} \cap \partial^{*} B_{1} \text { and } v_{B_{1}}(x)+v_{B_{2}}(x)=0, \\ 0 & \text { elsewhere. }\end{cases}
$$

This result is quite direct here because we deal only with polyhedral sets. See [53] for a more general result. Using the symmetry and the positivity of $\tau$,

$$
\begin{aligned}
\mathcal{I}\left(B_{1}^{\prime}, \mathcal{S}^{k}\left(B_{1}, \cdot\right)\right) \leqslant & \frac{1}{2} \int_{\Omega \cap \partial^{*} B_{1} \backslash \overline{B_{2}}} \tau\left(v_{B_{1}}(x)\right) \mathrm{d} \mathcal{H}^{d-1}(x)+\frac{1}{2} \int_{B_{1} \cap \partial^{*} B_{2}} \tau\left(v_{B_{2}}(x)\right) \mathrm{d} \mathcal{H}^{d-1}(x) \\
& +\frac{1}{2} \int_{\partial^{*} B_{1} \cap \partial^{*} B_{2}} \tau\left(v_{B_{1}}(x)\right) \mathrm{d} \mathcal{H}^{d-1}(x) \\
& +\sum_{m \in \mathbb{N}}\left(1-\mathcal{S}^{k}\left(B_{1}, m\right)\right) \int_{\Gamma \cap \partial^{*} B_{1} \backslash \overline{B_{2}}} \tau\left(v_{B_{1}}(x)\right) \mathrm{d} \mathcal{H}^{d-1}(x), \\
\mathcal{I}\left(B_{2}^{\prime}, \mathcal{S}^{k}\left(B_{2}, \cdot\right)\right) \leqslant & \frac{1}{2} \int_{\Omega \cap \partial^{*} B_{2} \backslash \overline{B_{1}}} \tau\left(v_{B_{2}}(x)\right) \mathrm{d} \mathcal{H}^{d-1}(x)+\frac{1}{2} \int_{\dot{B}_{2} \cap \partial^{*} B_{1}} \tau\left(v_{B_{1}}(x)\right) \mathrm{d} \mathcal{H}^{d-1}(x) \\
& +\frac{1}{2} \int_{\partial^{*} B_{2} \cap \partial^{*} B_{1}} \tau\left(v_{B_{2}}(x)\right) \mathrm{d} \mathcal{H}^{d-1}(x) \\
& +\sum_{m \in \mathbb{N}}\left(1-\mathcal{S}^{k}\left(B_{2}, m\right)\right) \int_{\Gamma \cap \partial^{*} B_{2} \backslash \overline{B_{1}}} \tau\left(v_{B_{2}}(x)\right) \mathrm{d} \mathcal{H}^{d-1}(x) .
\end{aligned}
$$

Summing the two inequalities yields

$$
\mathcal{I}\left(B_{1}^{\prime}, \mathcal{S}^{k}\left(B_{1}, \cdot\right)\right)+\mathcal{I}\left(B_{2}^{\prime}, \mathcal{S}^{k}\left(B_{2}, \cdot\right)\right) \leqslant \mathcal{I}\left(B_{1}, \mathcal{S}^{k}\left(B_{1}, \cdot\right)\right)+\mathcal{I}\left(B_{2}, \mathcal{S}^{k}\left(B_{2}, \cdot\right)\right) .
$$

Two cases can occur. 
- If $\mathcal{I}\left(B_{1}^{\prime}, \mathcal{S}^{k}\left(B_{1}, \cdot\right)\right) \leqslant \mathcal{I}\left(B_{1}, \mathcal{S}^{k}\left(B_{1}, \cdot\right)\right)$, then we set

$$
\begin{aligned}
\mathcal{B}^{k+1}= & \left\{B_{1}^{\prime}\right\} \cup \mathcal{B}^{k} \backslash\left\{B_{1}\right\}, \quad \mathcal{S}^{k+1}\left(B_{1}^{\prime}, \cdot\right)=\mathcal{S}^{k}\left(B_{1}, \cdot\right), \\
& \forall B \in \mathcal{B}^{k} \backslash\left\{B_{1}\right\} \quad \mathcal{S}^{k+1}(B, \cdot)=\mathcal{S}^{k}(B, \cdot) .
\end{aligned}
$$

- If $\mathcal{I}\left(B_{2}^{\prime}, \mathcal{S}^{k}\left(B_{2}, \cdot\right)\right) \leqslant \mathcal{I}\left(B_{2}, \mathcal{S}^{k}\left(B_{2}, \cdot\right)\right)$, then we set

$$
\begin{aligned}
\mathcal{B}^{k+1}= & \left\{B_{2}^{\prime}\right\} \cup \mathcal{B}^{k} \backslash\left\{B_{2}\right\}, \quad \mathcal{S}^{k+1}\left(B_{2}^{\prime}, \cdot\right)=\mathcal{S}^{k}\left(B_{2}, \cdot\right) \\
& \forall B \in \mathcal{B}^{k} \backslash\left\{B_{2}\right\} \quad \mathcal{S}^{k+1}(B, \cdot)=\mathcal{S}^{k}(B, \cdot) .
\end{aligned}
$$

The collection with touching status $\left(\mathcal{B}^{k+1}, S^{k+1}\right)$ satisfies

$$
\begin{gathered}
\mathcal{I}\left(\mathcal{B}^{k+1}, \mathcal{S}^{k+1}\right) \leqslant \mathcal{I}\left(\mathcal{B}^{k}, \mathcal{S}^{k}\right), \quad \bigcup_{B \in \mathcal{B}^{k+1}} B=\bigcup_{B \in \mathcal{B}^{k}} B, \\
\bigcup_{B_{1}, B_{2} \in \mathcal{B}^{k+1}, B_{1} \neq B_{2}} B_{1} \cap B_{2} \subseteq \bigcup_{B_{1}, B_{2} \in \mathcal{B}^{k}, B_{1} \neq B_{2}} B_{1} \cap B_{2},
\end{gathered}
$$

$$
\begin{aligned}
\operatorname{Dist}\left(\left(\mathcal{B}^{k+1}, \mathcal{S}^{k+1}\right),(\mathcal{A}, \mathcal{T})\right) & \leqslant \operatorname{Dist}\left(\left(\mathcal{B}^{k+1}, \mathcal{S}^{k+1}\right),\left(\mathcal{B}^{k}, \mathcal{S}^{k}\right)\right)+\operatorname{Dist}\left(\left(\mathcal{B}^{k}, \mathcal{S}^{k}\right),(\mathcal{A}, \mathcal{T})\right) \\
& \leqslant \mathcal{L}^{d}\left(\bigcup_{B_{1}, B_{2} \in \mathcal{B}^{k}, B_{1} \neq B_{2}} B_{1} \cap B_{2}\right)+\operatorname{Dist}\left(\left(\mathcal{B}^{k}, \mathcal{S}^{k}\right),(\mathcal{A}, \mathcal{T})\right) .
\end{aligned}
$$

Necessarily the algorithm stops at some step $k$ less than $(|\mathcal{A}|-1)|\mathcal{A}|$. The final collection with touching status $(\mathcal{B}, \mathcal{S})$ obtained at the end of the algorithm satisfies the conditions stated in the lemma.

\section{Proofs of the main results}

We prove first the large deviations principle of Theorem 1.8 for FK percolation. The upper bound is split in two parts: exponential tightness (Section 4.1) and a local upper bound (Section 4.2). We then prove the lower bound (Section 4.3). Next comes the proof of Theorem 1.1 in Section 4.4. Finally, the proof of the large deviations principle of Theorem 1.3 for the Potts model is split into three parts: the study of the asymptotics of the conditioning event in Section 4.5, then the lower bound (Section 4.6) and the upper bound (Section 4.7).

\subsection{The exponential tightness estimate for FK percolation}

Let $k$ be a fixed integer. We work with the $(K=k, \alpha=2)$ renormalization process with block variables $X(\underline{x})$ given by the indicator functions of the event $O\left(B^{\prime}(\underline{x}), k-\right.$ $1)^{c}$ for $\underline{x} \in \underline{\Omega}_{n}$. Let $\underline{A}$ be a $\mathbb{L}^{d, \infty}$-connected subset of $\underline{\Omega}_{n}$. We recall that a residual $\mathbb{L}^{d, \infty}$-component $\underline{R}$ of $\underline{A}$ in $\underline{\Omega}_{n}$ is an $\mathbb{L}^{d, \infty}$-connected component of $\underline{\Omega}_{n} \backslash \underline{A}$. We define

$$
\begin{aligned}
& \text { fill } \underline{A}=\underline{A} \cup\left\{\underline{R} ; \underline{R} \text { is a residual } \mathbb{L}^{d, \infty} \text {-component of } \underline{A}\right. \text {, } \\
& \left.\operatorname{diam} \underline{R} \leqslant f(n) / k, \underline{R} \cap \underline{\Omega}_{n}^{\prime} \neq \emptyset\right\}
\end{aligned}
$$

where $\underline{\Omega}_{n}^{\prime}=\left\{\underline{x} \in \underline{\Omega}_{n} ; B(\underline{x}) \cap \Omega_{n}^{\prime} \neq \emptyset\right\}$ (see Section 2 for the notation). Remark that a $\mathbb{L}^{d, \infty}$-connected set $\underline{R}$ such that diam $\underline{R} \leqslant f(n) / k, \underline{R} \cap \underline{\Omega}_{n}^{\prime} \neq \emptyset$ does not intersect $\partial^{i n} \underline{\Omega}_{n}$. 
For a cluster $C$ of the configuration, we set $\underline{C}=\left\{\underline{x} \in \underline{\Omega}_{n} ; B(\underline{x}) \cap C \neq \emptyset\right\}$. Notice that if $C$ is a large cluster, i.e., $C \in \mathcal{C}_{n}$, then $\operatorname{diam} \underline{C}>f(n) / k$. Let $\underline{A}$ be a $\mathbb{L}^{d, \infty}$-connected component of good blocks, i.e., of the set $\left\{\underline{x} \in \underline{\Omega}_{n} ; X(\underline{x})=0\right\}$. Note that there is at most one large cluster intersecting $\underline{A}$ (if there were two then they would be connected via $\underline{A}$ ). Let $\underline{R}$ be a residual $\mathbb{L}^{d, \infty}$-component of $\underline{A}$ such that $\operatorname{diam} \underline{R} \leqslant f(n) / k$ and let $C$ be a large cluster such that $\underline{C} \cap \underline{R} \neq \emptyset$. Since $\underline{C}$ cannot fit into $\underline{R}$ (for reasons of diameter), we have $\underline{C} \cap \partial_{\infty}^{\text {out }} \underline{R} \neq \emptyset$; however $\partial_{\infty}^{\text {out }} \underline{R} \subseteq \underline{A}$ (recall that $\partial_{\infty}^{\text {out }} \underline{R}$ is a connected set of good blocks) so that $\underline{C} \cap \underline{A} \neq \emptyset$. Therefore, if $C$ is a large cluster then either $\underline{C} \cap \underline{A} \neq \emptyset$ or $\underline{C} \cap$ fill $\underline{A}=\emptyset$. For $C \in \mathcal{C}_{n}$ we define

$$
\underline{\widehat{C}}=\bigcup \text { fill } \underline{A}
$$

where the union runs over all the $\mathbb{L}^{d, \infty}$-connected components $\underline{A}$ of good blocks such that $\underline{C} \cap \underline{A} \neq \emptyset$. The previous discussion shows that the sets $\underline{\widehat{C}}, C \in \mathcal{C}_{n}$, are pairwise disjoint. By definition, the $\mathbb{L}^{d, \infty}$ outer boundary of $\underline{\underline{C}}$ consists of bad blocks whenever $\underline{\widehat{C}} \neq \emptyset$. In case $\underline{\widehat{C}}=\emptyset$, we define $\partial_{\infty}^{\text {out }} \widehat{\widehat{C}}$ as $\underline{C}$ which again consists only of bad blocks.

Let now $C$ belong to $\mathcal{C}_{n}$ and let $\underline{F}$ be an $\mathbb{L}^{d, \infty}$-connected component of bad blocks intersecting simultaneously $\partial_{\infty}^{\text {out }} \widehat{\widehat{C}}$ and $\underline{\Omega}_{n}^{\prime}$. We claim that $|\underline{F}|>f(n) / k$. To show this we consider only the case where $\underline{\widehat{C}} \neq \emptyset$ (the case $\underline{\widehat{C}}=\emptyset$ is straightforward) and we assume $|\underline{F}| \leqslant f(n) / k$. The definition of $\underline{\Omega}_{n}$ implies that for $n$ large enough $d_{2}\left(\partial^{i n} \underline{\Omega}_{n}, \underline{\Omega}_{n}^{\prime}\right)>$ $f(n) / k$ therefore $\underline{F} \cap \partial^{i n} \underline{\Omega}_{n}=\emptyset$. Let $\underline{D}=\partial_{\infty}^{\text {out,ext }} \underline{F}$. Then $\underline{D}$ is a $\mathbb{L}^{d, \infty}$ connected set of good blocks surrounding $\underline{F}$, so that $\underline{F}$ is included in a $\mathbb{L}^{d, \infty}$ residual component $\underline{R}$ of $\underline{D}$ satisfying $\operatorname{diam} \underline{R} \leqslant f(n) / k, \underline{R} \cap \underline{\Omega}_{n}^{\prime} \neq \emptyset$. Let $\underline{A}$ be the $\mathbb{L}^{d, \infty}$-connected component of good blocks containing $\underline{D}$. The above properties imply that $\underline{R} \subseteq$ fill $\underline{A}$. Moreover $\partial_{\infty}^{\text {out }} \underline{F} \cap \underline{\widehat{C}} \neq \emptyset$; since diam $\underline{\widehat{C}}>f(n) / k$, necessarily $\left(\partial_{\infty}^{\text {out, ext }} \underline{\underline{F}}\right) \cap \underline{\widehat{C}} \neq \emptyset$, hence $\underline{A} \cap \underline{\widehat{C}} \neq \emptyset$. Either $\underline{A} \cap \underline{C} \neq \emptyset$, whence $\underline{R} \subseteq \underline{\widehat{C}}$, a contradiction. Or there exists a $\mathbb{L}^{d, \infty}$. connected component $\underline{A}^{\prime}$ of good blocks intersecting $\underline{C}$ such that $\underline{A} \cap$ fill $\underline{A}^{\prime} \neq \emptyset$, which implies that $\underline{A} \subset$ fill $\underline{A}^{\prime}$ (any $\mathbb{L}^{d, \infty}$-connected component of good blocks intersecting a residual component of $\underline{A}^{\prime}$ is fully included in this residual component, otherwise it would intersect $\underline{A}^{\prime}$ ) and again $\underline{R} \subseteq$ fill $\underline{A^{\prime}} \subseteq \underline{\widehat{C}}$, which is absurd. Thus $|\underline{F}|>f(n) / k$, as claimed.

Let $\widehat{\underline{E}}$ be the union of all the $\mathbb{L}^{d, \infty}$-connected components of bad blocks intersecting simultaneously $\underline{\Omega}_{n}^{\prime}$ and $\bigcup_{C \in \mathcal{C}_{n}} \partial_{\infty}^{\text {out }} \widehat{\underline{C}}$.

LEMMA 4.1. - There exists $k_{0}=k_{0}(d, \Omega, p, q)$ such that, for $k \geqslant k_{0}$, there exist positive constants $b=b(k, d, \Omega, p, q), c=c(k, d, \Omega, p, q)$ such that for all $s>0$ and $n \in \mathbb{N}$,

$$
\Phi_{n}[|\widehat{\underline{F}}| \geqslant s] \leqslant b \exp (-c s) .
$$

Proof. - We proceed as in Lemma 2.3 in [15] or Lemma 7.9 in [14]. The set $\underline{\hat{F}}$ consists of bad blocks and each of its components has size larger than $f(n) / k$. We first prove the following estimate: for any subset $\underline{A} \subseteq \underline{\Omega}_{n}$,

$$
\Phi_{n}[\underline{\forall} \in \underline{A} ; X(\underline{x})=1] \leqslant \exp \left(4^{-d}|\underline{A}| \log \varepsilon\right),
$$

where $\varepsilon=b \exp (-c k)$ is given by (14). We use the equivalence relation on $\underline{\Omega}_{n}: \underline{x} \approx \underline{y}$ iff 3 divides each component of $\underline{x}-\underline{y}$. Since there are at most $4^{d}$ distinct classes in 
$\underline{\Omega}_{n}$, certainly there exists $\underline{x}^{*} \in \underline{\Omega}_{n}$ such that the intersection of $\underline{A}$ and the equivalence class of $\underline{x}^{*}$ has cardinality at least $4^{-d}|\underline{A}|$. By (18), the field $X(\underline{x}), \underline{x} \in \underline{\Omega}_{n}, \underline{x} \approx \underline{x}^{*}$ is stochastically dominated by a Bernoulli product field with parameter $\varepsilon$. Finally,

$$
\begin{aligned}
\Phi_{n}[\forall \underline{x} \in \underline{A}, X(\underline{x})=1] & \leqslant \Phi_{n}\left[\forall \underline{x} \in\left\{\underline{y} \in \underline{A} ; \underline{y} \approx \underline{x}^{*}\right\} X(\underline{x})=1\right] \\
& \leqslant \exp \left(4^{-d}|\underline{A}| \log \varepsilon\right)
\end{aligned}
$$

as claimed. Now we turn to the statement of the lemma. By decomposing the event in question, we can estimate $\Phi_{n}[|\underline{\underline{F}}| \geqslant s]$ by

$$
\sum_{j \geqslant s} \sum_{1 \leqslant i \leqslant j k / f(n)} \sum_{\substack{m_{1}, m_{2}, \ldots, m_{i} \geqslant f(n) / k \\ m_{1}+m_{2}+\cdots+m_{i}=j}} \sum_{\underline{A}_{1}, \underline{A}_{2}, \ldots, \underline{A}_{i}} \Phi_{n}\left[\forall \underline{x} \in \underline{A}_{1} \cup \underline{A}_{2} \cup \cdots \cup \underline{A}_{i} X(\underline{x})=1\right] .
$$

The ultimate summation extends over the pairwise disjoint sets $\underline{A}_{1}, \ldots, \underline{A}_{i}$ intersecting $\underline{\Omega}_{n}^{\prime}$ such that $\underline{A}_{l}$ is $\mathbb{L}^{d, \infty}$-connected and $\left|\underline{A}_{l}\right|=m_{l}$ for $1 \leqslant l \leqslant i$. By (21), the probability appearing in the summation is less than $\exp \left(4^{-d} j \log \varepsilon\right)$. For fixed $j$ and $i$, there are at most $2^{j}$ ways to choose the values $m_{1}, \ldots, m_{i}$. Recall that there exists a constant $b=b(d)>0$ such that the number of $\mathbb{L}^{d, \infty}$-connected sets of size $m$ containing the origin is bounded by $(b / 2)^{m}$. The number of possibilities for choosing the set $\underline{A}_{l}$ is bounded by $\mathcal{L}^{d}(\Omega)(n / k)^{d} b^{m_{l}}$ for $1 \leqslant l \leqslant i$. Thus the number of terms involved in the last three summations is less than $2 \exp \left((2 j k / f(n)) \log \left(\mathcal{L}^{d}(\Omega)(n / k)^{d}\right)+j \log b\right)$. Putting these estimates together, we get the claim of the lemma.

Our next goal is to define a (random) t-partition associated with the objects $\underline{\widehat{C}}, C \in \mathcal{C}_{n}$. Recall that for a set of (indices of) blocks $\underline{A}, B(\underline{A})$ denotes the union of the blocks indexed by $\underline{A}$. First we set

$$
\underline{\widehat{R}}=\underline{\Omega}_{n} \backslash\left(\underline{\widehat{F}} \cup \bigcup_{C \in \mathcal{C}_{n}} \underline{\widehat{C}}\right)
$$

and for $C^{\mathrm{FK}} \in \mathcal{C}_{n}^{\mathrm{FK}}$,

$$
\widehat{\widehat{C}}^{\mathrm{FK}}=\bigcup_{C \in \mathcal{C}_{n}, C \subseteq C^{\mathrm{FK}}} \underline{\widehat{C}} .
$$

Let $\left(\widehat{\mathcal{A}}_{n}, \widehat{\mathcal{T}}_{n}\right)$ be the element of $\operatorname{TP}(\Omega)$ defined by

$$
\widehat{\mathcal{A}}_{n}=\left\{\Omega_{n}^{\prime} \cap B\left(\underline{\widehat{C}}^{\mathrm{FK}}\right) ; C^{\mathrm{FK}} \in \mathcal{C}_{n}^{\mathrm{FK}}\right\} \cup\left\{\Omega_{n}^{\prime} \cap B(\underline{\widehat{F}}),\left(\Omega \backslash \Omega_{n}^{\prime}\right) \cup B(\underline{\widehat{R}})\right\}
$$

and for $m \in \mathbb{N}, C^{\mathrm{FK}} \in \mathcal{C}_{n}^{\mathrm{FK}}$,

$$
\begin{gathered}
\widehat{\mathcal{T}}_{n}(B(\underline{\widehat{F}}), m)=\widehat{\mathcal{T}}_{n}(B(\underline{\widehat{R}}), m)=0, \\
\widehat{\mathcal{T}}_{n}\left(B\left(\underline{\widehat{C}}^{\mathrm{FK}}\right), m\right)= \begin{cases}1 ; & C^{\mathrm{FK}} \cap \Gamma_{n}^{m} \neq \emptyset, \\
0 ; & \text { otherwise. }\end{cases}
\end{gathered}
$$

We next derive a probabilistic estimate on the surface energy of this t-partition.

LEMmA 4.2. - There exists $k_{0}=k_{0}(d, \Omega, p, q)$ such that, for $k \geqslant k_{0}$, there exist positive constants $c=c(k, d, \Omega, p, q)$ and $\lambda_{0}=\lambda_{0}(d, \Omega, p, q)$ such that for all $\lambda>\lambda_{0}$,

$$
\limsup _{n \rightarrow \infty} \frac{1}{n^{d-1}} \log \Phi_{n}\left[\mathcal{I}\left(\widehat{\mathcal{A}}_{n}, \widehat{\mathcal{T}}_{n}\right) \geqslant \lambda\right] \leqslant-c \lambda .
$$


Proof. - By construction, the interfaces of $\widehat{\mathcal{A}}_{n}$ (that is, the set $\left.\partial^{*}\left(\widehat{\mathcal{A}}_{n}, \widehat{\mathcal{T}}_{n}\right)\right)$ are located either on $\partial \Omega_{n}^{\prime} \cup \Gamma$ or on the faces of the blocks of $\underline{\widehat{F}}$. Thus

$$
\mathcal{I}\left(\widehat{\mathcal{A}}_{n}, \widehat{\mathcal{T}}_{n}\right) \leqslant \tau_{\max }\left(\mathcal{H}^{d-1}(\Gamma)+\mathcal{H}^{d-1}\left(\partial \Omega_{n}^{\prime}\right)+(k / n)^{d-1} 2 d|\underline{\widehat{F}}|\right) .
$$

The desired claim follows from Lemma 4.1.

The next step is to show that the sequences of phase partitions $\left(\mathcal{A}_{n}, \mathcal{T}_{n}\right)_{n \in \mathbb{N}}$ and $\left(\widehat{\mathcal{A}}_{n}, \widehat{\mathcal{T}}_{n}\right)_{n \in \mathbb{N}}$ are exponentially contiguous.

LEMmA 4.3. - There exists $k_{0}=k_{0}(d, \Omega, p, q)$ such that, for $k \geqslant k_{0}$, for each $\delta>0$,

$$
\limsup _{n \rightarrow \infty} \frac{1}{n^{d-1}} \log \Phi_{n}\left[\operatorname{Dist}\left(\left(\mathcal{A}_{n}, \mathcal{T}_{n}\right),\left(\widehat{\mathcal{A}}_{n}, \widehat{\mathcal{T}}_{n}\right)\right) \geqslant \delta\right]=-\infty .
$$

Proof. - We compute first the volume of the $f(n) / n$-neighborhood of $B(\underline{\widehat{F}})$. Let $\underline{F}_{i}$, $i \in I$, be the $\mathbb{L}^{d, \infty}$-connected components of $\widehat{\underline{F}}$. Applying Lemma 2.1,

$$
\begin{aligned}
\mathcal{L}^{d}\left(\mathcal{V}_{\infty}(B(\widehat{\underline{F}}), f(n) / n)\right) & \leqslant \sum_{i \in I} n^{-d} \mathcal{L}^{d}\left(\mathcal{V}_{\infty}\left(n B\left(\underline{F}_{i}\right), f(n)\right)\right) \\
& \leqslant \sum_{i \in I} n^{-d} 4^{d+1} f(n)^{d-1} k^{d}\left|\underline{F}_{i}\right|=4^{d+1}(k / n)^{d} f(n)^{d-1}|\widehat{\widehat{F}}| .
\end{aligned}
$$

By construction, for any $C \in \mathcal{C}_{n}$, we have $\underline{C} \subseteq \underline{\widehat{C}} \cup \underline{\widehat{F}}$. By setting

$$
E=\left\{x \in \Omega ; \forall C \in \mathcal{C}_{n}, \mathrm{~d}_{\infty}(x, C) \geqslant f(n) / n\right\}
$$

we have

$$
\Omega_{n}^{\prime} \cap B(\underline{\widehat{R}}) \backslash \mathcal{V}_{\infty}(B(\underline{\widehat{F}}), f(n) / n) \subseteq \Omega_{n}^{\prime} \cap E .
$$

(Indeed, any $\mathbb{L}^{d, \infty}$-component of good blocks in $\underline{\Omega}_{n}^{\prime}$ is surrounded by a $\mathbb{L}^{d, \infty}$-connected set of bad blocks which is included in $\underline{\underline{F}}$.)

In order to estimate the volume of $E$ we work with the $(K=f(n), \alpha=2)$ renormalization process. Note that $\underline{\Omega}_{n}$ is different from the previous one since the block size is now different. The block variable $Y(\underline{x})$ is the indicator function of the event $O\left(B^{\prime}(\underline{x}), f(n)\right)^{c}$. If $Y(\underline{x})=0$ then there exists a unique large cluster $C$ intersecting $B(\underline{x})$ and $B(\underline{x}) \cap E=\emptyset$. Therefore,

$$
\mathcal{L}^{d}\left(E \cap \Omega_{n}^{\prime}\right) \leqslant(f(n) / n)^{d} \sum_{\underline{x}_{\in} \underline{\Omega}_{n}} Y(\underline{x}) .
$$

We derive an upper bound on the distance between $\left(\mathcal{A}_{n}, \mathcal{T}_{n}\right)$ and $\left(\widehat{\mathcal{A}}_{n}, \widehat{\mathcal{T}}_{n}\right)$ by considering an arrangement between the two t-partitions in which $B\left(\underline{\widehat{C}}^{\mathrm{FK}}\right)$ corresponds to vor $C^{\mathrm{FK}}$, for every $C^{\mathrm{FK}} \in \mathcal{C}_{n}^{\mathrm{FK}}$ :

$$
\begin{aligned}
\operatorname{Dist}\left(\left(\mathcal{A}_{n}, \mathcal{T}_{n}\right),\left(\widehat{\mathcal{A}}_{n}, \widehat{\mathcal{T}}_{n}\right)\right) \leqslant & 2 \mathcal{L}^{d}\left(\Omega \backslash \Omega_{n}^{\prime}\right)+\mathcal{L}^{d}\left(\Omega_{n}^{\prime} \cap B(\underline{\widehat{F}})\right)+\mathcal{L}^{d}\left(\Omega_{n}^{\prime} \cap B(\underline{\widehat{R}})\right) \\
& +\sum_{C^{\mathrm{FK}} \in \mathcal{C}_{n}^{\mathrm{FK}}} \operatorname{dist}_{L^{1}}\left(\Omega_{n}^{\prime} \cap B\left(\underline{\widehat{C}}^{\mathrm{FK}}\right), \Omega_{n}^{\prime} \cap \operatorname{vor} C^{\mathrm{FK}}\right)
\end{aligned}
$$




$$
\begin{aligned}
\leqslant & 2 \mathcal{L}^{d}\left(\Omega \backslash \Omega_{n}^{\prime}\right)+\mathcal{L}^{d}\left(\Omega_{n}^{\prime} \cap B(\underline{\widehat{F}})\right)+\mathcal{L}^{d}\left(\Omega_{n}^{\prime} \cap B(\underline{\widehat{R}})\right) \\
& +\sum_{C \in \mathcal{C}_{n}} \operatorname{dist}_{L^{1}}\left(\Omega_{n}^{\prime} \cap B(\underline{\widehat{C}}), \Omega_{n}^{\prime} \cap \operatorname{vor} C\right) .
\end{aligned}
$$

Yet, $\Omega_{n}^{\prime} \cap B(\underline{\widehat{C}}) \subseteq \Omega_{n}^{\prime} \cap$ vor $C$. Since $\widehat{\mathcal{A}}_{n}$ is a partition and the sets vor $C, C \in \mathcal{C}_{n}$, are pairwise disjoint, we have

$$
\begin{aligned}
\sum_{C \in \mathcal{C}_{n}} \operatorname{dist}_{L^{1}}\left(\Omega_{n}^{\prime} \cap B(\underline{\widehat{C}}), \Omega_{n}^{\prime} \cap \operatorname{vor} C\right) & =\sum_{C \in \mathcal{C}_{n}} \mathcal{L}^{d}\left(\Omega_{n}^{\prime} \cap \operatorname{vor} C \backslash B(\underline{\widehat{C}})\right) \\
& \leqslant \mathcal{L}^{d}\left(\Omega_{n}^{\prime} \backslash \bigcup_{C \in \mathcal{C}_{n}} B(\underline{\widehat{C}})\right) \\
& \leqslant \mathcal{L}^{d}\left(\Omega_{n}^{\prime} \cap B(\underline{\widehat{F}})\right)+\mathcal{L}^{d}\left(\Omega_{n}^{\prime} \cap B(\underline{\widehat{R}})\right) .
\end{aligned}
$$

Finally,

$$
\begin{aligned}
& \operatorname{Dist}\left(\left(\mathcal{A}_{n}, \mathcal{T}_{n}\right),\left(\widehat{\mathcal{A}}_{n}, \widehat{\mathcal{T}}_{n}\right)\right) \\
& \quad \leqslant 2 \mathcal{L}^{d}\left(\Omega \backslash \Omega_{n}^{\prime}\right)+2 \mathcal{L}^{d}\left(\Omega_{n}^{\prime} \cap B(\underline{\widehat{F}})\right)+2 \mathcal{L}^{d}\left(\Omega_{n}^{\prime} \cap B(\underline{\widehat{R}})\right) \\
& \quad \leqslant 2 \mathcal{L}^{d}\left(\Omega \backslash \Omega_{n}^{\prime}\right)+2 \mathcal{L}^{d}\left(\Omega_{n}^{\prime} \cap B(\underline{\widehat{F}})\right)+2 \mathcal{L}^{d}\left(\Omega_{n}^{\prime} \cap E\right)+2 \mathcal{L}^{d}\left(\mathcal{V}_{\infty}(B(\underline{\widehat{F}}), f(n) / n)\right) \\
& \quad \leqslant 2 \mathcal{L}^{d}\left(\Omega \backslash \Omega_{n}^{\prime}\right)+4^{d+2}(k / n)^{d} f(n)^{d-1}|\underline{\widehat{F}}|+2(f(n) / n)^{d} \sum_{\underline{x} \in \underline{\Omega}_{n}} Y(\underline{x}) .
\end{aligned}
$$

The estimate obtained in Lemma 4.1 yields

$$
\forall \delta>0, \quad \limsup _{n \rightarrow \infty} \frac{1}{n^{d-1}} \log \Phi_{n}\left[|\underline{\widehat{F}}| \geqslant \delta n^{d} / f(n)^{d-1}\right]=-\infty .
$$

By the estimate (14) and Lemma 2.3, we have also

$$
\forall \delta>0, \quad \limsup _{n \rightarrow \infty} \frac{1}{n^{d-1}} \log \Phi_{n}\left[2(f(n) / n)^{d} \sum_{\underline{x} \in \underline{\Omega}_{n}} Y(\underline{x})>\delta\right]=-\infty
$$

and the exponential contiguity (23) follows directly from these estimates.

An immediate consequence of Lemmas 4.2 and 4.3 is the exponential tightness of the sequence of t-partitions $\left(\mathcal{A}_{n}, \mathcal{T}_{n}\right)_{n \in \mathbb{N}}$ : there exists $c=c(d, \Omega, p, q)>0$ and $\lambda_{0}>0$ such that for every $\lambda>\lambda_{0}$, every $\delta>0$,

$$
\limsup _{n \rightarrow \infty} \frac{1}{n^{d-1}} \log \Phi_{n}\left[\operatorname{Dist}\left(\left(\mathcal{A}_{n}, \mathcal{T}_{n}\right), \mathcal{I}^{-1}([0, \lambda])\right)>\delta\right] \leqslant-c \lambda .
$$

\subsection{Local upper bound estimate for Theorem 1.8 (FK percolation)}

We start by recalling some essential results from [15]. Let $\Theta \subseteq \mathbb{R}^{d}$ be a box building, i.e., the union of finitely many $d$-dimensional boxes with nonempty interior. Fix a monotone increasing function $\phi: \mathbb{N} \rightarrow \mathbb{N}$ satisfying $\lim _{n \rightarrow \infty} \phi(n)=\infty$ and $\lim _{n \rightarrow \infty} \phi(n) / n=0$. We will consider the $\phi(n) / n$-interior of the building $\Theta$ which is defined as

$$
\operatorname{int}(\Theta, \phi(n) / n)=\left\{x \in \Theta ; \mathrm{d}_{\infty}\left(x, \partial^{i n} \Theta\right)>\phi(n) / n\right\} .
$$


Proposition 4.4. - Assume $d \geqslant 3, q \geqslant 1, p>\widehat{p}_{c}$ with $\theta^{f}(p)=\theta^{w}(p)$. Let $S_{n}$ be a sequence of events such that $S_{n}$ depends only on the edges in $\operatorname{int}(\Theta, \phi(n) / n)$ and for each $n \in \mathbb{N}$, let $\pi(n)$ be a partially wired b.c. on $\Theta$. Then

$$
\limsup _{n \rightarrow \infty} \frac{1}{n^{d-1}} \log \Phi_{\Theta}^{\pi(n), p, q}\left[S_{n}\right]=\limsup _{n \rightarrow \infty} \frac{1}{n^{d-1}} \log \Phi_{\Theta}^{f, p, q}\left[S_{n}\right] .
$$

The same equality is valid when lim sup is replaced by lim inf.

LEMMA 4.5 (Decoupling lemma). - Let $d \geqslant 3, q \geqslant 1, p>\widehat{p}_{c}$ with $\theta^{f}(p)=\theta^{w}(p)$. Let $D_{i}, i \in I$, be a finite collection of disjoint compact subsets of $\Omega \cup \Gamma$. Assume that these sets have non-empty connected interiors. For $i \in I$ let $S_{n}^{i}$ be a sequence of events such that $S_{n}^{i}$ depends only on the edges in $D_{i} \cap \Omega_{n}$. Then

$$
\limsup _{n \rightarrow \infty} \frac{1}{n^{d-1}} \log \Phi_{n}\left[\bigcap_{i \in I} S_{n}^{i}\right] \leqslant \sum_{i \in I} \limsup _{n \rightarrow \infty} \frac{1}{n^{d-1}} \log \Phi_{n}\left[S_{n}^{i}\right] .
$$

Notation. - Let $B(x, r)$ be a ball in $\Omega$, let $w$ belong to $S^{d-1}, n$ to $\mathbb{N}$ and let $r, \delta$ be positive. Recall that the open $B(x, r)$-clusters are the open clusters in the configuration restricted to the ball $B(x, r)$. Let $\operatorname{Sep}(n, x, r, w, \delta)$ be the event: there exists a collection $\mathcal{C}$ of open $B(x, r)$-clusters such that

$$
\operatorname{dist}_{L^{1}}\left(\bigcup_{C \in \mathcal{C}} \bigcup_{x \in C} \Lambda(x, 1 / n), B_{-}(x, r, w)\right) \leqslant \delta r^{d} .
$$

Let next $B(x, r)$ be a ball such that $x$ belongs to $\partial^{*} \Omega$. Let $n \in \mathbb{N}$ and $\delta>0$. Let $\operatorname{Sep}^{\mathrm{bd}}(n, x, r, \delta)$ be the event: there exists a collection $\mathcal{C}$ of open $B_{-}\left(x, r, v_{\Gamma}(x)\right)$-clusters such that none of them intersects $\partial^{i n} \Omega_{n}$ and the inequality (25) is satisfied with $w$ replaced by $v_{\Gamma}(x)$.

LEMMA 4.6 (Interface lemma). - Let $d \geqslant 3, q \geqslant 1, p>\widehat{p}_{c}, \theta^{f}(p)=\theta^{w}(p)$. There exists a constant $c=c(p, q, d)$ such that for every $r$ in $(0,1)$, every ball $B(x, r) \subseteq \Omega$, every unit vector $w$ in $S^{d-1}$, and every $\delta$ in $(0, \theta / 2)$,

$$
\limsup _{n \rightarrow \infty} \frac{1}{n^{d-1}} \log \Phi_{n}[\operatorname{Sep}(n, x, r, w, \delta)] \leqslant-\alpha_{d-1} r^{d-1} \tau(w)\left(1-c \delta^{1 / 2}\right)
$$

and for $m \in \mathbb{N}$, for every ball $B(x, r)$ such that $x \in \Gamma^{m} \cap \partial^{*} \Omega$ and $B(x, r) \cap \Gamma \subseteq \Gamma^{m}$,

$$
\limsup _{n \rightarrow \infty} \frac{1}{n^{d-1}} \log \Phi_{n}\left[\operatorname{Sep}^{\mathrm{bd}}(n, x, r, \delta)\right] \leqslant-\alpha_{d-1} r^{d-1} \tau\left(v_{\Gamma}(x)\right)\left(1-c \delta^{1 / 2}\right) .
$$

The first estimate was proved in [15]. We explain briefly how to derive the second estimate. Let $x \in \Gamma^{m} \cap \partial^{*} \Omega$ for some $m \in \mathbb{N}$ and let $r$ be such that $B(x, r) \cap \Gamma \subseteq \Gamma^{m}$. We remark first that the event $\operatorname{Sep}^{\mathrm{bd}}(n, x, r, \delta)$ is decreasing. Let $\Theta$ be a box building containing $B(x, r)$ in its interior and such that $\Theta \cap \Gamma \subseteq \Gamma^{m}$ (such a box building exists because $\Gamma^{m}$ is a relatively open subset of $\Gamma$ ). Let $\Phi_{n}^{\prime}$ be the FK measure in $\Theta \cap \Omega$ induced by the following b.c.:

- the points of $\Theta \cap \Gamma^{m}$ are wired together;

- the points of $\partial(\Theta \cap \Omega) \backslash \Gamma^{m}$ are let free. 
Let $\Phi_{n}^{\prime \prime}$ be the FK measure in $\Theta$ induced by the following b.c.:

- the points of $\partial \Theta \backslash \Omega$ are wired together;

- the points of $\partial(\Theta \cap \Omega) \backslash \Gamma^{m}$ are let free.

By the monotonicity of FK measures with respect to boundary conditions,

$$
\Phi_{n}\left[\operatorname{Sep}^{\mathrm{bd}}(n, x, r, \delta)\right] \leqslant \Phi_{n}^{\prime}\left[\operatorname{Sep}^{\mathrm{bd}}(n, x, r, \delta)\right] \leqslant \Phi_{n}^{\prime \prime}\left[\operatorname{Sep}\left(n, x, r, v_{\Gamma}(x), \delta\right)\right] .
$$

The second estimate stated in Lemma 4.6 is then obtained by applying successively Proposition 4.4 and the first estimate stated in Lemma 4.6.

Our next goal is to construct a t-partition which is exponentially contiguous to the empirical t-partition $\left(\mathcal{A}_{n}, \mathcal{T}_{n}\right)$ and whose boundary corresponds to closed edges. First we define a partition of $\Omega_{n}$ by attaching each small cluster of the configuration not intersecting $\Gamma_{n}$ to the closest large cluster. To break ties we use an arbitrary deterministic rule. For $C \in \mathcal{C}_{n}$, we denote by $\widetilde{C}$ the corresponding continuous region: $\widetilde{C}$ is the union of all cubes $\Lambda(x, 1 / n)$ centered at sites in either $C$ or some of the small clusters attached to $C$. We define for $C^{\mathrm{FK}} \in \mathcal{C}_{n}^{\mathrm{FK}}$

$$
\widetilde{C}^{\mathrm{FK}}=\bigcup_{C \in \mathcal{C}_{n}, C \subseteq C^{\mathrm{FK}}} \widetilde{C} .
$$

We then define a random t-partition of $\Omega$ by setting

$$
\widetilde{\mathcal{A}}_{n}=\left\{\Omega \cap \widetilde{C}^{\mathrm{FK}} ; C^{\mathrm{FK}} \in \mathcal{C}_{n}^{\mathrm{FK}}\right\} \cup\left\{G_{n}\right\},
$$

where $G_{n}=\Omega \cap \bigcup_{x} \Lambda(x, 1 / n)$ and the union runs over all the vertices of the small clusters intersecting $\Gamma_{n}$. The touching functions are given by

$$
\begin{gathered}
\forall C^{\mathrm{FK}} \in \mathcal{C}_{n}^{\mathrm{FK}} \quad \forall m \in \mathbb{N} \quad \widetilde{\mathcal{T}}_{n}\left(\widetilde{C}^{\mathrm{FK}}, m\right)= \begin{cases}1 ; & C^{\mathrm{FK}} \cap \Gamma_{n}^{m} \neq \emptyset, \\
0 ; & \text { otherwise },\end{cases} \\
\forall m \in \mathbb{N} \quad \widetilde{\mathcal{T}}_{n}\left(G_{n}, m\right)=0 .
\end{gathered}
$$

We first show that $\left(\widetilde{\mathcal{A}}_{n}, \widetilde{\mathcal{T}}_{n}\right)_{n \in \mathbb{N}}$ and $\left(\widehat{\mathcal{A}}_{n}, \widehat{\mathcal{T}}_{n}\right)_{n \in \mathbb{N}}$ are exponentially contiguous.

LEMMA 4.7. - For each $\delta>0$,

$$
\limsup _{n \rightarrow \infty} \frac{1}{n^{d-1}} \log \Phi_{n}\left[\operatorname{Dist}\left(\left(\widetilde{\mathcal{A}}_{n}, \widetilde{\mathcal{T}}_{n}\right),\left(\widehat{\mathcal{A}}_{n}, \widehat{\mathcal{T}}_{n}\right)\right) \geqslant \delta\right]=-\infty .
$$

Proof. - By considering the natural arrangements of $\left(\widehat{\mathcal{A}}_{n}, \widehat{\mathcal{T}}_{n}\right)$ and $\left(\widetilde{\mathcal{A}}_{n}, \widetilde{\mathcal{T}}_{n}\right)$ in which $B\left(\underline{\widehat{C}}^{\mathrm{FK}}\right)$ corresponds to $\widetilde{C}^{\mathrm{FK}}$ for each $C^{\mathrm{FK}} \in \mathcal{C}_{n}^{\mathrm{FK}}$, we obtain immediately

$$
\begin{aligned}
& \operatorname{Dist}\left(\left(\widetilde{\mathcal{A}}_{n}, \widetilde{\mathcal{T}}_{n}\right),\left(\widehat{\mathcal{A}}_{n}, \widehat{\mathcal{T}}_{n}\right)\right) \\
& \leqslant \sum_{C^{\mathrm{FK}} \in \mathcal{C}_{n}^{\mathrm{FK}}} \operatorname{dist}_{L^{1}}\left(\Omega_{n}^{\prime} \cap B\left(\underline{\widehat{C}}^{\mathrm{FK}}\right), \Omega_{n}^{\prime} \cap \widetilde{C}^{\mathrm{FK}}\right)+\mathcal{L}^{d}\left(\Omega_{n}^{\prime} \cap B(\underline{\widehat{F}})\right) \\
& \quad+\mathcal{L}^{d}\left(\Omega_{n}^{\prime} \cap B(\underline{\widehat{R}})\right)+2 \mathcal{L}^{d}\left(\Omega \backslash \Omega_{n}^{\prime}\right)
\end{aligned}
$$




$$
\begin{aligned}
\leqslant & \sum_{C \in \mathcal{C}_{n}} \operatorname{dist}_{L^{1}}\left(\Omega_{n}^{\prime} \cap B(\underline{\widehat{C}}), \Omega_{n}^{\prime} \cap \widetilde{C}\right)+\mathcal{L}^{d}\left(\Omega_{n}^{\prime} \cap B(\underline{\widehat{F}})\right)+\mathcal{L}^{d}\left(\Omega_{n}^{\prime} \cap B(\underline{\widehat{R}})\right) \\
& +2 \mathcal{L}^{d}\left(\Omega \backslash \Omega_{n}^{\prime}\right) .
\end{aligned}
$$

From our constructions it follows that for each large cluster $C$, we have $\Omega_{n}^{\prime} \cap B(\underline{\widehat{C}}) \subseteq$ $\Omega_{n}^{\prime} \cap \widetilde{C}$. Since $\widehat{\mathcal{A}}_{n}$ and $\widetilde{\mathcal{A}}_{n}$ are partitions and the sets $\widetilde{C}, C \in \mathcal{C}_{n}$, are pairwise disjoint, we have

$$
\begin{aligned}
\sum_{C \in \mathcal{C}_{n}} \operatorname{dist}_{L^{1}}\left(\Omega_{n}^{\prime} \cap B(\underline{\widehat{C}}), \Omega_{n}^{\prime} \cap \widetilde{C}\right) & \leqslant \mathcal{L}^{d}\left(\Omega_{n}^{\prime} \backslash \bigcup_{C \in \mathcal{C}_{n}} B(\underline{\widehat{C}})\right) \\
& \leqslant \mathcal{L}^{d}\left(\Omega_{n}^{\prime} \cap B(\underline{\widehat{F}})\right)+\mathcal{L}^{d}\left(\Omega_{n}^{\prime} \cap B(\underline{\widehat{R}})\right),
\end{aligned}
$$

whence

$$
\operatorname{Dist}\left(\left(\widetilde{\mathcal{A}}_{n}, \widetilde{\mathcal{T}}_{n}\right),\left(\widehat{\mathcal{A}}_{n}, \widehat{\mathcal{T}}_{n}\right)\right) \leqslant 2 \mathcal{L}^{d}\left(\Omega \backslash \Omega_{n}^{\prime}\right)+2 \mathcal{L}^{d}\left(\Omega_{n}^{\prime} \cap B(\underline{\widehat{F}})\right)+2 \mathcal{L}^{d}\left(\Omega_{n}^{\prime} \cap B(\underline{\widehat{R}})\right)
$$

and the conclusion follows from the final estimates obtained in the proof of Lemma 4.3.

Finally, we estimate the probability that $\left(\mathcal{A}_{n}, \mathcal{T}_{n}\right)$ is close to a fixed element of $\operatorname{TP}(\Omega)$.

Lemma 4.8. $-\operatorname{For}(\mathcal{A}, \mathcal{T}) \in \operatorname{TP}(\Omega)$ and $\varepsilon>0$, there exists $\delta=\delta((\mathcal{A}, \mathcal{T}), \varepsilon)>0$ such that,

$$
\limsup _{n \rightarrow \infty} \frac{1}{n^{d-1}} \log \Phi_{n}\left[\operatorname{Dist}\left(\left(\mathcal{A}_{n}, \mathcal{T}_{n}\right),(\mathcal{A}, \mathcal{T})\right)<\delta\right] \leqslant-(1-\varepsilon) \mathcal{I}(\mathcal{A}, \mathcal{T}) .
$$

Proof. - By the triangle inequality for Dist and Lemmas 4.3 and 4.7, we need only to prove the above statement for the t-partition $\left(\widetilde{\mathcal{A}}_{n}, \widetilde{\mathcal{T}}_{n}\right)$. Let $(\mathcal{A}, \mathcal{T})$ be an element of $\operatorname{TP}(\Omega)$. For $\varepsilon>0$, set $\varepsilon^{\prime}=\varepsilon(1+1 / \mathcal{I}(\mathcal{A}, \mathcal{T}))^{-1}$. Pick $\delta_{0} \in(0, \theta / 2)$ such that $c \sqrt{\delta_{0}}<\varepsilon^{\prime}$ where $c=c(p, q, d)$ is the constant appearing in the interface Lemma 4.6. Let $B\left(x_{i}, r_{i}\right)$, $i \in I_{0} \cup I_{1}$, be a finite collection of disjoint balls associated with $(\mathcal{A}, \mathcal{T}), \varepsilon^{\prime}$ and $\delta_{0} / 3$, as given in the covering Lemma 3.4. Let $\delta>0$ be such that

$$
\forall i \in I_{0} \cup I_{1} \quad \delta<\delta_{0} r_{i}^{d} / 3 \quad \text { and } \quad \forall i \in I_{1} \quad \delta<2^{-m_{i}} .
$$

Suppose that $\operatorname{Dist}\left(\left(\widetilde{\mathcal{A}}_{n}, \widetilde{\mathcal{T}}_{n}\right),(\mathcal{A}, \mathcal{T})\right)<\delta$. Then for $i \in I_{0}$, there exist $A_{-}^{i} \in \mathcal{A}, A_{n}^{i} \in \widetilde{\mathcal{A}}_{n}$, $v_{i} \in S^{d-1}$ such that

$$
\begin{gathered}
\operatorname{dist}_{L^{1}}\left(A_{-}^{i} \cap B\left(x_{i}, r_{i}\right), B_{-}\left(x_{i}, r_{i}, v_{i}\right)\right) \leqslant \delta_{0} r_{i}^{d} / 3, \\
\operatorname{dist}_{L^{1}}\left(A_{-}^{i}, A_{n}^{i}\right)<\delta,
\end{gathered}
$$

and for $i \in I_{1}$, there exist $A_{-}^{i} \in \mathcal{A}, A_{n}^{i} \in \widetilde{\mathcal{A}}_{n}, v_{i} \in S^{d-1}$ such that

$$
\begin{gathered}
\mathcal{T}\left(A_{-}^{i}, m_{i}\right)=0, \quad B\left(x_{i}, r_{i}\right) \cap \Gamma \subset \Gamma^{m_{i}}, \\
\operatorname{dist}_{L^{1}}\left(A_{-}^{i} \cap B\left(x_{i}, r_{i}\right), B_{-}\left(x_{i}, r_{i}, v_{i}\right)\right) \leqslant \delta_{0} r_{i}^{d} / 3, \\
2^{-m_{i}} \mathcal{T}\left(A_{n}^{i}, m_{i}\right)+\operatorname{dist}_{L^{1}}\left(A_{-}^{i}, A_{n}^{i}\right)<\delta
\end{gathered}
$$


and finally

$$
\left|\mathcal{I}(\mathcal{A}, \mathcal{T})-\sum_{i \in I_{0} \cup I_{1}} \alpha_{d-1} r_{i}^{d-1} \tau\left(v_{i}\right)\right| \leqslant \varepsilon^{\prime} .
$$

The penultimate inequality implies in particular that $\mathcal{T}\left(A_{n}^{i}, m_{i}\right)=0$, hence none of the open clusters included in $A_{n}^{i}$ intersects $\Gamma_{n}^{m_{i}}$. For $i$ in $I_{0} \cup I_{1}$ let $\mathcal{C}(i)$ be the collection of the open clusters of the configuration restricted to $A_{n}^{i} \cap B\left(x_{i}, r_{i}\right)$. Note that for any $i \in I_{0} \cup I_{1}$, the clusters of $\mathcal{C}(i)$ are open clusters of the configuration restricted to $B\left(x_{i}, r_{i}\right)$. We have

$$
\begin{aligned}
& \operatorname{dist}_{L^{1}}\left(\bigcup_{C \in \mathcal{C}(i)} \bigcup_{x \in C} \Lambda(x, 1 / n), B_{-}\left(x_{i}, r_{i}, v_{i}\right)\right) \\
& \leqslant \operatorname{dist}_{L^{1}}\left(\bigcup_{C \in \mathcal{C}(i)} \bigcup_{x \in C} \Lambda(x, 1 / n), A_{n}^{i} \cap B\left(x_{i}, r_{i}\right)\right) \\
& \quad+\operatorname{dist}_{L^{1}}\left(A_{n}^{i} \cap B\left(x_{i}, r_{i}\right), A_{-}^{i} \cap B\left(x_{i}, r_{i}\right)\right)+\operatorname{dist}_{L^{1}}\left(A_{-}^{i} \cap B\left(x_{i}, r_{i}\right), B_{-}\left(x_{i}, r_{i}, v_{i}\right)\right) \\
& \leqslant c^{\prime}(d) / n+\delta+\delta_{0} r_{i}^{d} / 3 \leqslant \delta_{0} r_{i}^{d},
\end{aligned}
$$

where $c^{\prime}(d)$ is an appropriate constant depending only on the dimension. If $i$ belongs to $I_{0}$ then the collection $\mathcal{C}(i)$ realizes the event $\operatorname{Sep}\left(n, x_{i}, r_{i}, v_{i}, \delta_{0}\right)$. If $i$ belongs to $I_{1}$ then the collection $\mathcal{C}(i)$ realizes the event $\operatorname{Sep}^{\text {bd }}\left(n, x_{i}, r_{i}, \delta_{0}\right)$. We conclude that

$$
\left\{\operatorname{Dist}\left(\left(\widetilde{\mathcal{A}}_{n}, \widetilde{\mathcal{T}}_{n}\right),(\mathcal{A}, \mathcal{T})\right)<\delta\right\} \subseteq \bigcap_{i \in I_{0}} \operatorname{Sep}\left(n, x_{i}, r_{i}, v_{i}, \delta_{0}\right) \cap \bigcap_{i \in I_{1}} \operatorname{Sep}^{\mathrm{bd}}\left(n, x_{i}, r_{i}, \delta_{0}\right) .
$$

Note that the sets $B\left(x_{i}, r_{i}\right), i \in I_{0} \cup I_{1}$, are compact and disjoint. The decoupling Lemma 4.5 and the interface Lemma 4.6 together imply

$$
\begin{aligned}
& \limsup _{n \rightarrow \infty} \frac{1}{n^{d-1}} \log \Phi_{n}\left[\operatorname{Dist}\left(\left(\widetilde{\mathcal{A}}_{n}, \widetilde{\mathcal{T}}_{n}\right),(\mathcal{A}, \mathcal{T})\right)<\delta\right] \\
& \quad \leqslant-\sum_{i \in I_{0} \cup I_{1}} \alpha_{d-1} r_{i}^{d-1} \tau\left(v_{i}\right)\left(1-c \sqrt{\delta_{0}}\right) \\
& \quad \leqslant-\mathcal{I}(\mathcal{A}, \mathcal{T})\left(1-\varepsilon^{\prime}\right)+\varepsilon^{\prime}=-\mathcal{I}(\mathcal{A}, \mathcal{T})(1-\varepsilon) .
\end{aligned}
$$

The exponential tightness (24) and the local estimate given by Lemma 4.8 yield in a standard way the large deviations upper bound of Theorem 1.8.

\subsection{Proof of the lower bound in Theorem 1.8 (FK percolation)}

We start with two preliminary lemmas. Let $U \subseteq O$ be open connected subsets of $\Omega$ such that $\mathrm{d}_{2}(U, \Omega \backslash O)>0$. We suppose also that there exist a finite number of indices $m_{1}, \ldots, m_{r}$ such that

$$
\begin{gathered}
\forall i \in\{1, \ldots, r\} \quad \partial U \cap \Gamma^{m_{i}} \neq \emptyset, \\
\forall m \in \mathbb{N} \backslash\left\{m_{1}, \ldots, m_{r}\right\} \quad \partial O \cap \Gamma^{m}=\emptyset .
\end{gathered}
$$

We denote by full $(O, U, n)$ the event that 
- for the configuration restricted to $O$ there exists an open cluster $C$ such that $\Omega_{n}^{\prime} \cap U$ is included in $\mathcal{V}_{\infty}(C, f(n) / n)$,

- for $i=1, \ldots, r$, we have $C \cap \Gamma_{m_{i}}^{n} \neq \emptyset$,

- no other large cluster intersects $\mathcal{V}_{\infty}\left(\Omega_{n}^{\prime} \cap U, f(n) / n\right)$.

LEMMA 4.9. - Let $\left(E_{n}\right)_{n \in \mathbb{N}}$ be an arbitrary sequence of events depending on the configuration restricted to $\Omega \backslash O$. Then

$$
\liminf _{n \rightarrow \infty} \frac{1}{n^{d-1}} \log \Phi_{n}\left[\operatorname{full}(O, U, n) \mid E_{n}\right]=0 .
$$

Proof. - Let $\delta>0$. We work with the $(K=\delta n, \alpha=2)$ renormalization process with block variables $X(\underline{x})$ given by the indicator functions of the event $O\left(B^{\prime}(\underline{x}), f(n)\right)^{c}$ for $\underline{x} \in \underline{\Omega}_{n}$. For $\delta$ small and $n$ large enough, there exists a connected set $\underline{A}$ such that $\mathcal{V}_{\infty}\left(\Omega_{n}^{\prime} \cap U, 2 f(n) / n\right) \subseteq B(\underline{A}) \subseteq O$ (we recall that $B(\underline{A})$ is the union of the blocks indexed by $\underline{A}$ ) and moreover $|\underline{A}| \leqslant c$ for a $c=c(\delta, \Omega, U, O)$ independent of $n$. Our hypothesis on $\Omega$ implies that each point of $\partial \Omega$ is accessible from $\Omega$ through a rectifiable path. As a consequence, for $n \in \mathbb{N}$ and for $i=1, \ldots, r$, there exists a path $\gamma_{i}^{n}$ of edges in $\Omega_{n} \backslash B(\underline{A})$ joining a vertex of $\Gamma_{n}^{m_{i}}$ to a vertex belonging to one face $F_{i}^{n}$ of a block belonging to $\underline{A}$ and such that $\left|\gamma_{i}^{n}\right| \leqslant c^{\prime} n$ where $c^{\prime}=c^{\prime}(\delta, \Omega)$ is a constant depending on $\delta$ and $\Omega$ only. Let $T_{i}^{n}$ be the event

$$
T_{i}^{n}=\left\{\text { all the edges in } \gamma_{i}^{n} \cup F_{i}^{n} \text { are open }\right\} .
$$

We have

$$
\bigcap_{\underline{x} \in \underline{A}}\{X(\underline{x})=0\} \cap \bigcap_{1 \leqslant i \leqslant r} T_{i}^{n} \subseteq \text { full }(O, U, n) .
$$

Therefore

$$
\Phi_{n}\left[\operatorname{full}(O, U, n) \mid E_{n}\right] \geqslant \Phi_{n}\left[\bigcap_{\underline{x} \in \underline{A}}\{X(\underline{x})=0\} \mid E_{n} \cap \bigcap_{1 \leqslant i \leqslant r} T_{i}^{n}\right] \Phi_{n}\left[\bigcap_{1 \leqslant i \leqslant r} T_{i}^{n} \mid E_{n}\right] .
$$

Since the events $T_{i}^{n}, i=1, \ldots, r$, do not depend on the edges belonging to the interior of the blocks $B(\underline{A})$, and since the cardinality of $\underline{A}$ is bounded, the uniform estimate (14) implies

$$
\lim _{n \rightarrow \infty} \Phi_{n}\left[\bigcap_{\underline{x} \in \underline{A}}\{X(\underline{x})=0\} \mid E_{n} \cap \bigcap_{1 \leqslant i \leqslant r} T_{i}^{n}\right]=1 .
$$

By the FKG inequality, we have

$$
\begin{aligned}
\Phi_{n}\left[\bigcap_{1 \leqslant i \leqslant r} T_{i}^{n} \mid E_{n}\right] & \geqslant \exp \left(\sum_{1 \leqslant i \leqslant r}\left(\left|\gamma_{i}^{n}\right|+\left|F_{i}^{n}\right|\right) \log \left(\frac{p}{p+q(1-p)}\right)\right) \\
& \geqslant \exp \left(\left(c^{\prime} n r+(\delta n)^{d-1} r\right) \log \left(\frac{p}{p+q(1-p)}\right)\right) .
\end{aligned}
$$


We obtain finally that

$$
\liminf _{n \rightarrow \infty} \frac{1}{n^{d-1}} \log \Phi_{n}\left[\text { full }(O, U, n) \mid E_{n}\right] \geqslant \delta^{d-1} r \log \left(\frac{p}{p+q(1-p)}\right) .
$$

We conclude by sending $\delta$ to 0 .

Let $A$ be an hyperset in $\mathbb{R}^{d}$ and let $r$ be positive or infinite. We denote by $S(A, r)$ the event that there exists a finite set of closed edges in cyl $A \cap \mathcal{V}_{2}($ hyp $A, r$ ) which separates $\infty$ in $\operatorname{cyl} A$, that is,

$$
\begin{aligned}
S(A, r)=\{ & \exists E \subset \operatorname{cyl} A \cap \mathcal{V}_{2}(\operatorname{hyp} A, r),|E|<\infty, \forall e \in E \quad \omega(e)=0, \\
& E \text { separates } \infty \text { in cyl } A\} .
\end{aligned}
$$

Lemma 4.10. - Let $F$ be $a(d-1)$-dimensional set in $\Omega$ such that $\mathcal{H}^{d-2}(\partial F)<\infty$. We define

$$
\text { wall }(F, n)=S(F, f(n) / n) \cap
$$

$\left\{\right.$ all the edges in $\mathcal{V}_{2}(\operatorname{cyl} \partial F, 2 d / n) \cap \mathcal{V}_{2}($ hyp $F, f(n) / n)$ are closed $\}$.

Then

$$
\liminf _{n \rightarrow \infty} \frac{1}{n^{d-1}} \log \Phi_{n}[\operatorname{wall}(F, n)] \geqslant-\mathcal{H}^{d-1}(F) \tau(\text { nor } F) .
$$

Proof. - The number of edges in the set $\mathcal{V}_{2}(\operatorname{cyl} \partial F, 2 d / n) \cap \mathcal{V}_{2}$ (hyp $F, f(n) / n$ ) is less than $c(d) \mathcal{H}^{d-2}(\partial F) f(n) n^{d-2}$ for some positive constant $c(d)$ depending only on the dimension $d$. By the FKG inequality,

$$
\Phi_{n}[\operatorname{wall}(F, n)] \geqslant \Phi_{n}[S(F, f(n) / n)] \exp \left(c(d) \mathcal{H}^{d-2}(\partial F) f(n) n^{d-2} \ln (1-p)\right)
$$

and by using Lemma 4.7 of [15] we are done.

In view of the approximation result stated in Theorem 3.5, to prove the LDP lower bound we need only to show that for any $\varepsilon>0$, for any polyhedral element $(\mathcal{A}, \mathcal{T})$ in $\operatorname{TP}(\Omega)$ satisfying $\mathcal{H}^{d-1}\left(\overline{\partial^{*}(\mathcal{A}, \mathcal{T})} \cap \Gamma\right)<\varepsilon$, we have for all $\delta>0$,

$$
\liminf _{n \rightarrow \infty} \frac{1}{n^{d-1}} \log \Phi_{n}\left[\operatorname{Dist}\left(\left(\mathcal{A}_{n}, \mathcal{T}_{n}\right),(\mathcal{A}, \mathcal{T})\right)<\delta\right] \geqslant-\mathcal{I}(\mathcal{A}, \mathcal{T})-c(d, p, q) \varepsilon,
$$

where $c(d, p, q)$ is a constant depending on $d, p, q$ only. Let $\varepsilon>0$, let $(\mathcal{A}, \mathcal{T})$ be a polyhedral element of $\operatorname{TP}(\Omega)$ such that $\mathcal{H}^{d-1}\left(\overline{\partial^{*}(\mathcal{A}, \mathcal{T})} \cap \Gamma\right)<\varepsilon$ and let $\delta>0$. Up to a slight modification of the approximation procedure, we might assume that the sets $A_{1}, \ldots, A_{r}$ of $\mathcal{A}$ are connected and that they touch only a bounded number of boundary pieces. More precisely, we suppose that there exists $M \in \mathbb{N}$ such that

$$
\forall m \geqslant M \quad \forall i \in\{1, \ldots, r\} \quad \mathcal{T}\left(A_{i}, m\right)=0 .
$$

We suppose also that for any $i \in\{1, \ldots, r\}$ and any $m \in\{1, \ldots, M-1\}$, if $\mathcal{T}\left(A_{i}, m\right)=1$ then $\partial A_{i} \cap \Gamma^{m}$ is a relatively open subset of $\Gamma$. In particular, we have

$$
\mathcal{H}^{d-1}\left(\bigcup_{m>M} \Gamma^{m}\right)<\varepsilon
$$


By definition of a polyhedral element, $\partial^{*}(\mathcal{A}, \mathcal{T}) \cap \Omega$ is the union of a finite number of $(d-1)$-dimensional sets $F_{1}, \ldots, F_{s}$. Thus

$$
\mathcal{I}(\mathcal{A}, \mathcal{T}) \leqslant \sum_{1 \leqslant j \leqslant s} \mathcal{H}^{d-1}\left(F_{j}\right) \tau\left(\text { nor } F_{j}\right)+\varepsilon \tau_{\max } .
$$

Moreover, for each $i$ in $\{1, \ldots, s\}$, the relative boundary $\partial F_{i}$ has finite $(d-2)$ dimensional Hausdorff measure (we can achieve this by a slight perturbation of the polyhedral sets if necessary, using the hypothesis on $\Gamma$ ). Let $\delta>0$ and let $U_{1}, \ldots, U_{r}$ and $O_{1}, \ldots, O_{r}$ be open connected sets such that for any $i \in\{1, \ldots, r\}$

$$
U_{i} \subseteq O_{i} \subseteq A_{i}, \quad \mathrm{~d}_{2}\left(U_{i}, \Omega \backslash O_{i}\right)>0, \quad \mathrm{~d}_{2}\left(O_{i}, \Omega \backslash A_{i}\right)>0, \quad \operatorname{dist}_{L^{1}}\left(U_{i}, A_{i}\right)<\delta /\left(3 r^{2}\right)
$$

and finally for every $m \in\{1, \ldots, M\}$,

$$
\mathcal{T}\left(A_{i}, m\right)=1 \Rightarrow \partial U_{i} \cap \Gamma^{m} \neq \emptyset, \quad \mathcal{T}\left(A_{i}, m\right)=0 \Rightarrow \partial O_{i} \cap \Gamma^{m}=\emptyset .
$$

By hypothesis, the relative boundary of $\bigcup_{m} \Gamma^{m}$ in $\Gamma$ has zero $\mathcal{H}^{d-1}$ measure. By the outer regularity of $\mathcal{H}^{d-1}$ restricted to $\Gamma$, there exists an open set $O$ such that

$$
\overline{\bigcup_{m>M} \Gamma^{m}} \cup\left(\overline{\partial^{*}(\mathcal{A}, \mathcal{T})} \cap \Gamma\right) \subset O, \quad \mathcal{H}^{d-1}(O \cap \Gamma)<2 \varepsilon .
$$

Let us define

$$
\begin{aligned}
\eta & =\min \left(\mathrm{d}_{2}\left(\overline{\bigcup_{m>M} \Gamma^{m}} \cup\left(\overline{\partial^{*}(\mathcal{A}, \mathcal{T})} \cap \Gamma\right), \Gamma \backslash O\right), \min _{1 \leqslant i \leqslant r} d_{2}\left(O_{i}, \Omega \backslash A_{i}\right)\right), \\
U & =\mathcal{V}_{2}\left(\overline{\bigcup_{m>M} \Gamma^{m}} \cup\left(\overline{\partial^{*}(\mathcal{A}, \mathcal{T})} \cap \Gamma\right), \eta / 2\right) .
\end{aligned}
$$

Then $U$ is an open set such that

$$
\overline{\bigcup_{m>M} \Gamma^{m}} \cup\left(\overline{\partial^{*}(\mathcal{A}, \mathcal{T})} \cap \Gamma\right) \subseteq U, \quad \bar{U} \subseteq O, \quad \mathcal{H}^{d-1}(\bar{U} \cap \Gamma)<2 \varepsilon .
$$

Let $E_{n}$ be the event that every edge having an endpoint in $\mathcal{V}_{2}(\bar{U} \cap \Gamma, 2 / n)$ is closed. The number $N_{n}$ of all such edges can be estimated as follows. Since $\bar{U} \cap \Gamma$ is closed and $(d-1)$-rectifiable, we have (see the appendix)

$$
\lim _{h \rightarrow 0}(1 / 2 h) \mathcal{L}^{d}\left(\mathcal{V}_{2}(\bar{U} \cap \Gamma, h)\right)=\mathcal{H}^{d-1}(\bar{U} \cap \Gamma)<2 \varepsilon .
$$

Since

$$
N_{n} \leqslant 2 d\left|\mathbb{Z}_{n}^{d} \cap \mathcal{V}_{2}(\bar{U} \cap \Gamma, 2 / n)\right| \leqslant 2 d n^{d} \mathcal{L}^{d}\left(\mathcal{V}_{2}(\bar{U} \cap \Gamma, 4 / n)\right),
$$

we see that

$$
\limsup _{n \rightarrow \infty} n^{-(d-1)} N_{n} \leqslant 32 d \varepsilon
$$


If $E_{n}$ occurs then clearly there is no open edge exiting from

$$
\left(\bigcup_{m>M} \Gamma_{n}^{m}\right) \cup \mathcal{V}_{2}\left(\overline{\partial^{*}(\mathcal{A}, \mathcal{T})} \cap \Gamma, \eta / 4\right)
$$

Suppose that all the events

$$
E_{n}, \quad \text { full }\left(O_{i}, U_{i}, n\right), \quad 1 \leqslant i \leqslant r, \quad \text { wall }\left(F_{j}, n\right), \quad 1 \leqslant j \leqslant s
$$

occur simultaneously. Let us denote by $C_{i}$ the open cluster of the configuration containing the cluster realizing the event full $\left(O_{i}, U_{i}, n\right)$ and let $C_{i}^{\mathrm{FK}}$ be the FK cluster containing it. We have then that $\Omega_{n}^{\prime} \cap U_{i} \subseteq$ vor $C_{i}^{\mathrm{FK}}$. Moreover, the occurrence of the events $E_{n}$ and wall $\left(F_{j}, n\right), 1 \leqslant j \leqslant s$, precludes that an open path connects two distinct sets $O_{i}$ or two boundary pieces $\Gamma^{m}$ (apart from those which are already connected inside the sets $O_{i}$ ), thus the clusters $C_{i}^{\mathrm{FK}}$ are distinct and satisfy for $i=1, \ldots, r$,

$$
\Omega_{n}^{\prime} \cap U_{i} \subseteq \Omega_{n}^{\prime} \cap \operatorname{vor} C_{i}^{\mathrm{FK}} \subseteq \Omega_{n}^{\prime} \backslash \bigcup_{k \neq i} O_{k}
$$

Therefore,

$$
\begin{aligned}
\sum_{i=1}^{r} \operatorname{dist}_{L^{1}}\left(\operatorname{vor} C_{i}^{\mathrm{FK}}, A_{i}\right) & \leqslant 2 \mathcal{L}^{d}\left(\Omega \backslash \Omega_{n}^{\prime}\right)+\sum_{i=1}^{r} \mathcal{L}^{d}\left(\Omega_{n}^{\prime} \backslash\left(U_{i} \cup \bigcup_{k \neq i} O_{k}\right)\right) \\
& \leqslant 2 \mathcal{L}^{d}\left(\Omega \backslash \Omega_{n}^{\prime}\right)+\sum_{i=1}^{r} \sum_{k=1}^{r} \mathcal{L}^{d}\left(A_{k} \backslash U_{k}\right)<2 \mathcal{L}^{d}\left(\Omega \backslash \Omega_{n}^{\prime}\right)+\delta / 3 .
\end{aligned}
$$

Moreover, the condition imposed on the sets $O_{i}$, together with the definition of full $\left(O_{i}, U_{i}, n\right)$, ensures that $\mathcal{T}\left(\operatorname{vor} C_{i}^{\mathrm{FK}}, m\right)=\mathcal{T}\left(A_{i}, m\right)$ for each $i=1, \ldots, r$ and $m=$ $1, \ldots, M$. Necessarily, for any $C^{\mathrm{FK}} \in \mathcal{C}_{n}^{\mathrm{FK}} \backslash\left\{C_{1}^{\mathrm{FK}}, \ldots, C_{r}^{\mathrm{FK}}\right\}, \Omega_{n}^{\prime} \cap \operatorname{vor} C^{\mathrm{FK}} \subseteq \Omega_{n}^{\prime} \backslash$ $\bigcup_{1 \leqslant i \leqslant r} U_{i}$, and therefore

$$
\sum_{C^{\mathrm{FK}} \in \mathcal{C}_{n}^{\mathrm{FK}} \backslash\left\{C_{1}^{\mathrm{FK}}, \ldots, C_{r}^{\mathrm{FK}}\right\}} \mathcal{L}^{d}\left(\operatorname{vor} C^{\mathrm{FK}}\right)<\delta / 3+\mathcal{L}^{d}\left(\Omega \backslash \Omega_{n}^{\prime}\right),
$$

whence also $\operatorname{Dist}\left(\left(\mathcal{A}_{n}, \mathcal{T}_{n}\right),(\mathcal{A}, \mathcal{T})\right)<\delta$ for $n$ large enough so that $\mathcal{L}^{d}\left(\Omega \backslash \Omega_{n}^{\prime}\right)<\delta / 9$. This discussion shows that

$$
\Phi_{n}\left[\operatorname{Dist}\left(\left(\mathcal{A}_{n}, \mathcal{T}_{n}\right),(\mathcal{A}, \mathcal{T})\right)<\delta\right] \geqslant \Phi_{n}\left[\bigcap_{1 \leqslant i \leqslant r} \operatorname{full}\left(O_{i}, U_{i}, n\right) \cap E_{n} \cap \bigcap_{1 \leqslant j \leqslant s} \operatorname{wall}\left(F_{j}, n\right)\right] .
$$

The event $E_{n}$ depends on the edges inside $\mathcal{V}_{2}(\bar{U} \cap \Gamma, 3 / n)$, the event wall $\left(F_{j}, n\right)$ depends on the edges inside $\mathcal{V}_{2}\left(F_{j}, f(n) / n\right)$, whereas full $\left(O_{i}, U_{i}, n\right)$ depends on the edges inside $O_{i}$. Because of the condition imposed on $\eta$, we have

$$
d_{2}\left(\mathcal{V}_{2}(\bar{U} \cap \Gamma, 3 / n), O_{1} \cup \cdots \cup O_{r}\right)>0 .
$$

The distance between the sets $F_{1} \cup \cdots \cup F_{s}$ and $O_{1} \cup \cdots \cup O_{r}$ is also strictly positive. Since $f(n) / n \rightarrow 0$ as $n \rightarrow \infty$, Lemma 4.9 implies that 


$$
\liminf _{n \rightarrow \infty} \frac{1}{n^{d-1}} \log \Phi_{n}\left[\bigcap_{1 \leqslant i \leqslant r} \operatorname{full}\left(O_{i}, U_{i}, n\right) \mid E_{n} \cap \bigcap_{1 \leqslant j \leqslant s} \operatorname{wall}\left(F_{j}, n\right)\right]=0 .
$$

By the FKG inequality, Lemma 4.10, and the previous estimates, we obtain

$$
\begin{aligned}
& \liminf _{n \rightarrow \infty} \frac{1}{n^{d-1}} \log \Phi_{n}\left[E_{n} \cap \bigcap_{1 \leqslant j \leqslant s} \operatorname{wall}\left(F_{j}, n\right)\right] \\
& \quad \geqslant \liminf _{n \rightarrow \infty} \frac{1}{n^{d-1}} \log \left(\frac{p}{p+q(1-p)}\right)^{N_{n}}-\sum_{1 \leqslant j \leqslant s} \mathcal{H}^{d-1}\left(F_{j}\right) \tau\left(\text { nor } F_{j}\right) \\
& \geqslant 32 d \varepsilon \log \frac{p}{p+q(1-p)}-\mathcal{I}(\mathcal{A}, \mathcal{T})-\varepsilon \tau_{\max }
\end{aligned}
$$

which yields the desired lower bound.

\subsection{Proof of Theorem 1.1 (Potts model)}

Throughout the proof, we work with the coupling measure $\mathbb{P}_{n}$ between the FK measure $\Phi_{n}$ and the Potts measure $\mu_{n}$ (see Section 2.2). For a given $n$, we express the local average of the $i$ th color $\sigma_{n}(x, i)$ with the help of the FK representation: for each $x \in \Omega$ and each color $i=1, \ldots, q$,

$$
\sigma_{n}(x, i)=f(n)^{-d} \sum_{C \text { cluster, } \sigma(C)=i}|C \cap \Lambda(x, f(n) / n)|,
$$

where the sum runs over all the open clusters of the configuration. We separate the contribution of the small and large clusters by setting

$$
\sigma_{n}^{\text {small }}(x, i)=f(n)^{-d} \sum_{C, \operatorname{diam} C \leqslant f(n), \sigma(C)=i}|C \cap \Lambda(x, f(n) / n)|,
$$

where the sum runs now over all the small open clusters of the configuration; and we define $\sigma_{n}^{\text {large }}$ analogously except that the sum is running over the large clusters (that is the elements of $\mathcal{C}_{n}$ ) colored with $i$.

LEMMA 4.11. - For any $\delta>0$,

$$
\limsup _{n \rightarrow \infty} \frac{1}{n^{d-1}} \log \mathbb{P}_{n}\left[\sum_{i=1, \ldots, q} \int_{\Omega}\left|\sigma_{n}^{\text {small }}(x, i)-(1-\theta) / q\right| \mathrm{d} x \geqslant \delta\right]=-\infty .
$$

Proof. - The proof is based on the observation that the i.i.d. coloring of the small FK clusters (whose number is of volume order) will create fluctuations whose large deviations behavior is close to volume order. We omit a full blown proof since it is a straightforward adaptation of the proof of Lemma 7.10 in [15].

For $i=1, \ldots, q$, we set

$$
D_{n}^{i}=\bigcup_{C \in \mathcal{C}_{n}, \sigma(C)=i} \operatorname{vor} C
$$


Notice that all the clusters belonging to the same FK cluster have necessarily the same color. Thus, by the very definition of the sets $\operatorname{vor} C^{\mathrm{FK}}, C^{\mathrm{FK}} \in \mathcal{C}_{n}^{\mathrm{FK}}$, we have

$$
D_{n}^{i}=\bigcup_{C^{\mathrm{FK}} \in \mathcal{C}_{n}^{\mathrm{FK}}, C^{\mathrm{FK}} \subseteq D_{n}^{i}} \operatorname{vor} C^{\mathrm{FK}} .
$$

LEMMA 4.12. - For $\delta>0$,

$$
\begin{aligned}
\limsup _{n \rightarrow \infty} \frac{1}{n^{d-1}} \log \mathbb{P}_{n}[ & \sum_{i=1, \ldots, q} \int_{D_{n}^{i}}\left(\left|\sigma_{n}^{\text {large }}(x, i)-\theta\right|\right. \\
& \left.\left.+\sum_{\substack{j=1, \ldots, q \\
j \neq i}} \sigma_{n}^{\text {large }}(x, j)\right) \mathrm{d} x>\delta\right]=-\infty .
\end{aligned}
$$

Proof. - We work with the $(K=f(n), \alpha=2)$ renormalization process with block variables $X(\underline{x})$ given by the indicator function of the event $T\left(B^{\prime}(\underline{x}), f(n), \delta\right)^{c}$. Suppose that $X(\underline{x})=0$, i.e., the block is good. Let $x \in B(\underline{x})$. Then there exists exactly one large cluster $C$ such that $\mathrm{d}_{\infty}(x, C) \leqslant f(n)$ so that $x \in \operatorname{vor} C$ and also to $D_{n}^{\sigma(C)}$. This cluster satisfies in addition

$$
|| C \cap \Lambda(x, f(n) / n)\left|-\theta f(n)^{d}\right|<\delta f(n)^{d}
$$

therefore $\left|\sigma_{n}^{\text {large }}(x, \sigma(C))-\theta\right|<\delta$ and for each $j \neq \sigma(C), \sigma_{n}^{\text {large }}(x, j)=0$. We split the regions of integration $D_{n}^{i}$ into the blocks $B(\underline{x}), \underline{x} \in \underline{\Omega}_{n}$, and we use the previous inequality to get

$$
\begin{gathered}
\sum_{i=1, \ldots, q} \int_{D_{n}^{i}}\left(\left|\sigma_{n}^{\text {large }}(x, i)-\theta\right|+\sum_{\substack{j=1, \ldots, q \\
j \neq i}} \sigma_{n}^{\text {large }}(x, j)\right) \mathrm{d} x \\
\leqslant 2(f(n) / n)^{d} \sum_{\underline{x} \in \underline{\Omega}_{n}} X(\underline{x})+\delta \mathcal{L}^{d}(\Omega)+\mathcal{L}^{d}\left(\Omega \backslash \Omega_{n}^{\prime}\right) .
\end{gathered}
$$

By the estimate (16) the block process satisfies (18) with $\varepsilon=b \exp (-c f(n))$. The result follows from Lemma 2.3.

We next compare the random partitions $\left(D_{n}^{1}, \ldots, D_{n}^{q}\right)$ and $\left(A_{n}^{0}, A_{n}^{1}, \ldots, A_{n}^{q}\right)$.

LEMMA 4.13. - For any $\delta>0$,

$$
\limsup _{n \rightarrow \infty} \frac{1}{n^{d-1}} \log \mathbb{P}_{n}\left[\mathcal{L}^{d}\left(A_{n}^{0}\right)+\sum_{i=1, \ldots, q} \operatorname{dist}_{L^{1}}\left(A_{n}^{i}, D_{n}^{i}\right) \geqslant \delta\right]=-\infty .
$$

Proof. - For $\varepsilon>0$, we denote by $D_{n}^{i}(\varepsilon)$ the set of points $x \in D_{n}^{i}$ for which

$$
\begin{aligned}
& \sum_{\substack{j=1, \ldots, q \\
j \neq i}}\left(\left|\sigma_{n}^{\text {small }}(x, j)-(1-\theta) / q\right|+\sigma_{n}^{\text {large }}(x, j)\right) \\
& \quad+\left|\sigma_{n}^{\text {small }}(x, i)-(1-\theta) / q\right|+\left|\sigma_{n}^{\text {large }}(x, i)-\theta\right|<\varepsilon .
\end{aligned}
$$


If $x$ belongs to $D_{n}^{i}(\varepsilon)$ then

$$
\left|\sigma_{n}(x, i)-(\theta+(1-\theta) / q)\right|<\varepsilon, \quad \forall j \neq i \quad\left|\sigma_{n}(x, j)-(1-\theta) / q\right|<\varepsilon .
$$

Therefore, for $\varepsilon$ small enough we have $D_{n}^{i}(\varepsilon) \subseteq A_{n}^{i}$. Since $D_{n}^{1}, \ldots, D_{n}^{q}$ is a partition of $\Omega$, we see that

$$
\begin{aligned}
& \mathcal{L}^{d}\left(A_{n}^{0}\right)+\sum_{i=1, \ldots, q} \operatorname{dist}_{L^{1}}\left(A_{n}^{i}, D_{n}^{i}\right) \\
& \quad \leqslant \mathcal{L}^{d}\left(\bigcup_{i=1, \ldots, q} D_{n}^{i} \backslash D_{n}^{i}(\varepsilon)\right)+2 \sum_{i=1, \ldots, q} \mathcal{L}^{d}\left(D_{n}^{i} \backslash D_{n}^{i}(\varepsilon)\right) \leqslant 3 \sum_{i=1, \ldots, q} \mathcal{L}^{d}\left(D_{n}^{i} \backslash D_{n}^{i}(\varepsilon)\right) .
\end{aligned}
$$

Lemmas 4.11 and 4.12 yield that for any $\delta>0$

$$
\limsup _{n \rightarrow \infty} \frac{1}{n^{d-1}} \log \mathbb{P}_{n}\left[\sum_{i=1, \ldots, q} \mathcal{L}^{d}\left(D_{n}^{i} \backslash D_{n}^{i}(\varepsilon)\right)>\delta\right]=-\infty
$$

which, together with the previous inequality, concludes the proof.

Lemmas 4.11-4.13 together yield the claim of Theorem 1.1.

\subsection{Asymptotics of the conditioning event}

In order to transfer the LDP from the FK model to the Potts model, we will need to estimate the probability of the conditioning event. This is the purpose of the next lemma.

LEMmA 4.14. - Let $\mathcal{F}$ denote the set of the t-partitions such that no set touches simultaneously two distinct boundary parts, i.e.,

$$
\mathcal{F}=\left\{(\mathcal{A}, \mathcal{T}) \in \operatorname{TP}(\Omega) ; \forall A \in \mathcal{A} \sum_{\substack{1 \leqslant i, j \leqslant q \\ i \neq j}} \mathcal{T}(A, i) \mathcal{T}(A, j)=0\right\} .
$$

We have

$$
\begin{aligned}
& \lim _{n \rightarrow \infty} \frac{1}{n^{d-1}} \log \Phi_{n}\left[\text { there is no connection between } \Gamma_{n}^{i} \text { and } \Gamma_{n}^{j} \text { for } i \neq j, 1 \leqslant i, j \leqslant q\right] \\
& \quad=-\min _{\mathcal{F}} \mathcal{I}=-\min _{P(\Omega, q)} \mathcal{I} .
\end{aligned}
$$

Remark. - Notice that the event estimated above is the absence of any open path between boundary pieces, even of open paths of diameter less than $f(n)$. This event cannot be expressed directly with the random t-partition $\left(\mathcal{A}_{n}, \mathcal{T}_{n}\right)$.

Proof. - The final equality $\min _{\mathcal{F}} \mathcal{I}=\min _{P(\Omega, q)} \mathcal{I}$ is proved in Lemma 1.7. We turn now to the computation of the limit presented in the statement of the lemma. We first prove that the set $\mathcal{F}$ is closed in $(\operatorname{TP}(\Omega)$, Dist). Indeed, if $(\mathcal{A}, \mathcal{T}) \in \overline{\mathcal{F}}$, then there exists a sequence $\left(\mathcal{A}_{n}, \mathcal{T}_{n}\right)_{n \in \mathbb{N}}$ in $\mathcal{F}$ which converges to $(\mathcal{A}, \mathcal{T})$. Let $A$ belong to $\mathcal{A}$. There exists a sequence $\left(A_{n}\right)_{n \in \mathbb{N}}$ such that for each $n \in \mathbb{N}, A_{n}$ belongs to $\mathcal{A}_{n}$ and

$$
\lim _{n \rightarrow \infty} \operatorname{dist}_{L^{1}}\left(A, A_{n}\right)+\sum_{i=1, \ldots, q} 2^{-i}\left|\mathcal{T}_{n}\left(A_{n}, i\right)-\mathcal{T}(A, i)\right|=0 .
$$


Since $\left(\mathcal{A}_{n}, \mathcal{T}_{n}\right) \in \mathcal{F}$, then $\mathcal{T}_{n}\left(A_{n}, i\right) \mathcal{T}_{n}\left(A_{n}, j\right)=0$ for $i, j \in\{1, \ldots, q\}, i \neq j$. Sending $n$ to $\infty$, we obtain that $\mathcal{T}(A, i) \mathcal{T}(A, j)=0$ for different colors $i, j$, thus $(\mathcal{A}, \mathcal{T})$ belongs to $\mathcal{F}$ as claimed.

Our next observation is that $\stackrel{\circ}{\mathcal{F}}$ (the interior of $\mathcal{F}$ ) can be written as

$$
\mathcal{G}=\left\{(\mathcal{A}, \mathcal{T}) \in \mathcal{F} ; \sum_{A \in \mathcal{A}} \sum_{i=1, \ldots, q} \mathcal{T}(A, i) \geqslant q-1\right\} .
$$

We prove first that $\stackrel{\mathcal{F}}{\subseteq} \mathcal{G}$. Let $(\mathcal{A}, \mathcal{T}) \in \mathcal{F}$ be such that

$$
\sum_{A \in \mathcal{A}} \sum_{i=1, \ldots, q} \mathcal{T}(A, i)<q-1
$$

Then there exist two distinct colors $k, l$ such that $\sum_{A \in \mathcal{A}} \mathcal{T}(A, k)+\mathcal{T}(A, l)=0$. For every $n$ large enough let $B_{n}$ be a ball of radius $1 / n$ included in $\Omega$. Let us define

$$
\begin{gathered}
\mathcal{A}_{n}=\left\{A \backslash B_{n} ; A \in \mathcal{A}\right\} \cup\left\{B_{n}\right\}, \\
\forall A \in \mathcal{A} \quad \mathcal{T}_{n}\left(A \backslash B_{n}, \cdot\right)=\mathcal{T}(A, \cdot), \\
\forall j \in\{1, \ldots, q\} \quad \mathcal{T}_{n}\left(B_{n}, j\right)= \begin{cases}1 ; & j=k \text { or } j=l, \\
0 ; & \text { otherwise. }\end{cases}
\end{gathered}
$$

Clearly $\left(\mathcal{A}_{n}, \mathcal{T}_{n}\right) \notin \mathcal{F}$, however, $\operatorname{Dist}\left((\mathcal{A}, \mathcal{T}),\left(\mathcal{A}_{n}, \mathcal{T}_{n}\right)\right) \leqslant 2 \mathcal{L}^{d}\left(B_{n}\right)$. Therefore $\left(\mathcal{A}_{n}, \mathcal{T}_{n}\right)_{n \in \mathbb{N}}$ is a sequence in $\operatorname{TP}(\Omega) \backslash \mathcal{F}$ converging towards $(\mathcal{A}, \mathcal{T})$. Hence $(\mathcal{A}, \mathcal{T})$ is not in $\mathcal{F}$ and therefore $\mathcal{\mathcal { F }} \subseteq \mathcal{G}$.

To show the other inclusion $\mathcal{G} \subset \mathcal{F}$ we start by considering an element $(\mathcal{A}, \mathcal{T})$ of $\mathcal{G}$. There exists at most one color $i_{0}$ such that $\sum_{A \in \mathcal{A}} \mathcal{T}\left(A, i_{0}\right)=0$. For each color $i \neq i_{0}$ there exists a unique set $A_{i} \in \mathcal{A}$ such that $\mathcal{T}\left(A_{i}, i\right)=1$. Pick $\varepsilon>0$ such that

$$
\varepsilon<\min \left(2^{-q},(1 / 2) \min _{i \neq i_{0}} \mathcal{L}^{d}\left(A_{i}\right)\right)
$$

and let $\left(\mathcal{A}^{\prime}, \mathcal{T}^{\prime}\right) \in \operatorname{TP}(\Omega)$ be such that $\operatorname{Dist}\left((\mathcal{A}, \mathcal{T}),\left(\mathcal{A}^{\prime}, \mathcal{T}^{\prime}\right)\right)<\varepsilon$. Necessarily, for each color $i \neq i_{0}$ there exists a set $A_{i}^{\prime} \in \mathcal{A}^{\prime}$ satisfying

$$
\operatorname{dist}_{L^{1}}\left(A_{i}, A_{i}^{\prime}\right)+\sum_{j=1, \ldots, q} 2^{-j}\left|\mathcal{T}\left(A_{i}, j\right)-\mathcal{T}\left(A_{i}^{\prime}, j\right)\right|<\varepsilon .
$$

The condition imposed on $\varepsilon$ guarantees that the sets $A_{i}^{\prime}, i \neq i_{0}$, are distinct and also that, if $i \neq i_{0}$, we have $\mathcal{T}\left(A_{i}, j\right)=\mathcal{T}\left(A_{i}^{\prime}, j\right)$ for each color $j$. Since at most one set of $\mathcal{A}^{\prime}$ can touch any boundary piece, we conclude that $\left(\mathcal{A}^{\prime}, \mathcal{T}^{\prime}\right) \in \mathcal{F}$ and therefore $\mathcal{G} \subseteq \mathcal{F}$.

We show finally that

$$
\inf \{\mathcal{I}(\mathcal{A}, \mathcal{T}) ;(\mathcal{A}, \mathcal{T}) \in \stackrel{\circ}{\mathcal{F}}\}=\inf \{\mathcal{I}(\mathcal{A}, \mathcal{T}) ;(\mathcal{A}, \mathcal{T}) \in \mathcal{F}\}
$$

Let $(\mathcal{A}, \mathcal{T}) \in \mathcal{F} \backslash \dot{\mathcal{F}}$. Then

$$
\sum_{A \in \mathcal{A}} \sum_{i=1, \ldots, q} \mathcal{T}(A, i)<q-1
$$


Let $l=q-\sum_{A \in \mathcal{A}} \sum_{i=1, \ldots, q} \mathcal{T}(A, i)$ and let $i_{1}, \ldots, i_{l}$ be the $l$ distinct colors such that

$$
\sum_{A \in \mathcal{A}}\left(\mathcal{T}\left(A, i_{1}\right)+\cdots+\mathcal{T}\left(A, i_{l}\right)\right)=0 .
$$

Let $B_{1}(n), \ldots, B_{l}(n)$ be $l$ disjoint balls of radius $1 / n$ included in $\Omega$ (we assume that $n$ is large enough) and let $\left(\mathcal{A}_{n}, \mathcal{T}_{n}\right)$ be defined by

$$
\begin{aligned}
& \mathcal{A}_{n}=\left\{A \backslash\left(B_{1}(n) \cup \cdots \cup B_{l}(n)\right) ; A \in \mathcal{A}\right\} \cup\left\{B_{1}(n), \ldots, B_{l}(n)\right\}, \\
& \forall A \in \mathcal{A} \quad \mathcal{T}_{n}\left(A \backslash\left(B_{1}(n) \cup \cdots \cup B_{l}(n)\right), \cdot\right)=\mathcal{T}(A, \cdot),
\end{aligned}
$$

and for each $i=1, \ldots, q$ and $j=1, \ldots, l$

$$
\mathcal{T}_{n}\left(B_{j}(n), i\right)= \begin{cases}1 ; & i=i_{j}, \\ 0 ; & \text { otherwise. }\end{cases}
$$

Then $\left(\mathcal{A}_{n}, \mathcal{T}_{n}\right)$ belongs to $\mathcal{F}$. Moreover,

$$
\mathcal{I}\left(\mathcal{A}_{n}, \mathcal{T}_{n}\right) \leqslant \mathcal{I}(\mathcal{A}, \mathcal{T})+l \tau_{\max } \alpha_{d-1} / n^{d-1}, \quad \operatorname{Dist}\left((\mathcal{A}, \mathcal{T}),\left(\mathcal{A}_{n}, \mathcal{T}_{n}\right)\right) \leqslant l \alpha_{d} / n^{d} .
$$

Sending $n$ to $\infty$ and taking the infimum over $(\mathcal{A}, \mathcal{T})$ in $\mathcal{F} \backslash \dot{\mathcal{F}}$, we obtain the desired inequality $\inf _{\mathcal{F} \backslash \mathcal{F}} \mathcal{I} \geqslant \inf _{\mathcal{F}} \mathcal{I}$.

Next, we claim that

$$
\stackrel{\circ}{\mathcal{F}} \subset\left\{\text { there is no connection between } \Gamma_{n}^{i} \text { and } \Gamma_{n}^{j} \text { for } i \neq j, 1 \leqslant i, j \leqslant q\right\} \subset \mathcal{F} .
$$

The second inclusion is straightforward. The first one stems from the fact that, on the event $\stackrel{\circ}{\mathcal{F}}$, there exist $q-1$ distinct FK clusters which are touching exactly one boundary piece, therefore no cluster can touch simultaneously two boundary pieces. The claim of the Lemma is obtained by applying the LDP principle for FK percolation stated in Theorem 1.8

$$
-\inf _{\mathcal{F}} \mathcal{I} \leqslant \liminf _{n \rightarrow \infty} \frac{1}{n^{d-1}} \log \Phi_{n}[\stackrel{\mathcal{F}}{ }] \leqslant \limsup _{n \rightarrow \infty} \frac{1}{n^{d-1}} \log \Phi_{n}[\mathcal{F}] \leqslant-\inf _{\mathcal{F}} \mathcal{I}
$$

in conjunction with the fact that $\inf _{\mathcal{F}} \mathcal{I}=\inf _{\mathcal{F}} \mathcal{I}$.

\subsection{Proof of the lower bound in Theorem 1.3 (Potts model)}

Let $\vec{A}=\left(A^{0}, \ldots, A^{q}\right)$ be an element of $P(\Omega, q)$ such that $\mathcal{I}(\vec{A})<\infty$. Let $\delta \in(0,1)$ and let $B_{1}, \ldots, B_{q}$ be $q$ disjoint balls of volume $\delta /\left(8 q^{2}\right)$ included in $\Omega$ (we assume that $\delta$ is sufficiently small). For $i=1, \ldots, q$, we set

$$
E^{i}=\left(A^{i} \backslash \bigcup_{j=1, \ldots, q} B_{j}\right) \cup B_{i}
$$

By Lemma 4.13, we have 


$$
\begin{aligned}
\liminf _{n \rightarrow \infty} & \frac{1}{n^{d-1}} \log \mu_{n}\left[\operatorname{dist}_{P}\left(\left(A_{n}^{0}, \ldots, A_{n}^{q}\right), \vec{A}\right)<\delta\right] \\
\geqslant & \liminf _{n \rightarrow \infty} \frac{1}{n^{d-1}} \log \mathbb{P}_{n}\left[\sum_{i=1, \ldots, q} \operatorname{dist}_{L^{1}}\left(A^{i}, D_{n}^{i}\right)<\delta / 2\right] \\
\geqslant & \liminf _{n \rightarrow \infty} \frac{1}{n^{d-1}} \log \mathbb{P}_{n}\left[\sum_{i=1, \ldots, q} \operatorname{dist}_{L^{1}}\left(E^{i}, D_{n}^{i}\right)<\delta / 4\right] .
\end{aligned}
$$

Furthermore, recalling the definition of $D_{n}^{i}$, we observe that

$$
\begin{aligned}
\mathbb{P}_{n}[ & \left.\sum_{i=1, \ldots, q} \operatorname{dist}_{L^{1}}\left(E^{i}, D_{n}^{i}\right)<\delta / 4\right] \\
\geqslant & \mathbb{P}_{n}\left[\exists C_{1}^{\mathrm{FK}}, \ldots, C_{q}^{\mathrm{FK}} \in \mathcal{C}_{n}^{\mathrm{FK}} \quad \forall i=1, \ldots, q \quad \sigma\left(C_{i}^{\mathrm{FK}}\right)=i \quad\right. \text { and } \\
& \left.\operatorname{dist}_{L^{1}}\left(\operatorname{vor} C_{i}^{\mathrm{FK}}, E^{i}\right)<\delta /(8 q)\right] \\
\geqslant & \frac{\Phi_{n}\left[\operatorname{Dist}\left(\left(\mathcal{A}_{n}, \mathcal{T}_{n}\right),(\mathcal{A}, \mathcal{T})\right)<\delta /\left(8 q 2^{q}\right) \text { and no connection between } \Gamma_{n}^{1}, \ldots, \Gamma_{n}^{q}\right]}{\Phi_{n}\left[\text { no connection between } \Gamma_{n}^{1}, \ldots, \Gamma_{n}^{q}\right]},
\end{aligned}
$$

where $(\mathcal{A}, \mathcal{T})$ is the t-partition given by $\mathcal{A}=\left\{E^{1}, \ldots, E^{q}\right\}$ and

$$
\mathcal{T}\left(E^{i}, j\right)= \begin{cases}1 ; & j=i \\ 0 ; & j \neq i\end{cases}
$$

Suppose that the event $\left\{\operatorname{Dist}\left(\left(\mathcal{A}_{n}, \mathcal{T}_{n}\right),(\mathcal{A}, \mathcal{T})\right)<\delta /\left(8 q 2^{q}\right)\right\}$ occurs. Then there exist $q$ disjoint FK clusters $C_{1}^{\mathrm{FK}}, \ldots, C_{q}^{\mathrm{FK}}$ such that

$$
\sum_{1 \leqslant i \leqslant q} \operatorname{dist}_{L^{1}}\left(\operatorname{vor} C_{i}^{\mathrm{FK}}, E^{i}\right)+\sum_{1 \leqslant i \leqslant q} \sum_{1 \leqslant j \leqslant q} 2^{-j}\left|\mathcal{T}_{n}\left(\operatorname{vor} C_{i}^{\mathrm{FK}}, j\right)-\mathcal{T}\left(E^{i}, j\right)\right|<\delta /\left(8 q 2^{q}\right),
$$

which implies that $\mathcal{T}_{n}\left(\operatorname{vor} C_{i}^{\mathrm{FK}}, j\right)=\mathcal{T}\left(E^{i}, j\right)$ for all $i, j=1, \ldots, q$; in this situation, there is one FK cluster touching each boundary piece (we added the balls $B_{1}, \ldots, B_{q}$ to ensure this) and each of these clusters touches exactly one boundary piece, so that there is no connection between $\Gamma_{n}^{1}, \ldots, \Gamma_{n}^{q}$. Thus

$$
\mathbb{P}_{n}\left[\sum_{i=1, \ldots, q} \operatorname{dist}_{L^{1}}\left(E^{i}, D_{n}^{i}\right)<\delta / 4\right] \geqslant \frac{\Phi_{n}\left[\operatorname{Dist}\left(\left(\mathcal{A}_{n}, \mathcal{T}_{n}\right),(\mathcal{A}, \mathcal{T})\right)<\delta /\left(8 q 2^{q}\right)\right]}{\Phi_{n}\left[\text { no connection between } \Gamma_{n}^{1}, \ldots, \Gamma_{n}^{q}\right]}
$$

Since $\mathcal{I}(\mathcal{A}, \mathcal{T})=\mathcal{I}(\vec{A})$, the LD lower bound of Theorem 1.8 and Lemma 4.14 yield that for any $\delta>0$,

$$
\liminf _{n \rightarrow \infty} \frac{1}{n^{d-1}} \log \mu_{n}\left[\operatorname{dist}_{P}\left(\left(A_{n}^{0}, \ldots, A_{n}^{q}\right), \vec{A}\right)<\delta\right] \geqslant-\mathcal{I}(\vec{A})+\min _{P(\Omega, q)} \mathcal{I} .
$$

\subsection{Proof of the upper bound in Theorem 1.3 (Potts model)}

Let $\mathbb{E}$ be a closed subset of $(P(\Omega, q)$, dist $P)$. By Lemma 4.13 , we need only to show that 


$$
\limsup _{n \rightarrow \infty} \frac{1}{n^{d-1}} \log \mathbb{P}_{n}\left[\left(\emptyset, D_{n}^{1}, \ldots, D_{n}^{q}\right) \in \mathbb{E}\right] \leqslant-\inf \{\mathcal{I}(\vec{A}) ; \vec{A} \in \mathbb{E}\}+\min _{P(\Omega, q)} \mathcal{I} .
$$

Let $\mathcal{E}$ be the set of the elements $(\mathcal{A}, \mathcal{T})$ of $\operatorname{TP}(\Omega)$ such that $\mathcal{A}$ can be partitioned in $q$ collections of sets $\mathcal{A}^{1}, \ldots, \mathcal{A}^{q}$ satisfying

$$
\sum_{i=1, \ldots, q} \sum_{\substack{A \in \mathcal{A}^{i} \\ j}} \sum_{\substack{j=1, \ldots, q \\ j \neq i}} \mathcal{T}(A, j)=0 \quad \text { and } \quad\left(\emptyset, \bigcup_{A \in \mathcal{A}^{1}} A, \ldots, \bigcup_{A \in \mathcal{A}^{q}} A\right) \in \mathbb{E} .
$$

The definition of $D_{n}^{1}, \ldots, D_{n}^{q}$ implies that

$$
\begin{aligned}
\mathbb{P}_{n}\left[\left(\emptyset, D_{n}^{1}, \ldots, D_{n}^{q}\right) \in \mathbb{E}\right] & \leqslant \Phi_{n}^{\nless}\left[\left(\mathcal{A}_{n}, \mathcal{T}_{n}\right) \in \mathcal{E}\right] \\
& \leqslant \frac{\Phi_{n}\left[\left(\mathcal{A}_{n}, \mathcal{T}_{n}\right) \in \mathcal{E}\right]}{\Phi_{n}\left[\text { no connection between } \Gamma_{n}^{1}, \ldots, \Gamma_{n}^{q}\right]} .
\end{aligned}
$$

LEMMA 4.15. - The set $\mathcal{E}$ is a closed subset of $(\mathrm{TP}(\Omega)$, Dist).

Proof. - Let $\left(\mathcal{A}_{n}^{\prime}, \mathcal{T}_{n}^{\prime}\right)_{n \in \mathbb{N}}$ be a sequence in $\mathcal{E}$ converging towards an element $\left(\mathcal{A}^{\prime}, \mathcal{T}^{\prime}\right) \in$ $\operatorname{TP}(\Omega)$. Let $\left(\left(A^{\prime}(i), T^{\prime}(i, \cdot)\right), i \in \mathbb{N}\right),\left(\left(A_{n}^{\prime}(i), T_{n}^{\prime}(i, \cdot)\right), i \in \mathbb{N}\right)_{n \in \mathbb{N}}$ be arrangements of $\left(\mathcal{A}^{\prime}, \mathcal{T}^{\prime}\right)$ and $\left(\mathcal{A}_{n}^{\prime}, \mathcal{T}_{n}^{\prime}\right)_{n \in \mathbb{N}}$ such that

$$
\sum_{i \in \mathbb{N}}\left(\operatorname{dist}_{L^{1}}\left(A^{\prime}(i), A_{n}^{\prime}(i)\right)+\sum_{j=1, \ldots, q} 2^{-j}\left|T^{\prime}(i, j)-T_{n}^{\prime}(i, j)\right|\right)
$$

converges to 0 as $n \rightarrow \infty$. By the definition of $\mathcal{E}$, for each $n \in \mathbb{N}$, there exist $q$ disjoint sets of indices $I_{1, n}^{\prime}, \ldots, I_{q, n}^{\prime}$ such that

$$
\begin{aligned}
& \text { (i) } \sum_{i=1, \ldots, q} \sum_{j \in I_{i, n}^{\prime}} \sum_{\substack{k=1, \ldots, q \\
k \neq i}} T_{n}^{\prime}(j, k)=0, \\
& \text { (ii) } \bigcup_{i=1, \ldots, q} I_{i, n}^{\prime}=\left\{j \in \mathbb{N} ; A_{n}^{\prime}(j) \neq \emptyset\right\}, \\
& \text { (iii) } \quad\left(\emptyset, \bigcup_{j \in I_{1, n}^{\prime}} A_{n}^{\prime}(j), \ldots, \bigcup_{j \in I_{q, n}^{\prime}} A_{n}^{\prime}(j)\right) \in \mathbb{E} .
\end{aligned}
$$

Let $\phi_{n}: \mathbb{N} \rightarrow\{0,1, \ldots, q\}$ be defined by

$$
\phi_{n}(j)= \begin{cases}i ; & j \in I_{i, n}^{\prime}, i=1, \ldots, q \\ 0 ; & \text { otherwise }\end{cases}
$$

By passing to a subsequence if necessary, we can assume that the limit

$$
\lim _{n \rightarrow \infty} \phi_{n}(j)=\phi(j) \in\{0, \ldots, q\}
$$

exists for $j \in \mathbb{N}$. Obviously, $\phi(j) \neq 0$ whenever $A^{\prime}(j) \neq 0$. We set for $i=1, \ldots, q$

$$
I_{i}^{\prime}=\left\{j \in \mathbb{N} ; A^{\prime}(j) \neq \emptyset, \phi(j)=i\right\} .
$$


Let $M \in \mathbb{N}$ be fixed. We have for each color $i, \lim _{n \rightarrow \infty}\left(I_{i}^{\prime} \backslash I_{i, n}^{\prime}\right) \cap\{1, \ldots, M\}=\emptyset$. Since

$$
\forall n \in \mathbb{N} \sum_{i=1, \ldots, q} \sum_{\substack{j \in I_{i, n}^{\prime} \\ j \leqslant M}} \sum_{\substack{k=1, \ldots, q \\ k \neq i}} T_{n}^{\prime}(j, k)=0
$$

sending successively $n$ and $M$ to $\infty$, we get

$$
\sum_{i=1, \ldots, q} \sum_{j \in I_{i}^{\prime}} \sum_{\substack{k=1, \ldots, q \\ k \neq i}} T^{\prime}(j, k)=0 .
$$

Let $M \in \mathbb{N}$ be fixed. For $n$ large enough, so that $\bigcup_{1 \leqslant i \leqslant q}\left(I_{i}^{\prime} \backslash I_{i, n}^{\prime}\right) \cap\{1, \ldots, M\}=\emptyset$, we have

$$
\begin{aligned}
& \operatorname{dist}_{P}\left(\left(\emptyset, \bigcup_{j \in I_{1, n}^{\prime}} A_{n}^{\prime}(j), \ldots, \bigcup_{j \in I_{q, n}^{\prime}} A_{n}^{\prime}(j)\right),\left(\emptyset, \bigcup_{j \in I_{1}^{\prime}} A^{\prime}(j), \ldots, \bigcup_{j \in I_{q}^{\prime}} A^{\prime}(j)\right)\right) \\
& \leqslant \sum_{j \leqslant M, A^{\prime}(j) \neq \emptyset} \operatorname{dist}_{L^{1}}\left(A^{\prime}(j), A_{n}^{\prime}(j)\right)+\sum_{j \leqslant M, A^{\prime}(j)=\emptyset} \mathcal{L}^{d}\left(A_{n}^{\prime}(j)\right) \\
& \quad+\sum_{j>M} 2 \mathcal{L}^{d}\left(A^{\prime}(j)\right)+\sum_{j>M} \operatorname{dist}_{L^{1}}\left(A^{\prime}(j), A_{n}^{\prime}(j)\right) .
\end{aligned}
$$

Sending successively $n$ and $M$ to $\infty$ and using the fact that $\mathbb{E}$ is closed, we see that

$$
\left(\emptyset, \bigcup_{j \in I_{1}^{\prime}} A^{\prime}(j), \ldots, \bigcup_{j \in I_{q}^{\prime}} A^{\prime}(j)\right)
$$

is in $\mathbb{E}$. Considering the partition of $\mathcal{A}^{\prime}$ in the $q$ collections $\mathcal{A}^{\prime i}=\left\{A^{\prime}(j), j \in I_{i}^{\prime}\right\}$, $1 \leqslant i \leqslant q$, we conclude that $\left(\mathcal{A}^{\prime}, \mathcal{T}^{\prime}\right)$ is still in $\mathcal{E}$.

We finally finish the proof of the upper bound in Theorem 1.3. We have

$$
\begin{aligned}
\limsup _{n \rightarrow \infty} & \frac{1}{n^{d-1}} \log \mathbb{P}_{n}\left[\left(\emptyset, D_{n}^{1}, \ldots, D_{n}^{q}\right) \in \mathbb{E}\right] \\
\leqslant & \limsup _{n \rightarrow \infty} \frac{1}{n^{d-1}} \log \Phi_{n}\left[\left(\mathcal{A}_{n}, \mathcal{T}_{n}\right) \in \mathcal{E}\right] \\
\quad & \quad \liminf _{n \rightarrow \infty} \frac{1}{n^{d-1}} \log \Phi_{n}\left[\text { no connection between } \Gamma_{n}^{1}, \ldots, \Gamma_{n}^{q}\right] .
\end{aligned}
$$

Since $\mathcal{E}$ is closed by Lemma 4.15, the large deviations upper bound of Theorem 1.8 and Lemma 4.14 yield

$$
\limsup _{n \rightarrow \infty} \frac{1}{n^{d-1}} \log \mathbb{P}_{n}\left[\left(\emptyset, D_{n}^{1}, \ldots, D_{n}^{q}\right) \in \mathbb{E}\right] \leqslant-\inf \{\mathcal{I}(\mathcal{A}, \mathcal{T}) ;(\mathcal{A}, \mathcal{T}) \in \mathcal{E}\}+\min _{P(\Omega, q)} \mathcal{I}
$$

For any $(\mathcal{A}, \mathcal{T})$ in $\mathcal{E}$, denoting by $\mathcal{A}^{1}, \ldots, \mathcal{A}^{q}$ the associated partition of $\mathcal{A}$ and by $\vec{A}$ the corresponding phase partition, that is, $\vec{A}=\left(\emptyset, \bigcup_{A \in \mathcal{A}^{1}} A, \ldots, \bigcup_{A \in \mathcal{A}^{q}} A\right)$, we have $\mathcal{I}(\mathcal{A}, \mathcal{T}) \geqslant \mathcal{I}(\vec{A})$. Therefore,

$$
-\inf \{\mathcal{I}(\mathcal{A}, \mathcal{T}) ;(\mathcal{A}, \mathcal{T}) \in \mathcal{E}\} \leqslant-\inf \{\mathcal{I}(\vec{A}) ; \vec{A} \in \mathbb{E}\}
$$

and we are done. 


\section{Appendix A}

We recall here some facts concerning the class of the sets of finite perimeter, introduced initially by Caccioppoli and subsequently developed by De Giorgi (see [19, $20,24,26,28,41,54])$. The perimeter of a Borel set $E$ of $\mathbb{R}^{d}$ is defined as

$$
\mathcal{P}(E)=\sup \left\{\int_{E} \operatorname{div} f(x) \mathrm{d} x: f \in C_{0}^{\infty}\left(\mathbb{R}^{d}, B(1)\right)\right\},
$$

where $C_{0}^{\infty}\left(\mathbb{R}^{d}, B(1)\right)$ is the set of the compactly supported $C^{\infty}$ vector functions from $\mathbb{R}^{d}$ to the unit ball $B(1)$ and div is the usual divergence operator. The set $E$ is of finite perimeter if $\mathcal{P}(E)$ is finite. A set $E$ is a Caccioppoli set if it is locally of finite perimeter. In this paper, we deal with bounded sets, hence we need only to consider sets of finite perimeter. A set $E$ has finite perimeter if and only if its characteristic function $\chi_{E}$ is a function of bounded variation. The distributional derivative $\nabla \chi_{E}$ of $\chi_{E}$ is then a vector Radon measure and $\mathcal{P}(E)=\left\|\nabla \chi_{E}\right\|\left(\mathbb{R}^{d}\right)$, where $\left\|\nabla \chi_{E}\right\|$ is the total variation measure of $\nabla \chi_{E}$. The perimeter $\mathcal{P}$ is l.s.c. on the space $\left(\mathcal{B}\left(\mathbb{R}^{d}\right)\right.$, $\left.\operatorname{dist}_{L^{1}}\right)$.

Compactness property of sets of finite perimeter. For every bounded domain $U$ and every $\lambda>0$, the set $\{E \in \mathcal{B}(U): \mathcal{P}(E) \leqslant \lambda\}$ is compact for the metric dist $L^{1}$.

This result is stated in this precise form in [20], Teorema 2.4, or [19], Teorema I. It is also an immediate consequence of the compactness theorem stated in [41], Chapter 2, p. 70. Modern presentations are formulated through functions of bounded variations: if $O$ is an open bounded domain with sufficiently regular boundary (say $C^{1}$ ), then a set of functions in $L^{1}(O)$ uniformly bounded in BV-norm is relatively compact in $L^{1}(O)$ (see any of the following references: [24], Section 5.2.3, [28], Theorem 1.19, [54], Corollary 5.3.4). To deduce the compactness result on sets of finite perimeter, we choose an open bounded domain $O$ with regular boundary containing $U$ in its interior. We embed $\mathcal{B}(U)$ in $L^{1}(O)$ by associating to a Borel set $E$ its characteristic function $\chi_{E}$ and we simply remark that the set $\left\{\chi_{E} ; E \in \mathcal{B}(U)\right\}$ is a closed subset of $L^{1}(O)$.

Let $E$ be a set of finite perimeter. Its reduced boundary $\partial^{*} E$ consists of the points $x$ such that

- $\left\|\nabla \chi_{E}\right\|(B(x, r))>0$ for any $r>0$,

- if $v_{r}(x)=-\nabla \chi_{E}(B(x, r)) /\left\|\nabla \chi_{E}\right\|(B(x, r))$ then, as $r$ goes to $0, v_{r}(x)$ converges towards a limit $v_{E}(x)$ such that $\left|v_{E}(x)\right|_{2}=1$.

The reduced boundary $\partial^{*} E$ is countably $(d-1)$-rectifiable, that is $\partial^{*} E \subset N \cup \bigcup_{i \in \mathbb{N}} M_{i}$ where $\mathcal{H}^{d-1}(N)=0$ and each $M_{i}$ is a $(d-1)$-dimensional embedded $C^{1}$ submanifold of $\mathbb{R}^{d}$. For a point $x$ belonging to $\partial^{*} E$, the vector $\nu_{E}(x)$ is called the generalized exterior normal to $E$ at $x$. A unit vector $v$ is called the measure theoretic exterior normal to $E$ at $x$ if

$$
\lim _{r \rightarrow 0} r^{-d} \mathcal{L}^{d}\left(B_{-}(x, r, v) \backslash E\right)=0, \quad \lim _{r \rightarrow 0} r^{-d} \mathcal{L}^{d}\left(B_{+}(x, r, v) \cap E\right)=0 .
$$

At each point $x$ of the reduced boundary $\partial^{*} E$ of $E$, the generalized exterior normal $v_{E}(x)$ is also the measure theoretic exterior normal to $E$ at $x$. The map $x \in \partial^{*} E \mapsto v_{E}(x) \in$ 
$S^{d-1}$ is $\left\|\nabla \chi_{E}\right\|$ measurable. For any Borel set $A$ of $\mathbb{R}^{d}$,

$$
\left\|\nabla \chi_{E}\right\|(A)=\mathcal{H}^{d-1}\left(A \cap \partial^{*} E\right), \quad \nabla \chi_{E}(A)=\int_{A \cap \partial^{*} E}-v_{E}(x) \mathcal{H}^{d-1}(\mathrm{~d} x) .
$$

We next apply the Besicovitch derivation Theorem [11] (for a quick proof, see for example [8]) to the measure $\left\|\nabla \chi_{E}\right\|$.

THEOREM A.1. - Let $f: \partial^{*} E \mapsto \mathbb{R}$ be a $\left\|\nabla \chi_{E}\right\|$ measurable bounded function. For $\mathcal{H}^{d-1}$ almost all $x$ in $\partial^{*} E$,

$$
\lim _{r \rightarrow 0}\left(\alpha_{d-1} r^{d-1}\right)^{-1} \int_{B(x, r) \cap \partial^{*} E} f(y) \mathrm{d} \mathcal{H}^{d-1}(y)=f(x) .
$$

For any vector function $f$ in $C_{0}^{1}\left(\mathbb{R}^{d}, \mathbb{R}^{d}\right)$, any Caccioppoli set $E$, by the generalized Gauss-Green Theorem,

$$
\int_{E} \operatorname{div} f(x) \mathrm{d} x=\int_{\partial^{*} E} f(x) \cdot v_{E}(x) \mathcal{H}^{d-1}(\mathrm{~d} x) .
$$

The isoperimetric inequality. There exists a positive constant $c_{\text {iso }}$ depending on the dimension only such that, for any Borel set $E$ in $\mathbb{R}^{d}$ having finite Lebesgue measure,

$$
\mathcal{L}^{d}(E) \leqslant c_{\text {iso }} \mathcal{P}(E)^{d / d-1}
$$

The Vitali covering Theorem for $\mathcal{H}^{d-1}$ (see for instance [25], Theorem 1.10). Let $E$ be a Borel subset of $\mathbb{R}^{d}$. A collection of sets $\mathcal{U}$ is called a Vitali class for $E$ if for each $x$ in $E$ and $\delta$ positive there exists a set $U$ in $\mathcal{U}$ containing $x$ such that $0<\operatorname{diam}_{2} U<\delta$.

THEOREM A.2.- Let $E$ be an $\mathcal{H}^{d-1}$-measurable subset of $\mathbb{R}^{d}$ and let $\mathcal{U}$ be a Vitali class of closed sets for $E$. Then we may select a (countable) disjoint sequence $\left(U_{i}\right)_{i \in I}$ from $\mathcal{U}$ such that either $\sum_{i \in I}\left(\operatorname{diam} U_{i}\right)^{d-1}=\infty$ or $\mathcal{H}^{d-1}\left(E \backslash \bigcup_{i \in I} U_{i}\right)=$ 0 . If $\mathcal{H}^{d-1}(E)<\infty$ then, given $\varepsilon>0$, we may also require that $\mathcal{H}^{d-1}(E) \leqslant$ $\alpha_{d-1} 2^{-d+1} \sum_{i \in I}\left(\operatorname{diam}_{2} U_{i}\right)^{d-1}+\varepsilon$.

The Minkowski content (see [26], 3.2.36). The $(d-1)$-dimensional Minkowski content of a subset $E$ of $\mathbb{R}^{d}$ is equal to the limit, if it exists,

$$
\lim _{r \rightarrow 0} \frac{1}{2 r} \mathcal{L}^{d}\left(\left\{x \in \mathbb{R}^{d}: d_{2}(x, E)<r\right\}\right) .
$$

Whenever $E$ is $(d-1)$-rectifiable (i.e. there exists a Lipschitz function mapping some bounded subset of $\mathbb{R}^{d-1}$ onto $E$ ) and closed, the Minkowski content of $E$ is equal to $\mathcal{H}^{d-1}(E)$ (see [26], Theorem 3.2.39).

THEOREM A.3 (Strong approximation of sets of finite perimeter [50]). - Let $O$ be an open set in $\mathbb{R}^{d}(d \geqslant 2)$. Let $E \subset O$ be a set of finite perimeter in $O$ and let $\varepsilon$ be positive. There exists a set $L \subseteq O$ of finite perimeter in $O$ such that: 
(a) $O \cap \partial L$ is included in a finite union of $C^{1}$ hypersurfaces,

(b) $L \subseteq \mathcal{V}_{2}(E, \varepsilon), O \backslash L \subseteq \mathcal{V}_{2}(O \backslash E, \varepsilon)$,

(c) $\mathcal{L}^{d}(O \cap(E \Delta L))<\varepsilon, \mathcal{H}^{d-1}\left(O \cap\left(\partial^{*} E \Delta \partial L\right)\right)<\varepsilon$.

More precisely, there exists a compact set $C$ in $O, a C^{1}$ function $f: \mathbb{R}^{d} \mapsto \mathbb{R}$ and open sets $V, B$ and $G$ in $O$ such that, setting $F=\{x \in O: f(x) \geqslant 0\}$ :

$$
\begin{aligned}
& C \subset O \cap \partial^{*} E, \quad \mathcal{H}^{d-1}\left(O \cap \partial^{*} E \backslash C\right)<\varepsilon, \\
& \forall x \in C \quad f(x)=0, \quad v_{E}(x)=v_{F}(x)=-\mathrm{d} f(x), \\
& C \subset V \subset\{x \in O: \mathrm{d} f(x) \neq 0\}, \\
& V \cap \partial^{*} F=V \cap \partial F=\{x \in V: f(x)=0\}, \text { which is a } C^{1} \text { hypersurface, } \\
& \mathcal{L}^{d}(V)<\varepsilon, \quad \mathcal{H}^{d-1}(\partial F \cap(V \backslash C))<\varepsilon, \\
& C \subset B \subset V, B \text { is a finite union of open cubes, } \\
& O \cap \partial G \text { is a } C^{\infty} \text { hypersurface, }
\end{aligned}
$$$$
\text { the set } L=(F \cap \bar{B}) \cup(G \cap O \backslash B) \text { satisfies (a), (b), (c). }
$$

LEMMA A.4. - Let $\Gamma$ be an hypersurface (that is a $C^{1}$ submanifold of $\mathbb{R}^{d}$ of codimension 1) and let $K$ be a compact subset of $\Gamma$. There exists a positive $M=$ $M(\Gamma, K)$ such that:

$$
\forall \varepsilon>0 \quad \exists r>0 \quad \forall x, y \in K \quad|x-y|_{2} \leqslant r \Rightarrow d_{2}(y, \tan (\Gamma, x)) \leqslant M \varepsilon|x-y|_{2}
$$

$(\tan (\Gamma, x)$ is the tangent hyperplane of $\Gamma$ at $x)$.

Proof. - By a standard compactness argument, it is enough to prove the following local property:

$$
\begin{aligned}
& \forall x \in \Gamma \quad \exists M(x)>0 \quad \forall \varepsilon>0 \quad \exists r(x, \varepsilon)>0 \quad \forall y, z \in \Gamma \cap B(x, r(x, \varepsilon)) \\
& d_{2}(y, \tan (\Gamma, z)) \leqslant M(x) \varepsilon|y-z|_{2} .
\end{aligned}
$$

Indeed, if this property holds, we cover $K$ by the open balls $\stackrel{\circ}{B}(x, r(x, \varepsilon) / 2), x \in K$, we extract a finite subcovering $\stackrel{\circ}{B}\left(x_{i}, r\left(x_{i}, \varepsilon\right) / 2\right), 1 \leqslant i \leqslant k$, and we set

$$
M=\max \left\{M\left(x_{i}\right): 1 \leqslant i \leqslant k\right\}, \quad r=\min \left\{r\left(x_{i}, \varepsilon\right) / 2: 1 \leqslant i \leqslant k\right\} .
$$

Let now $y, z$ belong to $K$ with $|y-z|_{2} \leqslant r$. Let $i$ be such that $y$ belongs to $B\left(x_{i}, r\left(x_{i}, \varepsilon\right) / 2\right)$. Since $r \leqslant r\left(x_{i}, \varepsilon\right) / 2$, then both $y, z$ belong to the ball $B\left(x_{i}, r\left(x_{i}, \varepsilon\right)\right)$ and it follows that $d_{2}(y, \tan (\Gamma, z)) \leqslant M\left(x_{i}\right) \varepsilon|y-z|_{2} \leqslant M \varepsilon|y-z|_{2}$.

We turn now to the proof of the above local property. Since $\Gamma$ is an hypersurface, for any $x$ in $\Gamma$ there exists a neighborhood $V$ of $x$ in $\mathbb{R}^{d}$, a diffeomorphism $f: V \mapsto \mathbb{R}^{d}$ of class $C^{1}$ and a $(d-1)$-dimensional vector space $Z$ of $\mathbb{R}^{d}$ such that $Z \cap f(V)=f(\Gamma \cap V)$ (see for instance [26], 3.1.19). Let $A$ be a compact neighborhood of $x$ included in $V$. Since $f$ is a diffeomorphism, the maps $y \in A \mapsto \mathrm{d} f(y) \in \operatorname{End}\left(\mathbb{R}^{d}\right), u \in f(A) \mapsto$ $\mathrm{d} f^{-1}(u) \in \operatorname{End}\left(\mathbb{R}^{d}\right)$ are continuous. Therefore they are bounded:

$$
\exists M>0 \quad \forall y \in A \quad\|\mathrm{~d} f(y)\| \leqslant M, \quad \forall u \in f(A) \quad\left\|\mathrm{d} f^{-1}(u)\right\| \leqslant M
$$


(here $\|\mathrm{d} f(x)\|=\sup \left\{|\mathrm{d} f(x)(y)|_{2}:|y|_{2} \leqslant 1\right\}$ is the standard operator norm in $\operatorname{End}\left(\mathbb{R}^{d}\right)$ ). Since $f(A)$ is compact, the differential map $\mathrm{d} f^{-1}$ is uniformly continuous on $f(A)$ :

$$
\forall \varepsilon>0 \quad \exists \delta>0 \quad \forall u, v \in f(A) \quad|u-v|_{2} \leqslant \delta \Rightarrow\left\|\mathrm{d} f^{-1}(u)-\mathrm{d} f^{-1}(v)\right\| \leqslant \varepsilon .
$$

Let $\varepsilon$ be positive and let $\delta$ be associated to $\varepsilon$ as above. Let $\rho$ be positive and small enough so that $\rho<\delta / 2$ and $B(f(x), \rho) \subset f(A)$ (since $f$ is a $C^{1}$ diffeomorphism, $f(A)$ is a neighborhood of $f(x))$. Let $r$ be such that $0<r<\rho / M$ and $B(x, r) \subset A$. We claim that $M$ associated to $x$ and $r$ associated to $\varepsilon, x$ answer the problem. Let $y, z$ belong to $\Gamma \cap B(x, r)$. Since $[y, z] \subset B(x, r) \subset A$, and $\|\mathrm{d} f(\zeta)\| \leqslant M$ on $A$, then

$$
\begin{aligned}
& |f(y)-f(x)|_{2} \leqslant M|y-x|_{2} \leqslant M r<\rho, \quad|f(z)-f(x)|_{2}<\rho, \\
& |f(y)-f(z)|_{2}<\delta, \quad|f(y)-f(z)|_{2}<M|y-z|_{2} .
\end{aligned}
$$

We apply next a classical lemma of differential calculus (see [37], I, 4, Corollary 2) to the map $f^{-1}$ and the interval $[f(z), f(y)]$ (which is included in $B(f(x), \rho) \subset f(A)$ ) and the point $f(z)$ :

$$
\begin{aligned}
& \left|y-z-\mathrm{d} f^{-1}(f(z))(f(y)-f(z))\right|_{2} \\
& \quad \leqslant|f(y)-f(z)|_{2} \sup \left\{\left\|\mathrm{d} f^{-1}(\zeta)-\mathrm{d} f^{-1}(f(z))\right\|: \zeta \in[f(z), f(y)]\right\} .
\end{aligned}
$$

The right-hand member is less than $M|y-z|_{2} \varepsilon$. Since $z+\mathrm{d} f^{-1}(f(z))(f(y)-f(z))$ belongs to $\tan (\Gamma, z)$, we are done.

\section{Acknowledgement}

The authors thank Patrice Assouad and Thierry Quentin de Gromard for their careful reading of the section dealing with geometric measure theory and their numerous comments.

\section{REFERENCES}

[1] Alexander K.S., Cube-root boundary fluctuations for droplets in random cluster models, Preprint, 2000.

[2] Almgren F.J. Jr., Existence and regularity almost everywhere of solutions to elliptic variational problems with constraints, Mem. Amer. Math. Soc. 4 (165) (1976).

[3] Alberti G., Bellettini G., Cassandro M., Presutti E., Surface tension in Ising systems with Kac potentials, J. Stat. Phys. 82 (1996) 743-796.

[4] Alexander K.S., Chayes J.T., Chayes L., The Wulff construction and asymptotics of the finite cluster distribution for two-dimensional Bernoulli percolation, Comm. Math. Phys. 131 (1990) 1-50.

[5] Ambrosio L., Braides A., Functionals defined on partitions in sets of finite perimeter I: Integral representation and $\Gamma$-convergence, J. Math. Pures et Appl. 69 (1990) 285-305.

[6] Ambrosio L., Braides A., Functionals defined on partitions in sets of finite perimeter II: Semicontinuity, relaxation and homogenization, J. Math. Pures et Appl. 69 (1990) 307333.

[7] Ambrosio L., Novaga M., Paolini E., Some regularity results for minimal crystals, Preprint, 2000. 
[8] Assouad P., Quentin de Gromard T., Sur la dérivation des mesures dans $\mathbb{R}^{n}$, Note (1998).

[9] Benois O., Bodineau T., Buttà P., Presutti E., On the validity of van der Waals theory of surface tension, Markov Process. Rel. Fields 3 (1997) 175-198.

[10] Benois O., Bodineau T., Presutti E., Large deviations in the van der Waals limit, Stochastic Process. Appl. 75 (1998) 89-104.

[11] Besicovitch A.S., A general form of the covering principle and relative differentiation of additive functions, Proc. Cambridge Philos. Soc. 41 (1945) 103-110. Part II. Proc. Cambridge Philos. Soc. 42 (1946) 1-10.

[12] Bodineau T., The Wulff construction in three and more dimensions, Comm. Math. Phys. 207 (1) (1999) 197-229.

[13] Bodineau T., Ioffe D., Velenik Y., Rigorous probabilistic analysis of equilibrium crystal shapes, J. Math. Phys. 41 (3) (2000) 1033-1098.

[14] Cerf R., Large deviations for three dimensional supercritical percolation, Astérisque 267 (2000).

[15] Cerf R., Pisztora A., On the Wulff crystal in the Ising model, Ann. Probab. 28 (3) (2000) 945-1015.

[16] Congedo G., Tamanini I., Optimal partitions with unbounded data, Atti Accad. Naz. Lincei, Cl. Sci. Fis. Mat. Nat., IX Ser., Rend. Lincei., Mat. Appl. 4 (2) (1993) 103-108.

[17] Congedo G., Tamanini I., On the existence of solutions to a problem in multidimensional segmentation, Ann. Inst. Henri Poincaré, Anal. Non Linéaire 8 (2) (1991) 175-195.

[18] Congedo G., Tamanini I., Regularity properties of optimal segmentations, J. Reine Angew. Math. 420 (1991) 61-84.

[19] De Giorgi E., Nuovi teoremi relativi alle misure $(r-1)$-dimensionali in uno spazio ad $r$ dimensioni, Ricerche Mat. 4 (1955) 95-113.

[20] De Giorgi E., Colombini F., Piccinini L.C., Frontiere orientate di misura minima e questioni collegate, Scuola Normale Superiore di Pisa (1972).

[21] Deuschel J.-D., Pisztora A., Surface order large deviations for high-density percolation, Probab. Theory Relat. Fields 104 (1996) 467-482.

[22] Dobrushin R.L., Kotecký R., Shlosman S.B., Wulff Construction: A Global Shape from Local Interaction, AMS Translations Series, Providence, RI, 1992.

[23] Dobrushin R.L., Shlosman S.B., Thermodynamic inequalities for the surface tension and the geometry of the Wulff construction, in: Albeverio S. (Ed.), Ideas and Methods in Quantum and Statistical Physics, Cambridge University Press, 1992, pp. 461-483.

[24] Evans L.C., Gariepy R.F., Measure Theory and Fine Properties of Functions, Studies in Advanced Mathematics, CRC Press, Boca Raton, 1992.

[25] Falconer K.J., The Geometry of Fractal Sets, Cambridge Tracts in Mathematics, Vol. 85, Cambridge Univ. Press, 1985.

[26] Federer H., Geometric Measure Theory, Springer-Verlag, 1969.

[27] Fortuin C.M., Kasteleyn P.W., On the random-cluster model. I. Introduction and relation to other models, Physica 57 (1972) 536-564.

[28] Giusti E., Minimal Surfaces and Functions of Bounded Variation, Birkhäuser, 1984.

[29] Grimmett G.R., Percolation, 2nd edn., Grundlehren der Mathematischen Wissenschaften, Vol. 321, Springer-Verlag, Berlin, 1999.

[30] Grimmett G.R., The stochastic random-cluster process and the uniqueness of randomcluster measures, Ann. Probab. 23 (1995) 1461-1510.

[31] Grimmett G.R., Marstrand J.M., The supercritical phase of percolation is well behaved, Proc. R. Soc. Lond. Ser. A 430 (1990) 439-457.

[32] Hass J., Hutchings M., Schlafly R., The double bubble conjecture, Electron. Res. Announc. Amer. Math. Soc. 1 (3) (1995) 98-102 (electronic). 
[33] Ioffe D., Large deviations for the 2D Ising model: a lower bound without cluster expansions, J. Stat. Phys. 74 (1993) 411-432.

[34] Ioffe D., Exact large deviation bounds up to $T_{c}$ for the Ising model in two dimensions, Probab. Theory Relat. Fields 102 (1995) 313-330.

[35] Ioffe D., Schonmann R., Dobrushin-Kotecký-Shlosman Theorem up to the critical temperature, Comm. Math. Phys. 199 (1998) 117-167.

[36] Kesten H., Zhang Y., The probability of a large finite cluster in supercritical Bernoulli percolation, Ann. Probab. 18 (1990) 537-555.

[37] Lang S., Differential Manifolds, 2nd edn., Springer-Verlag, 1985.

[38] Lawlor G., Morgan F., Paired calibrations applied to soap films, immiscible fluids, and surfaces or networks minimizing other norms, Pacific J. Math. 166 (1) (1994) 55-82.

[39] Lebowitz J.L., Mazel A.E., Suhov Yu.M., An Ising interface between two walls: competition between two tendencies, Rev. Math. Phys. 8 (5) (1996) 669-687.

[40] Leonardi G.P., Optimal subdivisions of $n$-dimensional domains. Ph.D. Thesis, Università di Trento, 1998.

[41] Massari U., Miranda M., Minimal Surfaces of Codimension One, North-Holland Mathematics Studies 91, Notas de Matematica, Vol. 95, North-Holland, 1984.

[42] Massari U., Pepe L., Sull'approssimazione degli aperti lipschitziani di $\mathbb{R}^{n}$ con varietà differenziabili, Bollettino U.M.I. (4) 10 (1974) 532-544.

[43] Messager A., Miracle-Solé S., Ruiz J., Convexity properties of the surface tension and equilibrium crystals, J. Stat. Phys. 67 (3/4) (1992) 449-469.

[44] Miracle-Solé S., Surface tension, step free energy, and facets in the equilibrium crystal, J. Stat. Phys. 79 (1/2) (1995) 183-214.

[45] Newman C.M., Topics in Disordered Systems, Lectures in Mathematics, ETH Zürich, Birkhäuser, 1997.

[46] Pfister C.E., Large deviations and phase separation in the two-dimensional Ising model, Helv. Phys. Acta 64 (1991) 953-1054.

[47] Pfister C.E., Velenik Y., Interface, surface tension and reentrant pinning transition in the 2D Ising model, Comm. Math. Phys. 204 (2) (1999) 269-312.

[48] Pfister C.E., Velenik Y., Large deviations and continuum limit in the 2D Ising model, Probab. Theory Related Fields 109 (1997) 435-506.

[49] Pisztora A., Surface order large deviations for Ising, Potts and percolation models, Probab. Theory Related Fields 104 (1996) 427-466.

[50] Quentin de Gromard T., Approximation forte dans $B V(\Omega)$, C. R. Acad. Sci. Paris, Ser. I 301 (1985) 261-264.

[51] Schonmann R.H., Second order large deviation estimates for ferromagnetic systems in the phase coexistence region, Comm. Math. Phys. 112 (1987) 409-422.

[52] Velenik Y., Ph.D. Thesis, EPFL, 1997.

[53] Vol'pert A.I., The spaces BV and quasilinear equations, Math. USSR Sb. 2 (1967) 225-267.

[54] Ziemer W.P., Weakly Differentiable Functions. Sobolev Spaces and Functions of Bounded Variation, Graduate Texts in Mathematics, Vol. 120, Springer-Verlag, 1989. 